REPRESENTATION THEORY

An Electronic Journal of the American Mathematical Society

Volume 15, Pages 494-530 (June 8, 2011)

S 1088-4165(2011)00396-4

\title{
FROM CONJUGACY CLASSES IN THE WEYL GROUP TO UNIPOTENT CLASSES
}

\author{
G. LUSZTIG
}

\begin{abstract}
Let $G$ be a connected reductive algebraic group over an algebraic closed field. We define a (surjective) map from the set of conjugacy classes in the Weyl group to the set of unipotent classes in $G$.
\end{abstract}

\section{INTRODUCTION}

0.1. Let $G$ be a connected reductive algebraic group over an algebraically closed field $\mathbf{k}$ of characteristic $p \geq 0$. Let $\underline{G}$ be the set of conjugacy classes in $G$. Let $\underline{G}$ be the set of unipotent conjugacy classes in $G$. Let $\underline{\mathbf{W}}$ be the set of conjugacy classes in the Weyl group $\mathbf{W}$ of $G$. In $[\mathrm{KL}$ a (conjecturally injective) map $\underline{G} \rightarrow \underline{\mathbf{W}}$ was defined, assuming that $\mathbf{k}=\mathbf{C}$; the definition in $\mathrm{KL}$ was in terms of the Lie algebra of $G$ with scalars extended to the power series field $\mathbf{C}((\epsilon))$. (The idea that a relationship between $\underline{\underline{G}}$ and $\underline{\mathbf{W}}$ might exist appeared in Carter's paper Ca. .) In this paper, developing an idea in $\underline{\mathrm{L} 6}$, we define a surjective map $\Phi: \underline{\mathbf{W}} \rightarrow \underline{G}$. Our definition of $\Phi$ is not in terms of the Lie algebra but in terms of the group and it works in any characteristic (but for the purposes of this introduction we assume that $p$ is not a bad prime for $G$ ). More precisely, we look at the intersection of a Bruhat double coset of $G$ with various unipotent conjugacy classes and we select the minimal unipotent class which gives a nonempty intersection. (We assume that the Bruhat double coset corresponds to a Weyl group element which has minimal length in its conjugacy class.) The fact that such a procedure might work is suggested by the statement in Steinberg [St, 8.8] that the Bruhat double coset corresponding to a Coxeter element of minimal length intersects exactly one unipotent class (the regular one), by the result in Kawanaka $\mathrm{Ka}$ that the regular unipotent class of $G$ intersects every Bruhat double coset, and by the examples in rank $\leq 3$ given in [L6. But the fact that the procedure actually works is miraculous. In this paper the proof is given separately for classical groups; for exceptional groups the desired result is reduced, using the representation theory of reductive groups over a finite field, to a computer calculation; see $§ 1.2$. I thank Gongqin Li for doing the programming involved in the calculation.

0.2. Here is some notation that we use in this paper. Let $Z_{G}$ be the centre of $G$. Let $\mathcal{B}$ be the variety of Borel subgroups of $G$. Let $\mathbf{W}$ be a set indexing the set of orbits of $G$ acting on $\mathcal{B} \times \mathcal{B}$ by $g:\left(B, B^{\prime}\right) \mapsto\left(g B g^{-1}, g B^{\prime} g^{-1}\right)$. For $w \in \mathbf{W}$ we write $\mathcal{O}_{w}$ for the corresponding $G$-orbit in $\mathcal{B} \times \mathcal{B}$. Define $\underline{l}: \mathbf{W} \rightarrow \mathbf{N}$ by

Received by the editors April 22, 2010 and, in revised form, August 11, 2010.

2010 Mathematics Subject Classification. Primary 20G99.

Supported in part by the National Science Foundation.

(C)2011 American Mathematical Society Reverts to public domain 28 years from publication 
$\underline{l}(w)=\operatorname{dim} \mathcal{O}_{w}-\operatorname{dim} \mathcal{B}$. Let $S=\{s \in \mathbf{W}: \underline{l}(s)=1\}$. There is a unique group structure on $\mathbf{W}$ such that $s^{2}=1$ for all $s \in S$ and such that

$$
\begin{aligned}
& w \in \mathbf{W}, w^{\prime} \in \mathbf{W},\left(B_{1}, B_{2}\right) \in \mathcal{O}_{w},\left(B_{2}, B_{3}\right) \in \mathcal{O}_{w^{\prime}}, \underline{l}\left(w w^{\prime}\right)=\underline{l}(w)+\underline{l}\left(w^{\prime}\right) \Longrightarrow \\
& \left(B_{1}, B_{3}\right) \in \mathcal{O}_{w w^{\prime}} .
\end{aligned}
$$

Then $\mathbf{W}, S$ is a finite Coxeter group with length function $\underline{l}$ (the Weyl group of $G$ ). Let $\underline{\mathbf{W}}$ be the set of conjugacy classes in $\mathbf{W}$. For any $C \in \underline{\mathbf{W}}$, let $d_{C}=\min _{w \in C} \underline{l}(w)$ and let $C_{\min }=\left\{w \in C: \underline{l}(w)=d_{C}\right\}$. For any $w \in \mathbf{W}$, let

$$
\mathfrak{B}_{w}=\left\{(g, B) \in G \times \mathcal{B}:\left(B, g B g^{-1}\right) \in \mathcal{O}_{w}\right\} .
$$

(This variety enters in an essential way in the definition of character sheaves on $G$.) We have a partition $\mathfrak{B}_{w}=\bigsqcup_{\gamma \in \underline{G}} \mathfrak{B}_{w}^{\gamma}$, where

$$
\mathfrak{B}_{w}^{\gamma}=\left\{(g, B) \in \mathfrak{B}_{w}: g \in \gamma\right\} .
$$

Note that $G$ and $G_{a d}:=G / Z_{G}$ act on $\mathfrak{B}_{w}$ and on $\mathfrak{B}_{w}^{\gamma}$ (for $\gamma \in \underline{G}$ ) by $x:(g, B) \mapsto$ $\left(x g x^{-1}, x B x^{-1}\right), x Z_{G}:(g, B) \mapsto\left(x g x^{-1}, x B x^{-1}\right)$. For $\gamma \in \underline{G}, C \in \underline{\mathbf{W}}$ we write $C \dashv \gamma$ if $\mathfrak{B}_{w}^{\gamma} \neq \emptyset$ for some/any $w \in C_{\min }$. (The equivalence of some/any follows from 1.2(a), using [GP, 8.2.6(b)].) For $\gamma \in \underline{G}$ we denote by $\bar{\gamma}$ the closure of $\gamma$ in $G$.

For any $J \subset S$, let $\mathbf{W}_{J}$ be the subgroup of $\mathbf{W}$ generated by $J$. We say that $C \in \underline{\mathbf{W}}$ is elliptic if $C \cap \mathbf{W}_{J}=\emptyset$ for any $J \varsubsetneqq S$. Let

$$
\underline{\mathbf{W}}_{e l}=\{C \in \underline{\mathbf{W}}: C \text { elliptic }\} .
$$

If $P$ is a parabolic subgroup of $G$ there is a unique subset $J \subset S$ (said to be the type of $P$ ) such that

$$
\left\{w \in \mathbf{W}:\left(B, B^{\prime}\right) \in \mathcal{O}_{w} \text { for some } B \subset P, B^{\prime} \subset P\right\}=\mathbf{W}_{J} .
$$

For an integer $\sigma$ we define $\kappa_{\sigma} \in\{0,1\}$ by $\sigma=\kappa_{\sigma} \bmod 2$. For two integers $a, b$ we set $[a, b]=\{c \in \mathbf{Z}: a \leq c \leq b\}$. The cardinality of a finite set $X$ is denoted by $|X|$ or by $\sharp(X)$. For $g \in G, Z(g)$ denotes the centralizer of $g$ in $G$. Let $C_{c o x}$ be the conjugacy class in $\mathbf{W}$ that contains the Coxeter elements. For any parabolic subgroup $P$ of $G$ let $U_{P}$ be the unipotent radical of $P$.

0.3. Let $C \in \underline{\mathbf{W}}$. Consider the following property:

$\Pi_{C}$. There exists $\gamma \in \underline{\underline{G}}$ such that $C \dashv \gamma$ and such that if $\gamma^{\prime} \in \underline{\underline{G}}$ and $C \dashv \gamma^{\prime}$, then $\gamma \subset \bar{\gamma}^{\prime}$.

Note that if $\Pi_{C}$ holds, then $\gamma$ is uniquely determined; we denote it by $\gamma_{C}$.

We state our main result.

Theorem 0.4. Assume that $p$ is not a bad prime for $G$. Then

(i) $\Pi_{C}$ holds for any $C \in \underline{\mathbf{W}}$;

(ii) the map $\underline{\mathbf{W}} \rightarrow \underline{\underline{G}}, C \mapsto \gamma_{C}$ is surjective.

0.5. Recall that $\gamma \in \underline{\underline{G}}$ is distinguished if for some/any $g \in G, g$ is not contained in a Levi subgroup of a proper parabolic subgroup of $G$. In $\S 1.1$ it is shown how Theorem 0.4 can be deduced from the following result.

Proposition 0.6. Assume that $p$ is not a bad prime for $G$. Then

(i) $\Pi_{C}$ holds for any $C \in \underline{\mathbf{W}}_{e l}$;

(ii) the map $\underline{\mathbf{W}}_{e l} \rightarrow \underline{\underline{G}}, C \mapsto \gamma_{C}$ is injective and its image contains all distinguished unipotent classes of $G$. 
The following result provides an alternative definition for the the map in Proposition 0.6(ii).

Theorem 0.7. Assume that $p$ is not a bad prime for $G$. Let $C \in \underline{\mathbf{W}}_{e l}$. Let $w \in C_{\min }$.

(a) If $\gamma \in \underline{G}$ and $\mathfrak{B}_{w}^{\gamma} \neq \emptyset$, then $\operatorname{dim} Z(g) / Z_{G} \leq d_{C}$ for some/all $g \in \gamma$.

(b) There is a unique unipotent class $\gamma$ in $G$ such that $\mathfrak{B}_{w}^{\gamma} \neq \emptyset$ and $\operatorname{dim} Z(g) / Z_{G}$ $=d_{C}$ for some/all $g \in \gamma$.

(c) The class $\gamma$ in (b) depends only on $C$, not on $w$. It coincides with $\gamma_{C}$ in Proposition 0.6(ii).

This follows from results in $\S 5$.

0.8. This paper is organized as follows. Section 1 contains some preparatory material. In Section 2 we define a particular class of reduced decompositions for certain elliptic elements of $\mathbf{W}$. To such a decomposition we attach a unipotent element in $G$. We study this element in several cases arising from classical groups. This provides one of the ingredients in the proof of Proposition 0.6 for classical groups. (It might also provide an alternative definition for our map $\underline{\mathbf{W}} \rightarrow \underline{G}$; see the conjecture in $\S 4.7$.) In Section 3 we complete the proof of Proposition 0.6 for classical groups. In Section 4 we extend our results to arbitrary characteristic. In $\S 4.3$ we give an explicit description of the restriction of the map $\Phi: \underline{\mathbf{W}} \rightarrow \underline{G}$ to $\underline{\mathbf{W}}_{e l}$ for various almost simple $G$. In $\S 5$ we associate to any $C \in \underline{\mathbf{W}}_{e l}$ a collection of conjugacy classes in $G$, said to be $C$-small classes: the conjugacy classes $\gamma \in \underline{G}$ of minimum dimension such that $C \dashv \gamma$; we also verify Theorem 0.7 .

0.8. For earlier work on the intersection of Bruhat double cosets with conjugacy classes in $G$, see EG. (I thank Jiang-Hua Lu for this reference.)

\section{Preliminaries}

1.1. We show how Theorem 0.4 can be proved assuming that Proposition 0.6 holds when $G$ is replaced by any Levi subgroup of a parabolic subgroup of $G$. Let $C \in \underline{\mathbf{W}}$. If $C \in \underline{\mathbf{W}}_{e l}$, then the result follows from our assumption. We now assume that $C$ is not elliptic. We can find $J \varsubsetneqq S$ and an elliptic conjugacy class $D$ of the Weyl group $\mathbf{W}_{J}$ such that $D=C \cap \mathbf{W}_{J}$. Let $P$ be a parabolic subgroup of $G$ of type $J$. Let $L$ be a Levi subgroup of $P$. Let $\gamma_{D}$ be the unipotent class of $L$ associated to $D$ by Proposition 0.6(i) with $G, \mathbf{W}$ replaced by $L, \mathbf{W}_{J}$. Let $\gamma$ be the unipotent class of $G$ containing $\gamma_{D}$. Let $g \in \gamma, w \in D$. Note that some $G$-conjugate $g^{\prime}$ of $g$ is contained in $L$. We can find Borel subgroups $B, B^{\prime}$ of $P$ such that $\left(B, B^{\prime}\right) \in \mathcal{O}_{w}, B^{\prime}=g^{\prime} B g^{\prime-1}$. Thus $\mathfrak{B}_{w}^{\gamma} \neq \emptyset$. Now let $\gamma^{\prime}$ be a unipotent class of $G$ such that $\mathfrak{B}_{w^{\prime}}^{\gamma^{\prime}} \neq \emptyset$ for some $w^{\prime} \in C_{\min }$. We have $C_{\min } \cap D \neq \emptyset$ (see [GP, 3.1.14]); hence we can assume that $w^{\prime} \in D$. We can find $\left(B, B^{\prime}\right) \in \mathcal{O}_{w^{\prime}}$ and $g^{\prime} \in \gamma^{\prime}$ such that $B^{\prime}=g^{\prime} B g^{\prime-1}$. Replacing $B, B^{\prime}, g^{\prime}$ by $x B x^{-1}, x B^{\prime} x^{-1}, x g^{\prime} x^{-1}$ for some $x \in G$ we see that we can assume that $B \subset P$ and then we automatically have $B^{\prime} \subset P$, that is, $g^{\prime} B g^{\prime-1} \subset P$. We have also $g^{\prime} B g^{-1} \subset g^{\prime} P g^{\prime-1}$; hence $g^{\prime} P g^{-1}=P$, that is, $g^{\prime} \in P$. We have $g^{\prime}=g_{1}^{\prime} v$, where $g_{1}^{\prime} \in L$ is unipotent and $v \in U_{P}$. We can find a one-parameter subgroup $\lambda: \mathbf{k}^{*} \rightarrow Z_{L}$ such that $\lambda(t) v \lambda\left(t^{-1}\right)$ converges to 1 when $t \in \mathbf{k}^{*}$ converges to 0 . Then $\lambda(t) g^{\prime} \lambda(t)^{-1}=g_{1}^{\prime} \lambda(t) v \lambda(t)^{-1}$ converges to $g_{1}^{\prime}$ when $t \in \mathbf{k}^{*}$ converges to 0 . Thus $g_{1}^{\prime}$ is contained in the closure of $\gamma^{\prime}$. Hence the $L$-conjugacy class of $g_{1}^{\prime}$ is contained in the closure of $\gamma^{\prime}$. Note also that $B^{\prime}=g_{1}^{\prime} B g_{1}^{\prime-1}$. Using the definition of $\gamma_{D}$ 
we see that $\gamma_{D}$ is contained in the closure of the $L$-conjugacy class of $g_{1}^{\prime}$. Hence $\gamma_{D}$ is contained in the closure of $\gamma^{\prime}$ and $\gamma$ is contained in the closure of $\gamma^{\prime}$. We see that $\gamma$ has the property stated in $\Pi_{C}$. This proves Theorem $0.4(\mathrm{i})$ (assuming Proposition 0.6(i)).

The previous argument shows that $\gamma_{C}$ is the unipotent class of $G$ containing the unipotent class $\gamma_{D}$ of $L$. Thus $C \mapsto \gamma_{C}$ is determined in a simple way from the knowledge of the maps $D \mapsto \gamma_{D}$ in Proposition 0.6 corresponding to various $L$ as above.

Now let $\gamma \in G$. We can find a parabolic subgroup $P$ of $G$ with Levi subgroup $L$ and a distinguished unipotent class $\gamma_{1}$ of $L$ such that $\gamma_{1} \subset \gamma$. Let $J$ be the subset $S$ such that $P$ is of type $J$. By Proposition 0.6(ii) we can find an elliptic conjugacy class $D$ of $\mathbf{W}_{J}$ such that $\gamma_{D}=\gamma_{1}$ (where $\gamma_{D}$ is defined in terms of $L, D$ ). Let $C$ be the conjugacy class in $\mathbf{W}$ that contains $D$. By the arguments above we have $\gamma_{C}=\gamma$. This proves Theorem 0.4(ii) (assuming Proposition 0.6).

1.2. To prove Proposition 0.6 we can assume that $G$ is almost simple. Moreover for each isogeny class of almost simple groups it is enough to prove Proposition 0.6 for one group in the isogeny class and then Proposition 0.6 will be automatically true for the other groups in the isogeny class.

Note that if $C=C_{c o x}$ (recall that $C \in \underline{\mathbf{W}}_{e l}$ ), then $\Pi_{C}$ follows from a statement in [St, 8.8]; in this case $\gamma_{C}$ is the regular unipotent class. If $G$ is almost simple of type $A_{n}$, then $C$ as above is the only element of $\underline{\mathbf{W}}_{e l}$ and the only distinguished unipotent class is the regular one so that in this case Proposition 0.6 follows.

If $G$ is almost simple of type $B_{n}, C_{n}$ or $D_{n}$, then we can assume that $G$ is as in $\S 1.3$. The proof of Proposition 0.6 in these cases is given in $\S \S 3.7-3.9$.

In the remainder of this subsection we assume that $\mathbf{k}$ is an algebraic closure of a finite field $\mathbf{F}_{q}$ with $q$ elements. We choose an $\mathbf{F}_{q}$-split rational structure on $G$ with Frobenius map $F: G \rightarrow G$. Now $F$ induces a morphism $\mathcal{B} \rightarrow \mathcal{B}$ denoted again by $F$. Note that the finite group $G^{F}$ acts transitively on the finite set $\mathcal{B}^{F}$ (the upper script denotes the set of fixed points). Hence $G^{F}$ acts naturally on the $\overline{\mathbf{Q}}_{l}$-vector space $\mathcal{F}$ of functions $\mathcal{B}^{F} \rightarrow \overline{\mathbf{Q}}_{l}$. (Here $l$ is a fixed prime number such that $l \neq 0$ in k.) For any $w \in \mathbf{W}$ we denote by $T_{w}: \mathcal{F} \rightarrow \mathcal{F}$ the linear map $f \mapsto f^{\prime}$, where $f^{\prime}(B)=\sum_{B^{\prime} \in \mathcal{B}^{F} ;\left(B, B^{\prime}\right) \in \mathcal{O}_{w}} f\left(B^{\prime}\right)$. Let $\mathcal{H}_{q}$ be the subspace of $\operatorname{End}(\mathcal{F})$ spanned by $T_{w}(w \in \mathbf{W})$; this is a subalgebra of $\operatorname{End}(\mathcal{F})$ and the irreducible $\mathcal{H}_{q}$-modules (up to isomorphism) are in natural bijection $E_{q} \leftrightarrow E$ with $\operatorname{Irr} \mathbf{W}$, the set of irreducible $\mathbf{W}$ modules over $\overline{\mathbf{Q}}_{l}$ (up to isomorphism) once $\sqrt{q}$ has been chosen. Moreover we have a canonical decomposition $\mathcal{F}=\bigoplus_{E \in \operatorname{Irr} \mathbf{W}} E_{q} \otimes \rho_{E}$ (as a $\left(\mathcal{H}_{q}, G^{F}\right)$-module), where $\rho_{E}$ is an irreducible representation of $G^{F}$. Now let $\gamma$ be an $F$-stable $G$-conjugacy class in $G$. Then $\mathfrak{B}_{w}^{\gamma}$ has a natural Frobenius map $(g, B) \mapsto(F(g), F(B))$ denoted again by $F$. We compute the number of fixed points of $F: \mathfrak{B}_{w}^{\gamma} \rightarrow \mathfrak{B}_{w}^{\gamma}$ :

$$
\begin{aligned}
& \left|\left(\mathfrak{B}_{w}^{\gamma}\right)^{F}\right|=\sum_{g \in \gamma^{F}} \sharp\left(B \in \mathcal{B}^{F} ;\left(B, g B g^{-1}\right) \in \mathcal{O}_{w}\right) \\
& =\sum_{g \in \gamma^{F}} \operatorname{tr}\left(g T_{w}: \mathcal{F} \rightarrow \mathcal{F}\right)=\sum_{g \in \gamma^{F}} \sum_{E \in \operatorname{Irr} \mathbf{W}} \operatorname{tr}\left(T_{w}, E_{q}\right) \operatorname{tr}\left(g, \rho_{E}\right) .
\end{aligned}
$$

For any $y \in \mathbf{W}$, let $R^{\theta}(y)$ be the virtual representation of $G^{F}$ defined in [DL, 1.9] $\left(\theta\right.$ as in [DL, 1.8]). We have $\rho_{E}=|\mathbf{W}|^{-1} \sum_{y \in \mathbf{W}}\left(\rho_{E}: R^{1}(y)\right) R^{1}(y)+\xi_{E}$, where $\xi_{E}$ is a virtual representation of $G^{F}$ orthogonal to each $R^{\theta}(y)$ and $\left(\rho_{E}: R^{1}(y)\right)$ denotes 
multiplicity. Using the equality

$$
\sum_{g \in \gamma^{F}} \operatorname{tr}\left(g, \xi_{E}\right)=0
$$

(verified below) we deduce that

$$
\left|\left(\mathfrak{B}_{w}^{\gamma}\right)^{F}\right|=\sum_{g \in \gamma^{F}} \sum_{E \in \operatorname{Irr} \mathbf{W}} \operatorname{tr}\left(T_{w}, E_{q}\right)|\mathbf{W}|^{-1} \sum_{y \in \mathbf{W}}\left(\rho_{E}: R^{1}(y)\right) \operatorname{tr}\left(g, R^{1}(y)\right) .
$$

For any $E^{\prime} \in \operatorname{Irr} \mathbf{W}$ we set $R_{E^{\prime}}=|\mathbf{W}|^{-1} \sum_{y^{\prime} \in \mathbf{W}} \operatorname{tr}\left(y^{\prime}, E^{\prime}\right) R^{1}\left(y^{\prime}\right)$ so that $R^{1}(y)=$ $\sum_{E^{\prime} \in \operatorname{Irr} \mathbf{W}} \operatorname{tr}\left(y, E^{\prime}\right) R_{E^{\prime}}$. We have

$$
=\sum_{g \in \gamma^{F}} \sum_{E, E^{\prime}, E^{\prime \prime} \in \operatorname{Irr} \mathbf{W}} \operatorname{tr}\left(T_{w}, E_{q}\right)|\mathbf{W}|^{-1} \sum_{y \in \mathbf{W}} \operatorname{tr}\left(y, E^{\prime}\right) \operatorname{tr}\left(y, E^{\prime \prime}\right)\left(\rho_{E}: R_{E^{\prime}}\right) \operatorname{tr}\left(g, R_{E^{\prime \prime}}\right) .
$$

Hence

(c)

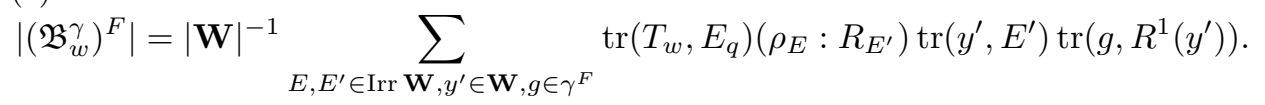

We now verify (b). Let $A$ be the vector space of $G^{F}$-invariant functions $G^{F} \rightarrow \overline{\mathbf{Q}}_{l}$. Let $A_{0}$ be the subspace of $A$ spanned by the functions $f^{\theta}(y)$ (the character of $R^{\theta}(y)$ ) for various $y, \theta$. Let $\chi \in A$ be the characteristic function of the subset $\gamma^{F}$ of $G^{F}$. We must show that if $f \in A$ is orthogonal to $A_{0}$, then it is orthogonal to $\chi$. It is enough to show that $\chi \in A_{0}$. If $G$ is a classical group or if $p$ is not a bad prime for $G$, this follows from results in [L3, L4]; in the general case it is proved by M. Geck $\mathrm{Ge}$, using results in $[\mathrm{L} 3, \mathrm{~L} 4, \mathrm{Sh}]$.

Now assume that $G$ is adjoint of exceptional type, that $p$ is not a bad prime for $G$, that $\gamma \in \underline{G}$ and that $w \in C_{\min }$ where $C \in \underline{\mathbf{W}}_{e l}$. We also assume that $q-1$ is sufficiently divisible. Then (c) becomes

$$
\left|\left(\mathfrak{B}_{w}^{\gamma}\right)^{F}\right|=|\mathbf{W}|^{-1} \sum A_{E, C} \phi_{E, E^{\prime}} a_{E^{\prime}, C^{\prime}} d_{C^{\prime}, C^{\prime \prime}} Q\left(C^{\prime \prime}, \gamma_{0}\right) D\left(\gamma_{0}, \gamma_{0}^{\prime}\right) P\left(\gamma_{0}^{\prime}, \gamma\right),
$$

where the sum is taken over all $E, E^{\prime}$ in $\operatorname{Irr} \mathbf{W}, C^{\prime}, C^{\prime \prime}$ in $\underline{\mathbf{W}}, \gamma_{0}, \gamma_{0}^{\prime}$ in $\underline{\underline{G}}_{0}$ and the notation is as follows.

$\underline{\underline{G}}_{0}$ is the set of $G^{F}$-conjugacy classes of unipotent elements in $G^{F}$. For $\gamma_{0} \in$ $\underline{\underline{G}}_{0}, \gamma^{\prime} \in \underline{\underline{G}}$ we set $D_{\gamma_{0}, \gamma^{\prime}}=\left|\gamma_{0}\right|$ if $\gamma_{0} \subset \gamma^{\prime}$ and $D_{\gamma_{0}, \gamma^{\prime}}=0$ if $\gamma_{0} \not \subset \gamma^{\prime}$. For $C^{\prime}, C^{\prime \prime} \in \underline{\mathbf{W}}$ we set $d_{C^{\prime}, C^{\prime \prime}}=\left|C^{\prime}\right|$ if $C^{\prime}=C^{\prime \prime}$ and $d_{C^{\prime}, C^{\prime \prime}}=0$ if $C^{\prime} \neq C^{\prime \prime}$. For $C^{\prime} \in \underline{\mathbf{W}}, E \in \operatorname{Irr} \mathbf{W}$ we set $A_{E, C^{\prime}}=\operatorname{tr}\left(T_{z}, E_{q}\right), a_{E, C^{\prime}}=\operatorname{tr}(z, E)$, where $z \in C_{\min }^{\prime}$. (Note that $A_{E, C^{\prime}}$ is well defined by GP, 8.2.6(b)].) For $E, E^{\prime} \in \operatorname{Irr} \mathbf{W}$, let $\phi_{E, E^{\prime}}=\left(\rho_{E}: R_{E^{\prime}}\right)$. For $\gamma_{0} \in \underline{G}_{0}$ and $C \in \underline{\mathbf{W}}$, let $Q_{C, \gamma_{0}}=\operatorname{tr}\left(g, R^{1}(y)\right)$, where $g \in \gamma_{0}, y \in C$.

Thus $\left|\left(\mathfrak{B}_{w}^{\gamma}\right)^{F}\right|$ is $|\mathbf{W}|^{-1}$ times the $(C, \gamma)$ entry of the matrix which is the product of matrices

$$
{ }^{t}\left(A_{E, C}\right)\left(\phi_{E, E^{\prime}}\right)\left(a_{E^{\prime}, C^{\prime}}\right)\left(d_{C^{\prime}, C^{\prime \prime}}\right)\left(Q_{C^{\prime \prime}, \gamma_{0}}\right)\left(D_{\gamma_{0}, \gamma}\right) .
$$

Each of these matrices is explicitly known. The matrix $\left(A_{E, C^{\prime}}\right)$ is known from the works of Geck and Geck-Michel (see [GP, 11.5.11]) and is available through the CHEVIE package; the matrix $\left(d_{C^{\prime}, C^{\prime \prime}}\right)$ is available from the same source. The matrix $\left(a_{E, C^{\prime}}\right)$ is the specialization $q=1$ of $\left(A_{E, C^{\prime}}\right)$. The matrix $\phi_{E, E^{\prime}}$ has as entries the coefficients of the "nonabelian Fourier transform" in [L2, 4.15]. The matrix $\left(Q_{C^{\prime \prime}, \gamma_{0}}\right)$ is the matrix of Green functions, known from the work of Shoji 
and Beynon-Spaltenstein. I thank Frank Lübeck for providing tables of Green functions in GAP-format and instructions on how to use them; these tables can now be found at $[\mathrm{Lu}]$; the matrix $\left(D_{\gamma_{0}, \gamma^{\prime}}\right)$ can be extracted from the same source. Thus $\left|\left(\mathfrak{B}_{w}^{\gamma}\right)^{F}\right|$ can be obtained by calculating the product of six (large) explicitly known matrices. The calculation was done using the CHEVIE package; see [Ch]. It turns out that $\left|\left(\mathfrak{B}_{w}^{\gamma}\right)^{F}\right|$ is a polynomial in $q$ with integer coefficients. Note that $\mathfrak{B}_{w}^{\gamma} \neq \emptyset$ if and only if $\left|\left(\mathfrak{B}_{w}^{\gamma}\right)^{F}\right| \neq 0$ for sufficiently large $q$. Thus the condition that $C \dashv \gamma$ can be tested. This can be used to check that Proposition 0.6 holds in our case. (This method is a simplification of the method in [L6, 1.5].)

From the explicit calculations above we see that the following hold when $C$ is elliptic:

(d) If $\gamma=\gamma_{C}, w \in C_{\min }$, then $\left|\left(\mathfrak{B}_{w}^{\gamma}\right)^{F}\right| /\left|G^{F}\right|$ is a polynomial in $q$ with constant term 1. If $C \dashv \gamma, w \in C_{\min }$ but $\gamma \neq \gamma_{C}$, then $\left|\left(\mathfrak{B}_{w}^{\gamma}\right)^{F}\right| /\left|G^{F}\right|$ is a polynomial in $q$ with constant term 0 . If $w \in C_{\min }$, the sum $\sum_{\gamma \in \underline{G}}\left|\left(\mathfrak{B}_{w}^{\gamma}\right)^{F}\right| /\left|G^{F}\right|$ is a palindromic polynomial in $q$ of the form $1+\cdots+q^{\underline{l}(w)-r}$ ( $r$ is the rank of $\left.G_{a d}\right)$; the constant term 1 comes from $\gamma=\gamma_{C}$, and the highest term $q^{\underline{l}(w)-r}$ comes from the regular unipotent class.

We now see that Proposition 0.6 holds. The correspondence $C \mapsto \gamma_{C}$ for $C \in \underline{\mathbf{W}}_{e l}$ is described explicitly in $\S 4.3$.

We expect that (d) also holds for classical types.

1.3. Let $V$ be a $\mathbf{k}$-vector space of finite dimension $\mathbf{n} \geq 3$. We set $\kappa=\kappa_{\mathbf{n}}$. Let $n=(\mathbf{n}-\kappa) / 2$. Assume that $V$ has a fixed bilinear form $():, V \times V \rightarrow \mathbf{k}$ and a fixed quadratic form $Q: V \rightarrow \mathbf{k}$ such that (i) or (ii) below holds:

(i) $Q=0,(x, x)=0$ for all $x \in V, V^{\perp}=0$;

(ii) $Q \neq 0,(x, y)=Q(x+y)-Q(x)-Q(y)$ for $x, y \in V, Q: V^{\perp} \rightarrow \mathbf{k}$ is injective. Here, for any subspace $V^{\prime}$ of $V$ we set $V^{\prime \perp}=\left\{x \in V:\left(x, V^{\prime}\right)=0\right\}$. In case (ii) it follows that $V^{\perp}=0$ unless $\kappa=1$ and $p=2$ in which case $\operatorname{dim} V^{\perp}=1$. An element $g \in G L(V)$ is said to be an isometry if $(g x, g y)=(x, y)$ for all $x, y \in V$ and $Q(g x)=Q(x)$ for all $x \in V$. Let $I s(V)$ be the group of all isometries of $V$ (a closed subgroup of $G L(V))$. A subspace $V^{\prime}$ of $V$ is said to be isotropic if $($,$) and Q$ are zero on $V^{\prime}$. Let $\mathcal{F}$ be the set of all sequences $V_{*}=\left(0=V_{0} \subset V_{1} \subset V_{2} \subset \ldots \subset V_{\mathbf{n}}=V\right)$ of subspaces of $V$ such that $\operatorname{dim} V_{i}=i$ for $i \in[0, \mathbf{n}],\left.Q\right|_{V_{i}}=0$ and $V_{i}^{\perp}=V_{\mathbf{n}-i}$ for all $i \in[0, n]$. (For such a $V_{*}, V_{i}$ is an isotropic subspace for $i \in[0, n]$.) Now $I s(V)$ acts naturally (transitively) on $\mathcal{F}$.

In the remainder of this section we assume that $G$ is the identity component of $I s(V)$.

1.4. Let $W$ be the group of permutations of $[1, \mathbf{n}]$ which commute with the involution $i \mapsto \mathbf{n}-i+1$ of $[1, \mathbf{n}]$. (In particular, if $\kappa=1$, then any permutation in $W$ fixes $n+1$.) Let $V_{*}, V_{*}^{\prime}$ be two sequences in $\mathcal{F}$. As in [L5, 0.4] we define a permutation $a_{V_{*}, V_{*}^{\prime}}: i \mapsto a_{i}$ of $[1, \mathbf{n}]$ as follows. For $i \in[0, \mathbf{n}], j \in[1, \mathbf{n}]$ we set $d_{i j}=$ $\operatorname{dim}\left(V_{i}^{\prime} \cap V_{j}\right) /\left(V_{i}^{\prime} \cap V_{j-1}\right) \in\{0,1\}$. For $i \in[0, \mathbf{n}]$ we set $X_{i}=\left\{j \in[1, \mathbf{n}]: d_{i j}=1\right\}$. We have $\emptyset=X_{0} \subset X_{1} \subset X_{2} \subset \ldots \subset X_{\mathbf{n}}=[1, \mathbf{n}]$ and for $i \in[1, \mathbf{n}]$ there is a unique $a_{i} \in[1, \mathbf{n}]$ such that $X_{i}=X_{i-1} \sqcup\left\{a_{i}\right\}$. Then $i \mapsto a_{i}$ is the required permutation of $[1, \mathbf{n}]$. It belongs to $W$. Moreover,

(a) $\left(V_{*}, V_{*}^{\prime}\right) \mapsto a_{V_{*}, V_{*}^{\prime}}$ defines a bijection from the set of Is $(V)$-orbits on $\mathcal{F} \times \mathcal{F}$ (for the diagonal action) to $W$. 
When $\kappa=0, Q \neq 0$ we define $W^{\prime}$ as the group of even permutations in $W$ (a subgroup of index 2 of $W$ ). For $i \in[1, n-1]$ define $s_{i} \in W$ as a product of two transpositions $i \leftrightarrow i+1, \mathbf{n}+1-i \leftrightarrow \mathbf{n}-i$ (all other entries go to themselves); define $s_{n} \in W$ to be the transposition $n \leftrightarrow \mathbf{n}-n+1$ (all other entries go to themselves). Then $\left(W,\left\{s_{i} ; i \in[1, n]\right\}\right)$ is a Weyl group of type $B_{n}$. If $\kappa=0, Q \neq 0$, we have $s_{i} \in W^{\prime}$ for $i \in[1, n-1]$ and we set $\tilde{s}_{i}=s_{n} s_{i} s_{n} \in W^{\prime}$ for $i \in[1, n-1]$; we have $\tilde{s}_{i}=s_{i}$ if $i<n-1$ and $\left(W^{\prime},\left\{s_{1}, s_{2}, \ldots, s_{n-1}, \tilde{s}_{n-1}\right\}\right)$ is a Weyl group of type $D_{n}$.

1.5. For any $V_{*} \in \mathcal{F}$ we set $B_{V_{*}}=\left\{g \in G: g V_{*}=V_{*}\right\}$, a Borel subgroup of $G$. If $(1-\kappa) Q=0$, then $V_{*} \mapsto B_{V_{*}}$ is an isomorphism $\mathcal{F} \stackrel{\sim}{\longrightarrow} \mathcal{B}$; for $w \in W$ and $V_{*}, V_{*}^{\prime}$ in $\mathcal{F}$ such that $a_{V_{*}, V_{*}^{\prime}}=w$ we have $\left(B_{V_{*}}, B_{V_{*}^{\prime}}\right) \in \mathcal{O}_{f(w)}$ for a well-defined element $f(w) \in \mathbf{W}$ and $w \mapsto f(w)$ is an isomorphism $W \stackrel{\sim}{\longrightarrow} \mathbf{W}$ (as Coxeter groups) by which these two groups are identified. If $(1-\kappa) Q \neq 0, G$ has two orbits on $\mathcal{F}$. Let $\mathcal{F}^{\prime}$ be one of these orbits. Then $V_{*} \mapsto B_{V_{*}}$ is an isomorphism $\mathcal{F}^{\prime} \stackrel{\sim}{\longrightarrow} \mathcal{B}$; for $w \in W^{\prime}$ and $V_{*}, V_{*}^{\prime}$ in $\mathcal{F}$ such that $a_{V_{*}, V_{*}^{\prime}}=w$ and $V_{*} \in \mathcal{F}^{\prime}$ we have $V_{*}^{\prime} \in \mathcal{F}^{\prime}$ and $\left(B_{V_{*}}, B_{V_{*}^{\prime}}\right) \in \mathcal{O}_{f(w)}$ for a well-defined element $f(w) \in \mathbf{W}$. Moreover $w \mapsto f(w)$ is an isomorphism $W^{\prime} \stackrel{\sim}{\longrightarrow} \mathbf{W}$ (as Coxeter groups) by which these two groups are identified.

1.6. Let $\mathcal{P}_{n}$ be the set of sequences $p_{1} \geq p_{2} \geq \cdots \geq p_{\sigma}$ of integers $\geq 1$ such that $p_{1}+p_{2}+\cdots+p_{\sigma}=n$. Let $\mathcal{P}_{n}^{+}$be the set of sequences $p_{1} \geq p_{2} \geq \cdots \geq p_{\sigma}$ in $\mathcal{P}_{n}$ such that $\kappa_{\sigma}=0$. For any $r \in[1, \sigma]$ we set $p_{\leq r}=\sum_{r^{\prime} \in[1, r]} p_{r^{\prime}}, p_{<r}=\sum_{r^{\prime} \in[1, r-1]} p_{r^{\prime}}$, $p_{>r}=\sum_{r^{\prime} \in[r+1, \sigma]} p_{r^{\prime}}$. For $p_{*} \in \mathcal{P}_{n}$ let $w_{p_{*}} \in W$ be the permutation of $[1, \mathbf{n}]$ given by

$$
\begin{aligned}
& 1 \mapsto 2 \mapsto \ldots \mapsto p_{1} \mapsto \mathbf{n} \mapsto \mathbf{n}-1 \mapsto \ldots \mapsto \mathbf{n}-p_{1} \mapsto 1, \\
& p_{1}+1 \mapsto p_{1}+2 \mapsto \ldots \mapsto p_{1}+p_{2} \mapsto \mathbf{n}-p_{1}-1 \mapsto \mathbf{n}-p_{1}-2 \mapsto \ldots \\
& \mapsto \mathbf{n}-p_{1}-p_{2} \mapsto p_{1}+1, \\
& \ldots \\
& p_{<\sigma}+1 \mapsto p_{<\sigma}+2 \mapsto \ldots \mapsto p_{<\sigma}+p_{\sigma} \mapsto \mathbf{n}-p_{<\sigma}-1 \\
& \mapsto \mathbf{n}-p_{<\sigma}-2 \mapsto \ldots \mapsto \mathbf{n}-p_{<\sigma}-p_{\sigma} \mapsto p_{<\sigma}+1, \\
& \text { and, if } \kappa=1, \\
& p_{n+1} \mapsto p_{n+1} .
\end{aligned}
$$

Let $C_{p_{*}}$ be the conjugacy class of $w_{p_{*}}$ in $W$. If $(1-\kappa) Q=0$, then $w_{p_{*}} \in \underline{\mathbf{W}}_{e l}$ and $p_{*} \mapsto C_{p_{*}}$ is a bijection $\mathcal{P}_{n} \stackrel{\sim}{\longrightarrow} \underline{\mathbf{W}}_{e l}$. If $(1-\kappa) Q \neq 0$ and $p_{*} \in \mathcal{P}_{n}^{+}$, then $w_{p_{*}} \in \underline{\mathbf{W}}_{e l}$; we denote by $C_{p_{*}}^{\prime}$ the conjugacy class of $w_{p_{*}}$ in $W^{\prime}$. Then $p_{*} \mapsto C_{p_{*}}^{\prime}$ is a bijection $\mathcal{P}_{n}^{+} \stackrel{\sim}{\longrightarrow} \underline{\mathbf{W}}_{e l}$.

For any $p_{1} \geq p_{2} \geq \cdots \geq p_{\sigma}$ in $\mathcal{P}_{n}$ we define a function $\psi:[1, \sigma] \rightarrow\{-1,0,1\}$ as follows.

(i) If $t \in[1, \sigma]$ is odd and $p_{t}<p_{x}$ for any $x \in[1, t-1]$, then $\psi(t)=1$;

(ii) if $t \in[1, \sigma]$ is even and $p_{x}<p_{t}$ for any $x \in[t+1, \sigma]$, then $\psi(t)=-1$;

(iii) for all other $t \in[1, \sigma]$ we have $\psi(t)=0$.

For any integer $a$ such that $1 \leq 2 a<2 a+1 \leq \sigma$ we have $\psi(2 a)+\psi(2 a+1)=0$. (Indeed if $p_{2 a}>p_{2 a+1}$, then $\psi(2 a)+\psi(2 a+1)=-1+1=0$; if $p_{2 a}=p_{2 a+1}$, then $\psi(2 a)+\psi(2 a+1)=0+0=0$.) Also $\psi(1)=1$. Hence

if $h \in[1, \sigma]$ is odd, then $\sum_{r \in[1, h]} \psi(r)=1$;

if $h \in[1, \sigma]$ is even, then $\sum_{r \in[1, h]} \psi(r)=1+\psi(h)$. 
We have $\psi(\sigma)=-1$ if $\sigma$ is even. Hence

$$
\sum_{t \in[1, \sigma]} \psi(t)=\kappa_{\sigma}
$$

\section{EXCELLENT DECOMPOSITIONS AND UNIPOTENT ELEMENTS}

2.1. Let $C \in \underline{\mathbf{W}}_{e l}$ and let $w \in C$. Let $\rho=|S|$. An excellent decomposition of $w$ is a sequence

$$
\begin{aligned}
& s_{1}^{1}, s_{2}^{1}, \ldots, s_{q_{1}}^{1}, s_{q_{1}+1}^{1}, s_{q_{1}}^{1}, \ldots, s_{2}^{1}, s_{1}^{1}, s_{1}^{2}, s_{2}^{2}, \ldots, s_{q_{2}}^{2}, s_{q_{2}+1}^{2}, s_{q_{2}}^{2}, \ldots, s_{2}^{2}, s_{1}^{2}, \ldots \\
& s_{1}^{\rho}, s_{2}^{\rho}, \ldots, s_{q_{\rho}}^{\rho}, s_{q_{\rho}+1}^{\rho}, s_{q_{\rho}}^{\rho}, \ldots, s_{2}^{\rho}, s_{1}^{\rho}
\end{aligned}
$$

in $S$ (the upper scripts are not powers) consisting of $\sum_{k \in[1, \rho]}\left(2 q_{k}+1\right)=\underline{l}(w)$ terms, such that

$$
w=\left(s_{1}^{1} s_{2}^{1} \ldots s_{q_{1}}^{1} s_{q_{1}+1}^{1} s_{q_{1}}^{1} \ldots s_{2}^{1} s_{1}^{1}\right)\left(s_{1}^{2} s_{2}^{2} \ldots s_{q_{2}}^{2} s_{q_{2}+1}^{2} s_{q_{2}}^{2} \ldots s_{2}^{2} s_{1}^{2}\right) \ldots
$$

We sometimes refer to (a) as an excellent decomposition of $w$. It is a reduced expression of a special kind for $w$. It appears that

(b) for any $C \in \underline{\mathbf{W}}_{e l}$, at least one element $w \in C_{\min }$ admits an excellent decomposition.

For example if $C=C_{c o x}$, then for any $w \in C_{\min }$ any reduced expression of $w$ is an excellent decomposition. In particular (b) holds when $G$ is almost simple of type $A_{n}$. When $G$ is simple of type $G_{2}$, the excellent decompositions (1)(2),(121)(2),(12121)(2) account for the 3 elliptic conjugacy classes in W with $S=\{1,2\}$. In type $F_{4}$, the excellent decompositions

$$
\begin{aligned}
& \text { (1)(2)(3)(4); } \quad(1)(232)(3)(4) ; \quad(121)(323)(4)(3) ; \quad(1)(2)(3234323)(4) ; \\
& \text { (4)(3)(2321232)(1); (12321)(23432)(3)(4); } \quad(2)(12321)(3234323)(4) \text {; } \\
& (2324312134232)(3)(1)(4) ; \quad(432134232431234)(12321)(232)(3)
\end{aligned}
$$

(notation of [GP p.407]) account for the 9 elliptic conjugacy classes in $\mathbf{W}$; in type $E_{6}$, the excellent decompositions

$$
\begin{aligned}
& \begin{array}{l}
(1)(2)(3)(4)(5)(6) ; \quad(1)(3)(4)(2)(454)(6) ; \quad(1)(3)(4)(2345432)(6)(5) ; \\
(1)(2)(3)(432454234)(5)(6) ; \quad(4354132456542314534)(2)(1)(3)(5)(6)
\end{array}
\end{aligned}
$$

(notation of [GP p.407]) account for the 5 elliptic conjugacy classes in W. Note that some elements in $C_{\mathrm{min}}$ might not admit an excellent decomposition (example: the element 324312 in type $F_{4}$ ). In SS2.2, 2.3 we will verify (b) for $G$ of type $B_{n}, C_{n}, D_{n}$. 
Note added November 16, 2010. After this paper was submitted, M. Geck ("Some applications of CHEVIE to the theory of algebraic groups", arXiv:1008.1464) has verified (b) with the aid of a computer in the remaining cases $E_{7}, E_{8}$.

2.2. Assume that the notation is as in $\S 1.3$ and that $(1-\kappa) Q=0$. Let $p_{*}=\left(p_{1} \geq\right.$ $\left.p_{2} \geq \cdots \geq p_{\sigma}\right) \in \mathcal{P}_{n}$. The following is an excellent decomposition of $w=w_{p_{*}}^{-1}$ (see $1.6(\mathrm{a}))$ in $\mathbf{W}$ :

(a) $\quad w=\left(s_{n}\right)\left(s_{n-1}\right) \ldots\left(s_{n-p_{\sigma}+1}\right) \times$

$$
\begin{aligned}
& \left(s_{n-p_{\sigma}} \ldots s_{n-1} s_{n} s_{n-1} \ldots s_{n-p_{\sigma}}\right)\left(s_{n-p_{\sigma}-1}\right)\left(s_{n-p_{\sigma}-2}\right) \ldots\left(s_{n-p_{\sigma}-p_{\sigma-1}+1}\right) \times \\
& \left(s_{n-p_{\sigma}-p_{\sigma-1}} \ldots s_{n-1} s_{n} s_{n-1} \ldots s_{n-p_{\sigma}-p_{\sigma-1}}\right)\left(s_{n-p_{\sigma}-p_{\sigma-1}-1}\right)\left(s_{n-p_{\sigma}-p_{\sigma-1}-2}\right) \\
& \ldots\left(s_{n-p_{\sigma}-p_{\sigma-1}-p_{\sigma-2}+1}\right) \times \\
& \ldots \\
& \left(s_{n-p_{\sigma}-\cdots-p_{2}} \ldots s_{n-1} s_{n} s_{n-1} \ldots s_{n-p_{\sigma}-\cdots-p_{2}}\right)\left(s_{n-p_{\sigma}-\cdots-p_{2}-1}\right) \times \\
& \left(s_{n-p_{\sigma}-\cdots-p_{2}-2}\right) \ldots\left(s_{n-p_{\sigma}-\cdots-p_{1}+1}\right) .
\end{aligned}
$$

Note that $\underline{l}(w)=2 \sum_{v=1}^{\sigma-1} v p_{v+1}+n$. Thus $w$ has minimal length in its conjugacy class in W (see [GP, 3.4]). We see that 2.1(b) holds for $G$ of type $B_{n}$ or $C_{n}$.

In the case where $p_{*} \in \mathcal{P}_{n}^{+}$we define another excellent decomposition of $w=w_{p_{*}}^{-1}$ (only the parantheses differ from the previous excellent decomposition):

(b) $\quad w=\left(s_{n} s_{n-1} \ldots s_{n-p_{\sigma}} \ldots s_{n-1} s_{n}\right)\left(s_{n-1}\right)\left(s_{n-2}\right) \ldots\left(s_{n-p_{\sigma}-p_{\sigma-1}+1}\right) \times$

$$
\begin{aligned}
& \left(s_{n-p_{\sigma}-p_{\sigma-1}} \ldots s_{n-1} s_{n} s_{n-1} \ldots s_{n-p_{\sigma}-p_{\sigma-1}-p_{\sigma-2}} \ldots s_{n-1} s_{n} s_{n-1} \ldots\right. \\
& \left.s_{n-p_{\sigma}-p_{\sigma-1}}\right)\left(s_{n-p_{\sigma}-p_{\sigma-1}-1}\right)\left(s_{n-p_{\sigma}-p_{\sigma-1}-2}\right) \ldots\left(s_{n-p_{\sigma}-p_{\sigma-1}-p_{\sigma-2}-p_{\sigma-3}+1}\right) \times \\
& \ldots \\
& \left(s_{n-p_{\sigma}-\cdots-p_{3}} \ldots s_{n-1} s_{n} s_{n-1} \ldots s_{n-p_{\sigma}-\cdots-p_{2}} \ldots s_{n-1} s_{n} s_{n-1} \ldots\right. \\
& \left.s_{n-p_{\sigma}-\cdots-p_{3}}\right)\left(s_{n-p_{\sigma}-\cdots-p_{3}-1}\right)\left(s_{n-p_{\sigma}-\cdots-p_{3}-2}\right) \ldots\left(s_{n-p_{\sigma}-\cdots-p_{1}+1}\right) .
\end{aligned}
$$

2.3. Assume that the notation is as in $\S 1.3$ and that $(1-\kappa) Q \neq 0$. Let $p_{*}=\left(p_{1} \geq\right.$ $\left.p_{2} \geq \cdots \geq p_{\sigma}\right) \in \mathcal{P}_{n}^{+}$. The excellent decomposition $2.2(\mathrm{~b})$ in $W$ gives rise to an excellent decomposition of $w=w_{p_{*}}^{-1}$ in $W^{\prime}=\mathbf{W}$ :

$$
\begin{aligned}
& w=\left(\tilde{s}_{n-1} \ldots \tilde{s}_{n-p_{\sigma}} \ldots \tilde{s}_{n-1}\right)\left(s_{n-1}\right)\left(s_{n-2}\right) \ldots\left(s_{n-p_{\sigma}-p_{\sigma-1}+1}\right) \times \\
& \left(s_{n-p_{\sigma}-p_{\sigma-1}} \ldots s_{n-1} \tilde{s}_{n-1} \ldots \tilde{s}_{n-p_{\sigma}-p_{\sigma-1}-p_{\sigma-2}} \ldots \tilde{s}_{n-1} s_{n-1} \ldots\right. \\
& \left.s_{n-p_{\sigma}-p_{\sigma-1}}\right)\left(s_{n-p_{\sigma}-p_{\sigma-1}-1}\right)\left(s_{n-p_{\sigma}-p_{\sigma-1}-2}\right) \ldots\left(s_{n-p_{\sigma}-p_{\sigma-1}-p_{\sigma-2}-p_{\sigma-3}+1}\right) \times \\
& \ldots \\
& \left(s_{n-p_{\sigma}-\cdots-p_{3}} \ldots s_{n-1} \tilde{s}_{n-1} \ldots \tilde{s}_{n-p_{\sigma}-\cdots-p_{2}} \ldots \tilde{s}_{n-1} s_{n-1} \ldots\right. \\
& \left.s_{n-p_{\sigma}-\cdots-p_{3}}\right)\left(s_{n-p_{\sigma}-\cdots-p_{3}-1}\right)\left(s_{n-p_{\sigma}-\cdots-p_{3}-2}\right) \ldots\left(s_{n-p_{\sigma}-\cdots-p_{1}+1}\right) .
\end{aligned}
$$

Now the length of $w$ in $W^{\prime}$ is equal to the length of $w$ in $W$ minus $\sigma$; hence it is $2 \sum_{v=1}^{\sigma-1} v p_{v+1}+n-\sigma$. Thus $w$ has minimal length in its conjugacy class in $\mathbf{W}=W^{\prime}$ (see [GP, 3.4]). We see that 2.1(b) holds for $G$ of type $D_{n}$.

2.4. We return to the general case. We choose a Borel subgroup $B$ of $G$ and a Borel subgroup $B^{\prime}$ opposed to $B$. Let $T=B \cap B^{\prime}$, a maximal torus $T$ of $G$. Let $N(T)$ be the normalizer of $T$ in $G$. Let $U^{\prime}=U_{B^{\prime}}$. For any $s \in S$, let $P_{s}$ be the parabolic subgroup of type $\{s\}$ that contains $B$. Note that $U_{s}^{\prime}:=U^{\prime} \cap P_{s}$ is isomorphic to k. 
Let $t \mapsto y_{s}(t)$ be an isomorphism of algebraic groups $\mathbf{k} \stackrel{\sim}{\longrightarrow} U_{s}^{\prime}$. Let $\dot{s} \in N T$ be an element such that $\left(B, \dot{s} B \dot{s}^{-1}\right) \in \mathcal{O}_{s}$.

Let $C \in \underline{\mathbf{W}}_{e l}$ and let 2.1(a) be an excellent decomposition of an element $w \in$ $C_{\min }$. Let $c_{1}, c_{2}, \ldots, c_{\rho}$ be elements of $\mathbf{k}^{*}$. We set

$$
\begin{aligned}
& u_{w}=\left(\dot{s}_{1}^{1} \dot{s}_{2}^{1} \ldots \dot{s}_{q_{1}}^{1} y_{s_{q_{1}+1}^{1}}\left(c_{1}\right)\left(\dot{s}_{q_{1}}^{1}\right)^{-1} \ldots\left(\dot{s}_{2}^{1}\right)^{-1}\left(\dot{s}_{1}^{1}\right)^{-1}\right) \times \\
& \left(\dot{s}_{1}^{2} \dot{s}_{2}^{2} \ldots \dot{s}_{q_{2}}^{2} y_{q_{q_{2}+1}^{2}}\left(c_{2}\right)\left(\dot{s}_{q_{2}}^{2}\right)^{-1} \ldots\left(\dot{s}_{2}^{2}\right)^{-1}\left(\dot{s}_{1}^{2}\right)^{-1}\right) \ldots \\
& \left(\dot{s}_{1}^{\rho} \dot{s}_{2}^{\rho} \ldots \dot{s}_{q_{\rho}}^{\rho} y_{s_{q_{\rho}+1}^{\rho}}\left(c_{\rho}\right)\left(\dot{s}_{q_{\rho}}^{\rho}\right)^{-1} \ldots\left(\dot{s}_{2}^{\rho}\right)^{-1}\left(\dot{s}_{1}^{\rho}\right)^{-1}\right) .
\end{aligned}
$$

We have $u_{w} \in U^{\prime}$; thus, $u_{w}$ is unipotent. Since $y_{s_{q_{h}+1}^{h}}\left(c_{h}\right) \in B \dot{s}_{q_{h}+1}^{h} B$ and since 2.1(a) is a reduced expression for $w$, we see (using properties of the Bruhat decomposition) that

$$
\left(B, u_{w} B u_{w}^{-1}\right) \in \mathcal{O}_{w} .
$$

Much of the remainder of this section is devoted to computing $u_{w}$ in some cases arising from classical groups.

2.5. Assume that the notation is as in $\S 1.3$. In the remainder of this section we assume that $(\kappa-1) Q=0$. We can find (and fix) a basis of $V$ consisting of vectors $e_{i}, e_{i}^{\prime}(i \in[1, n])$ and (if $\kappa=1$ ) of $e_{0}$, such that $\left(e_{i}, e_{j}^{\prime}\right)=\delta_{i, j}$ for $i, j \in[1, n]$, $Q\left(e_{i}\right)=Q\left(e_{i}^{\prime}\right)=0$ for $i \in[1, n]$ and (if $\left.\kappa=1\right),\left(e_{i}, e_{0}\right)=\left(e_{i}^{\prime}, e_{0}\right)$ for $i \in[1, n]$, $\left(e_{0}, e_{0}\right)=2, Q\left(e_{0}\right)=1$.

For $i \in[1, n]$ let $\mathfrak{V}_{i}$ be the span of $e_{1}, e_{2}, \ldots, e_{i}$ and let $\mathfrak{V}_{i}^{\prime}$ be the span of $e_{1}^{\prime}, e_{2}^{\prime}, \ldots, e_{i}^{\prime}$. Let $B$ (resp. $\left.B^{\prime}\right)$ be the set of all $g \in G$ such that for any $i \in[1, n]$, we have $g \mathfrak{V}_{i}=\mathfrak{V}_{i}\left(\right.$ resp. $\left.g \mathfrak{V}_{i}^{\prime}=\mathfrak{V}_{i}^{\prime}\right)$. Note that $B, B^{\prime}$ are opposed Borel subgroups of $G$.

We will sometime specify a linear map $V \rightarrow V$ by indicating its effect on a part of a basis of $V$ with the understanding that the remaining basis elements are sent to themselves. Thus for $h \in[1, n-1]$ we define $y_{s_{h}}(a) \in G L(V)$ by $\left[e_{h} \mapsto e_{h}+a e_{h+1}, e_{h+1}^{\prime} \mapsto e_{h+1}^{\prime}-a e_{h}^{\prime}\right](a \in \mathbf{k})$. In the case where $\kappa=0, Q=0$ we define $y_{s_{n}}(a) \in G L(V)$ by $\left[e_{n} \mapsto e_{n}-a e_{n}^{\prime}\right](a \in \mathbf{k})$. In the case where $\kappa=1, Q \neq 0$ we define $y_{s_{n}}(a) \in G L(V)$ by $\left[e_{n} \mapsto e_{n}+a e_{0}-a^{2} e_{n}^{\prime}, e_{0} \mapsto e_{0}-2 a e_{n}^{\prime}\right](a \in \mathbf{k})$. In both cases we have $y_{s_{h}}(a) \in G$. Note that for $s=s_{h} \in S, y_{s_{h}}: \mathbf{k} \rightarrow U_{s}^{\prime}$ is as in $\S 2.4$.

Let $p_{*}=\left(p_{1} \geq p_{2} \geq \cdots \geq p_{\sigma}\right) \in \mathcal{P}_{n}$ and let $w=w_{p_{*}}^{-1}$ with $w_{p_{*}}$ as in $\S 1.6$. We define $u_{w}$ as in $\S 2.4(\mathrm{a})$ in terms of $c_{1}, c_{2}, \ldots, c_{\rho}$ in $\mathbf{k}^{*}$ and the excellent decomposition $2.2\left(\right.$ a) of $w$. Let $N=u_{w}^{-1}-1 \in \operatorname{End}(V)$.

2.6. Assume now that $\kappa=0, Q=0$. From the definitions, for a suitable choice of $c_{1}, c_{2}, \ldots, c_{\rho}, u_{w}^{-1}$ is the product over $h \in[1, \sigma]$ of the linear maps

$$
\begin{aligned}
& {\left[e_{n-p_{>h}} \mapsto e_{n-p_{>h}}-e_{n-p_{>h}}^{\prime}+e_{n-p_{>h}-1}^{\prime}-e_{n-p_{>h}-2}^{\prime}+\cdots+(-1)^{p_{h}} e_{n-p_{>h}-p_{h}+1}^{\prime},\right.} \\
& e_{n-p_{>h}-1} \mapsto e_{n-p_{>h}-1}+e_{n-p_{>h}}, e_{n-p_{>h}-2} \mapsto e_{n-p_{>h}-2}+e_{n-p_{>h}-1}, \\
& \ldots, e_{n-p_{>h}-p_{h}+1} \mapsto e_{n-p_{>h}-p_{h}+1}+e_{n-p_{>h}-p_{h}+2}, \\
& e_{n-p>h}^{\prime} \mapsto e_{n-p_{>h}}^{\prime}-e_{n-p_{>h}-1}^{\prime}+\cdots+(-1)^{p_{h}-1} e_{n-p_{>h}-p_{h}+1}^{\prime}, \\
& e_{n-p_{>h}-1}^{\prime} \mapsto e_{n-p_{>h}-1}^{\prime}-e_{n-p_{>h}-2}^{\prime}+\cdots+(-1)^{p_{h}-2} e_{n-p_{>h}-p_{h}+1}^{\prime}, \\
& \left.\ldots, e_{n-p_{>h}-p_{h}+2}^{\prime} \mapsto e_{n-p_{>h}-p_{h}+2}^{\prime}-e_{n-p_{>h}-p_{h}+1}^{\prime}\right] .
\end{aligned}
$$


Hence $u_{w}^{-1}$ is given by

$$
\begin{aligned}
& e_{i} \mapsto e_{i}+e_{i+1} \text { if } i \in[1, n], i \notin\left\{p_{1}, p_{1}+p_{2}, \ldots, p_{1}+p_{2}+\cdots+p_{\sigma}\right\}, \\
& e_{p_{1}+p_{2}+\cdots+p_{r}} \mapsto e_{p_{1}+p_{2}+\cdots+p_{r}}+\sum_{v \in\left[1, p_{r}\right]}(-1)^{v} e_{p_{1}+p_{2}+\cdots+p_{r}-v+1}^{\prime} \text { if } r \in[1, \sigma], \\
& e_{p_{1}+p_{2}+\cdots+p_{r}-j}^{\prime} \mapsto \sum_{v \in\left[1, p_{r}-j\right]}(-1)^{v} e_{p_{1}+p_{2}+\cdots+p_{r}-j-v+1}^{\prime} \text { if } r \in[1, \sigma], j \in\left[0, p_{r}-2\right], \\
& e_{i_{1}+i_{2}+\cdots+p_{r-1}+1}^{\prime} \mapsto e_{p_{1}+p_{2}+\cdots+p_{r-1}+1}^{\prime} \text { if } r \in[1, \sigma] .
\end{aligned}
$$

We set

$$
\begin{aligned}
& e_{j}^{t}=e_{p_{1}+\cdots+p_{t-1}+j}\left(t \in[1, \sigma], j \in\left[1, p_{t}\right]\right), \\
& e_{1}^{\prime t}=\sum_{v \in\left[1, p_{t}\right]}(-1)^{v} e_{p_{1}+p_{2}+\cdots+p_{t}-v+1}^{\prime}(t \in[1, \sigma]), \\
& e_{j}^{\prime t}=N^{j-1} e_{1}^{\prime t}(t \in[1, \sigma], j \geq 2) .
\end{aligned}
$$

Note that $e_{j}^{\prime t}=0$ if $j>p_{t}$. Clearly for any $t \in[1, \sigma]$, the elements $e_{j}^{\prime t}\left(j \in\left[1, p_{t}\right]\right)$ span the same subspace as the elements $e_{p_{1}+p_{2}+\cdots+p_{t}-v+1}^{\prime}\left(v \in\left[1, p_{t}\right]\right)$. It follows that $e_{j}^{t}, e_{j}^{\prime t}\left(t \in[1, \sigma], j \in\left[1, p_{t}\right]\right)$ form a basis of $V$. In this basis the action of $N$ is given by

$$
e_{1}^{t} \mapsto e_{2}^{t} \mapsto \ldots \mapsto e_{p_{t}}^{t} \mapsto e_{1}^{\prime t} \mapsto e_{2}^{\prime t} \mapsto \ldots \mapsto e_{p_{t}}^{\prime t} \mapsto 0 \text { if } t \in[1, \sigma]
$$

Thus the Jordan blocks of $N: V \rightarrow V$ have sizes $2 p_{1}, 2 p_{2}, \ldots, 2 p_{\sigma}$.

2.7. Assume now that $\kappa=1, Q \neq 0$. From the definitions, for a suitable choice of $c_{1}, c_{2}, \ldots, c_{\rho}, u_{w}^{-1}$ is the product over $h \in[1, \sigma]$ of the linear maps

$$
\begin{aligned}
& {\left[e_{n-p_{>h}} \mapsto e_{n-p_{>h}}+e_{0}-e_{n-p_{>h}}^{\prime}+e_{n-p_{>h}-1}^{\prime}-e_{n-p_{>h}-2}^{\prime}+\cdots+\right.} \\
& (-1)^{p_{h}} e_{n-p_{>h}-p_{h}+1}^{\prime}, \\
& e_{n-p_{>h}-1} \mapsto e_{n-p_{>h}-1}+e_{n-p_{>h}}, e_{n-p_{>h}-2} \mapsto e_{n-p_{>h}-2}+e_{n-p_{>h}-1}, \\
& \ldots, e_{n-p_{>h}-p_{h}+1} \mapsto e_{n-p_{>h}-p_{h}+1}+e_{n-p_{>h}-p_{h}+2}, \\
& e_{0} \mapsto e_{0}-2 e_{n-p_{>h}}^{\prime}+2 e_{n-p_{>h}-1}^{\prime}-2 e_{n-p_{>h}-2}^{\prime}+\cdots+(-1)^{p_{h}} 2 e_{n-p_{>h}-p_{h}+1}^{\prime}, \\
& e_{n-p_{>h}}^{\prime} \mapsto e_{n-p_{>h}}^{\prime}-e_{n-p_{>h}-1}^{\prime}+\cdots+(-1)^{p_{h}-1} e_{n-p_{>h}-p_{h}+1}^{\prime}, \\
& e_{n-p_{>h}-1}^{\prime} \mapsto e_{n-p_{>h}-1}^{\prime}-e_{n-p_{>h}-2}^{\prime}+\cdots+(-1)^{p_{h}-2} e_{n-p_{>h}-p_{h}+1}^{\prime}, \\
& \left.\ldots, e_{n-p_{>h}-p_{h}+2}^{\prime} \mapsto e_{n-p_{>h}-p_{h}+2}^{\prime}-e_{n-p_{>h}-p_{h}+1}^{\prime}\right] .
\end{aligned}
$$


Hence $u_{w}^{-1}$ is given by

$$
\begin{aligned}
& e_{i} \mapsto e_{i}+e_{i+1} \text { if } i \in[1, n], i \notin\left\{p_{1}, p_{1}+p_{2}, \ldots, p_{1}+p_{2}+\cdots+p_{\sigma}\right\}, \\
& e_{p_{1}+p_{2}+\cdots+p_{r}} \mapsto e_{p_{1}+p_{2}+\cdots+p_{r}}+e_{0}+\sum_{v \in\left[1, p_{r}\right]}(-1)^{v} e_{p_{1}+p_{2}+\cdots+p_{r}-v+1}^{\prime} \\
& +2 \sum_{v \in\left[1, p_{r-1}\right]}(-1)^{v} e_{p_{1}+p_{2}+\cdots+p_{r-1}-v+1}^{\prime}+\cdots+2 \sum_{v \in\left[1, p_{1}\right]}(-1)^{v} e_{p_{1}-v+1}^{\prime} \text { if } r \in[1, \sigma], \\
& e_{0} \mapsto e_{0}+2 \sum_{v \in\left[1, p_{\sigma}\right]}(-1)^{v} e_{p_{1}+p_{2}+\cdots+p_{\sigma}-v+1}^{\prime} \\
& +2 \sum_{v \in\left[1, p_{\sigma-1}\right]}(-1)^{v} e_{p_{1}+p_{2}+\cdots+p_{\sigma-1}-v+1}^{\prime}+\cdots+2 \sum_{v \in\left[1, p_{1}\right]}(-1)^{v} e_{p_{1}-v+1}^{\prime}, \\
& e_{p_{1}+p_{2}+\cdots+p_{r}-j}^{\prime} \mapsto \sum_{v \in\left[1, p_{r}-j\right]}(-1)^{v} e_{p_{1}+p_{2}+\cdots+p_{r}-j-v+1}^{\prime} \text { if } r \in[1, \sigma], j \in\left[0, p_{r}-2\right], \\
& e_{i_{1}+i_{2}+\cdots+p_{r-1}+1}^{\prime} \mapsto e_{p_{1}+p_{2}+\cdots+p_{r-1}+1}^{\prime} \text { if } r \in[1, \sigma] .
\end{aligned}
$$

We set

$$
\begin{aligned}
e_{j}^{t} & =e_{p_{1}+\cdots+p_{t-1}+j}\left(t \in[1, \sigma], j \in\left[1, p_{t}\right]\right), \\
e_{1}^{\prime t} & =\sum_{v \in\left[1, p_{t}\right]}(-1)^{v} e_{p_{1}+p_{2}+\cdots+p_{t}-v+1}^{\prime}(t \in[1, \sigma]), \\
e_{j}^{t} & =N^{j-1} e_{1}^{t}(t \in[1, \sigma], j \geq 2) .
\end{aligned}
$$

Note that $e^{\prime t}{ }_{j}=0$ if $j>p_{t}$. Clearly for any $t \in[1, \sigma]$, the elements $e^{\prime t}{ }_{j}\left(j \in\left[1, p_{t}\right]\right)$ span the same subspace as the elements $e_{p_{1}+p_{2}+\cdots+p_{t}-v+1}^{\prime}\left(v \in\left[1, p_{t}\right]\right)$. It follows that $e_{j}^{t}, e_{j}^{t}\left(t \in[1, \sigma], j \in\left[1, p_{t}\right]\right)$ and $e_{0}$ form a basis of $V$. In this basis the action of $N$ is given by

$$
\begin{aligned}
& e_{j}^{t} \mapsto e_{j+1}^{t}\left(t \in[1, \sigma], j \in\left[1, p_{t}-1\right]\right), \\
& e_{p_{t}}^{t} \mapsto e_{0}+e_{1}^{\prime t}+2 e_{1}^{\prime t-1}+\cdots+2 e_{1}^{\prime 1}(t \in[1, \sigma]), \\
& e_{0} \mapsto 2 e_{1}^{1}+2 e_{1}^{\prime 2}+\cdots+2 e_{1}^{\prime \sigma}, \\
& e_{j}^{\prime t} \mapsto e_{j+1}^{t}\left(t \in[1, \sigma], j \in\left[1, p_{t}-1\right]\right), \\
& e_{p_{t}}^{\prime t} \mapsto 0(t \in[1, \sigma]) .
\end{aligned}
$$

For $t \in[1, \sigma], j \geq 1$, we set $f_{j}^{t}=e_{j}^{t}+2 \sum_{t^{\prime} \in[t+1, s]} e_{j}^{\prime t^{\prime}}(t \in[1, \sigma])$ and $\epsilon=e_{0}+$ $2 \sum_{t \in[1, \sigma]} e_{1}^{t}$. Clearly,

(a) $e_{j}^{t}, f_{j}^{t}\left(t \in[1, \sigma], j \in\left[1, p_{t}\right]\right)$ and $\epsilon$ form a basis of $V$. In this basis the action of $N$ is given by

$$
\begin{aligned}
& e_{j}^{t} \mapsto e_{j+1}^{t}\left(t \in[1, \sigma], j \in\left[1, p_{t}-1\right]\right), \\
& e_{p_{t}}^{t} \mapsto \epsilon-f_{1}^{t}(t \in[1, \sigma]), \\
& \epsilon \mapsto 2 z_{1}+2 z_{2}, \\
& f_{j}^{t} \mapsto f_{j+1}^{t}\left(t \in[1, \sigma], j \in\left[1, p_{t}-1\right]\right), \\
& f_{p_{t}}^{t} \mapsto 0,
\end{aligned}
$$


where for $j \geq 1$ we set

$$
z_{j}=-\sum_{t \in[1, \sigma]}(-1)^{t} f_{j}^{t}
$$

(We use that $-\sum_{t \in[1, \sigma]}(-1)^{t} f_{1}^{t}=\sum_{t \in[1, \sigma]} e_{1}^{\prime t}$.) In the case where $\kappa_{\sigma}=0$ we set

$$
\Xi=-2 \sum_{x \in[1, \sigma]}(-1)^{x} e_{p_{x}}^{x}+\epsilon-2 z_{1}
$$

We have

$$
\begin{aligned}
\Xi & =-2 \sum_{x \in[1, \sigma]}(-1)^{x} e_{p_{x}}^{x}+e_{0}+2 \sum_{t \in[1, \sigma]} e_{1}^{\prime t}+2 \sum_{t \in[1, \sigma]}(-1)^{t} f_{1}^{t} \\
& =-2 \sum_{x \in[1, \sigma]}(-1)^{x} e_{p_{x}}^{x}+e_{0} .
\end{aligned}
$$

From the last expression for $\Xi$ we see that $Q(\Xi)=1$.

2.8. In the setup of $\S 2.7$ we assume that $p=2$. Then the action of $N$ in the basis 2.7 (a) is given by

$$
e_{1}^{t} \mapsto e_{2}^{t} \mapsto \ldots \mapsto e_{p_{t}}^{t} \mapsto f_{1}^{t} \mapsto f_{2}^{t} \mapsto \ldots \mapsto f_{p_{t}}^{t} \mapsto 0 \text { if } t \in[1, \sigma] ; \epsilon \mapsto 0 .
$$

Thus the Jordan blocks of $N: V \rightarrow V$ have sizes $2 p_{1}, 2 p_{2}, \ldots, 2 p_{\sigma}, 1$.

2.9. In the setup of $\S 2.7$ we assume that $p \neq 2$. For $t \in[1, \sigma], j \geq 1$, we set $E_{j}^{t}=$ $N^{j-1} e_{1}^{t}$. Let $\mathcal{V}$ be the subspace of $V$ spanned by the vectors $E_{j}^{t}(t \in[1, \sigma], j \geq 1)$. Clearly, $N \mathcal{V} \subset \mathcal{V}$. We show:

(i) if $\kappa_{\sigma}=1$, then $\mathcal{V}=V$;

(ii) if $\kappa_{\sigma}=0$, then $\mathcal{V}$ is equal to $\mathcal{V}^{\prime}$, the codimension 1 subspace of $V$ with basis $e_{j}^{t}\left(t \in[1, \sigma], j \in\left[1, p_{t}\right]\right), f_{j}^{t}\left(t \in[1, \sigma], j \in\left[2, p_{t}\right]\right), \epsilon-f_{1}^{t}(t \in[1, \sigma])$ (note that this subspace contains $\left.z_{1}\right)$.

For $t \in[1, \sigma]$ we have

(iii) $E_{j}^{t}=e_{j}^{t}$ if $j \in\left[1, p_{t}\right], E_{p_{t}+1}^{t}=\epsilon-f_{1}^{t}, E_{p_{t}+a}^{t}=2 z_{a-1}+2 z_{a}-f_{a}^{t}$ if $a \geq 2$.

Define a linear map $\phi: V \rightarrow \mathbf{k}$ by $e_{j}^{t} \mapsto 0\left(t \in[1, \sigma], j \in\left[1, p_{t}\right]\right), f_{j}^{t} \mapsto 0(t \in$ $\left.[1, \sigma], j \in\left[2, p_{t}\right]\right), f_{1}^{t} \mapsto 1(t \in[1, \sigma]), \epsilon \mapsto 1$. Then $\phi\left(E_{j}^{t}\right)=0$ if $t \in[1, \sigma], j \in\left[1, p_{t}\right]$, $\phi\left(E_{p_{t}+1}^{t}\right)=0, \phi\left(E_{p_{t}+a}^{t}\right)=0$ if $t \in[1, \sigma], a \geq 3, \phi\left(E_{p_{t}+2}^{t}\right)=2 \phi\left(z_{1}\right)=2 \kappa_{\sigma}$ if $t \in[1, \sigma]$. If $\kappa_{\sigma}=0$, the last expression is 0 so that $\mathcal{V} \subset \operatorname{ker} \phi$ and $\mathcal{V} \neq V$.

In any case from (iii) we see that $e_{j}^{t} \in \mathcal{V}$ for any $t \in[1, \sigma], j \in\left[1, p_{t}\right]$ and $\epsilon-f_{1}^{t} \in \mathcal{V}$ for any $t \in[1, \sigma]$. We have $-\sum_{t \in[1, \sigma]}(-1)^{t}\left(\epsilon-f_{1}^{t}\right)=\kappa_{\sigma} \epsilon-z_{1} \in \mathcal{V}$. For $j \geq 2$ we have (using (iii))

$$
-\sum_{t \in[1, \sigma]}(-1)^{t}\left(2 z_{j-1}+2 z_{j}-f_{j}^{t}\right)=\kappa_{\sigma}\left(2 z_{j-1}+2 z_{j}\right)-z_{j} \in \mathcal{V} .
$$

Thus if $\kappa_{\sigma}=0$ we have $z_{j} \in \mathcal{V}$ for all $j \geq 1$. Hence $f_{j}^{t} \in \mathcal{V}$ for all $t \in[1, \sigma], j \geq 2$; hence $\mathcal{V}^{\prime} \subset \mathcal{V}$. Since $\operatorname{codim}_{V} \mathcal{V}^{\prime}=1$ and $\operatorname{codim}_{V} \mathcal{V} \geq 2$, it follows that $\mathcal{V}=\mathcal{V}^{\prime}$. Now assume that $\kappa_{\sigma}=1$. For $j \geq 1$ we have $2 z_{j}+z_{j+1} \in \mathcal{V}$; hence

$$
z_{j}=2^{-1}\left(2 z_{j}+z_{j+1}\right)-2^{-2}\left(2 z_{j+1}+z_{j+2}\right)+2^{-3}\left(2 z_{j+2}+z_{j+3}\right)-\cdots \in \mathcal{V} .
$$

It follows that $f_{j}^{t} \in \mathcal{V}$ for $t \in[1, \sigma], j \geq 2$. We have $\epsilon-z_{1} \in \mathcal{V}$; hence $\epsilon \in \mathcal{V}$ and $f_{1}^{t} \in \mathcal{V}$ for $t \in[1, \sigma]$. We see that $\mathcal{V}=V$. This proves (i) and (ii). 
2.10. In the setup of $\S 2.7$, we assume that $p \neq 2$. Recall that $\psi:[1, \sigma] \rightarrow\{-1,0,1\}$ is defined in $\S 1.6$. For $t \in[1, \sigma], j \geq 1$ we define $\tilde{E}_{j}^{t} \in V$ by

$$
\tilde{E}_{j}^{t}=E_{j}^{t}-2 \sum_{x \in[1, t-1]}(-1)^{x} E_{p_{x}-p_{t}+j}^{x}-2 \sum_{x \in[1, t-1]}(-1)^{x} E_{p_{x}-p_{t}+j-1}^{x}
$$

if $\psi(t)=1$ (here the last $E_{p_{x}-p_{t}+j-1}^{x}$ is defined since $p_{x}-p_{t}+j-1 \geq 0$ if $\left.p_{x}>p_{t}, j \geq 1\right)$

$$
\tilde{E}_{j}^{t}=E_{j}^{t}-E_{j}^{t-1}
$$

if $t$ is odd and $\psi(t)=0$ (in this case we necessarily have $t>1$; hence $E_{j}^{t-1}$ is defined);

$$
\tilde{E}_{j}^{t}=E_{j}^{t}+\sum_{x \in[1, t-1]}(-1)^{x} E_{p_{x}-p_{t}+j}^{x}-\sum_{v \geq 1}(-2)^{-v+1} \sum_{x \in[1, t-1]}(-1)^{x} E_{p_{x}-p_{t}+v+j-1}^{x}
$$

if $t$ is even. We show:

$$
\text { if } t \in[1, \sigma], j \geq 2 p_{t}+\psi(t)+1 \text {, then } \tilde{E}_{j}^{t}=0 \text {. }
$$

Case 1. Assume that $\psi(t)=1$. In this case we have $j \geq 2 p_{t}+2$. Hence $j \geq p_{t}+3$ and for any $x \in[1, t-1]$ we have $p_{x}-p_{t}+j-1 \geq p_{x}+2$. Thus

$$
\begin{aligned}
& \tilde{E}_{j}^{t}=\left(2 z_{j-p_{t}-1}+2 z_{j-p_{t}}-f_{j-p_{t}}^{t}\right)-2 \sum_{x \in[1, t-1]}(-1)^{x}\left(2 z_{j-p_{t}-1}+2 z_{j-p_{t}}-f_{j-p_{t}}^{x}\right) \\
& -2 \sum_{x \in[1, t-1]}(-1)^{x}\left(2 z_{j-p_{t}-2}+2 z_{j-p_{t}-1}-f_{j-p_{t}-1}^{x}\right) \\
& =\left(2 z_{j-p_{t}-1}+2 z_{j-p_{t}}-f_{j-p_{t}}^{t}\right)+2 \sum_{x \in[1, t-1]}(-1)^{x} f_{j-p_{t}}^{x}+2 \sum_{x \in[1, t-1]}(-1)^{x} f_{j-p_{t}-1}^{x} \\
& =\left(2 z_{j-p_{t}-1}+2 z_{j-p_{t}}-f_{j-p_{t}}^{t}\right)-2 z_{j-p_{t}}-2 \sum_{x \in[t, \sigma]}(-1)^{x} f_{j-p_{t}}^{x} \\
& -2 z_{j-p_{t}-1}-2 \sum_{x \in[t, \sigma]}(-1)^{x} f_{j-p_{t}-1}^{x} \\
& =-f_{j-p_{t}}^{t}-2 \sum_{x \in[t, \sigma]}(-1)^{x} f_{j-p_{t}}^{x}-2 \sum_{x \in[t, \sigma]}(-1)^{x} f_{j-p_{t}-1}^{x} .
\end{aligned}
$$

This is zero since $j-p_{t} \geq p_{t}+1$ and for any $x \in[t, \sigma]$ we have $j-p_{t}-1 \geq p_{x}+1$. 
Case 2. Assume that $t$ is odd and $\psi(t)=0$. In this case we have $t>1$ and $p_{t}=p_{t-1}$. We have $j \geq 2 p_{t}+1$. Hence $j \geq p_{t}+2=p_{t-1}+2$. Thus

$$
\begin{aligned}
\tilde{E}_{j}^{t} & =\left(2 z_{j-p_{t}-1}+2 z_{j-p_{t}}-f_{j-p_{t}}^{t}\right)-\left(2 z_{j-p_{t-1}-1}+2 z_{j-p_{t-1}}-f_{j-p_{t-1}}^{t-1}\right) \\
& =-f_{j-p_{t}}^{t}+f_{j-p_{t-1}}^{t-1} .
\end{aligned}
$$

This is zero since $j-p_{t} \geq p_{t}+1=p_{t-1}+1$.

Case 3. Assume that $\psi(t)=-1$ and $p_{t}>1$. In this case we have $j \geq 2 p_{t}$; hence $j \geq p_{t}+2$ and $p_{x}-p_{t}+v+j-1 \geq p_{x}+2$ (if $x \in[1, t-1], v \geq 1$ ). Thus

$$
\begin{aligned}
\tilde{E}_{j}^{t}= & \left(2 z_{j-p_{t}-1}+2 z_{j-p_{t}}-f_{j-p_{t}}^{t}\right)+\sum_{x \in[1, t-1]}(-1)^{x}\left(2 z_{-p_{t}+j-1}+2 z_{-p_{t}+j}-f_{-p_{t}+j}^{x}\right) \\
& -\sum_{v \geq 2}(-2)^{-v+1} \sum_{x \in[1, t-1]}(-1)^{x}\left(2 z_{-p_{t}+v+j-2}+2 z_{-p_{t}+v+j-1}-f_{-p_{t}+v+j-1}^{x}\right) \\
= & \left(2 z_{j-p_{t}-1}+2 z_{j-p_{t}}-f_{j-p_{t}}^{t}\right)-\left(2 z_{-p_{t}+j-1}+2 z_{-p_{t}+j}\right)-\sum_{x \in[1, t-1]}(-1)^{x} f_{-p_{t}+j}^{x} \\
& +\sum_{v \geq 2}(-2)^{-v+1}\left(2 z_{-p_{t}+v+j-2}+2 z_{-p_{t}+v+j-1}\right) \\
& +\sum_{v \geq 2}(-2)^{-v+1} \sum_{x \in[1, t-1]}(-1)^{x} f_{-p_{t}+v+j-1}^{x} \\
= & \left(2 z_{j-p_{t}-1}+2 z_{j-p_{t}}-f_{j-p_{t}}^{t}\right) \\
& -\left(2 z_{-p_{t}+j-1}+2 z_{-p_{t}+j}\right)+\sum_{v \geq 2}(-2)^{-v+1}\left(2 z_{-p_{t}+v+j-2}+2 z_{-p_{t}+v+j-1}\right) \\
& +z_{-p_{t}+j}+\sum_{x \in[t, \sigma]}(-1)^{x} f_{-p_{t}+j}^{x} \\
& -\sum_{v \geq 2}(-2)^{-v+1} z_{-p_{t}+v+j-1}-\sum_{v \geq 2}(-2)^{-v+1} \sum_{x \in[t, \sigma]}(-1)^{x} f_{-p_{t}+v+j-1}^{x} \\
= & z_{-} p_{t}+j+\sum_{v \geq 2}(-2)^{-v+1}\left(2 z_{-p_{t}+v+j-2}+z_{-p_{t}+v+j-1}\right) \\
& +\sum_{x \in[t+1, \sigma]}(-1)^{x} f_{-p_{t}+j}^{x}-\sum_{v \geq 2}(-2)^{-v+1} \sum_{x \in[t, \sigma]}(-1)^{x} f_{-p_{t}+v+j-1}^{x} \\
= & \sum_{x \in[t+1, \sigma]}(-1)^{x} f_{-p_{t}+j}^{x}-\sum_{v \geq 2}(-2)^{-v+1} \sum_{x \in[t, \sigma]}(-1)^{x} f_{-p_{t}+v+j-1}^{x} .
\end{aligned}
$$

This is zero: for $v \geq 2$ and $x$ in the second sum we have $-p_{t}+v+j-1 \geq p_{x}+1$ (since $j \geq 2 p_{t} \geq p_{t}+p_{x}$ ); for $x$ in the first sum we have $-p_{t}+j \geq p_{x}+1$ (since $\left.j \geq 2 p_{t}>p_{t}+p_{x}\right)$.

Case 4. Assume that $\psi(t)=-1$ and $p_{t}=1$. Since for any $x \in[t+1, \sigma]$ we have $p_{x}<p_{t}$, hence $p_{x}=0$, we see that $[t+1, \sigma]=\emptyset$; hence $t=\sigma$ is even. We can assume that $j=2 p_{t}=2$. We have 


$$
\begin{aligned}
\tilde{E}_{j}^{t}= & E_{2}^{\sigma}+\sum_{x \in[1, \sigma-1]}(-1)^{x} E_{p_{x}+v}^{x}-\sum_{v \geq 1}(-2)^{-v+1} \sum_{x \in[1, \sigma-1]}(-1)^{x} E_{p_{x}+v}^{x} \\
= & \epsilon-f_{1}^{\sigma}+\sum_{x \in[1, \sigma-1]}(-1)^{x}\left(\epsilon-f_{1}^{x}\right) \\
& -\sum_{v \geq 2}(-2)^{-v+1} \sum_{x \in[1, \sigma-1]}(-1)^{x}\left(2 z_{v-1}+2 z_{v}-f_{v}^{x}\right) \\
= & z_{1}-\sum_{v \geq 2}(-2)^{-v+1} \sum_{x \in[1, \sigma-1]}(-1)^{x}\left(2 z_{v-1}+2 z_{v}-f_{v}^{x}\right) \\
= & z_{1}-\sum_{v \geq 2}(-2)^{-v+1}\left(-2 z_{v-1}-2 z_{v}\right)+\sum_{v \geq 2}(-2)^{-v+1} z_{v} \\
= & z_{1}+\sum_{v \geq 2}(-2)^{-v+1}\left(2 z_{v-1}+z_{v}\right)=0 .
\end{aligned}
$$

(We have used that $f_{2}^{\sigma}=0$.)

Case 5. Assume that $t$ is even and $\psi(t)=0$. In this case we can have $j \geq 2 p_{t}+1$; hence $j \geq p_{t}+2$ and $p_{x}-p_{t}+v+j-1 \geq p_{x}+2$ (if $x \in[1, t-1], v \geq 1$ ). By the same computation as in Case 3 we have

$$
\tilde{E}_{j}^{t}=\sum_{x \in[t+1, \sigma]}(-1)^{x} f_{-p_{t}+j}^{x}-\sum_{v \geq 2}(-2)^{-v+1} \sum_{x \in[t, \sigma]}(-1)^{x} f_{-p_{t}+v+j-1}^{x} .
$$

This is zero: for $v \geq 2$ and $x$ in the second sum we have $-p_{t}+v+j-1 \geq p_{x}+1$ (since $j \geq 2 p_{t}+1 \geq p_{t}+p_{x}$ ); for $x$ in the first sum we have $-p_{t}+j \geq p_{x}+1$ (since $\left.j \geq 2 p_{t}+1 \geq p_{t}+p_{x}+1\right)$.

Note that if $\kappa_{\sigma}=0$ we have

$$
N \Xi=-2 \sum_{x \in[1, \sigma]}(-1)^{x}\left(\epsilon-f_{1}^{x}\right)+2 z_{1}+2 z_{2}-2 z_{2}=0 .
$$

We show:

(c) if $\kappa_{\sigma}=1$, the elements $\tilde{E}_{j}^{t}\left(t \in[1, \sigma], j \in\left[1,2 p_{t}+\psi(t)\right]\right)$ form a basis of $V$; if $\kappa_{\sigma}=0$, the elements $\tilde{E}_{j}^{t}\left(t \in[1, \sigma], j \in\left[1,2 p_{t}+\psi(t)\right]\right)$ and $\Xi$ form a basis of $V$. Since $\tilde{E}_{j}^{t}$ is equal to $E_{j}^{t}$ plus a linear combination of elements $E_{j^{\prime}}^{x}$ with $x \in[1, t-1]$ and $E_{j}^{t}=0$ for large $j$ we see that the subspace of V spanned by $\tilde{E}_{j}^{t}(t \in[1, \sigma], j \geq 1)$ coincides with the subspace of $\mathrm{V}$ spanned by $E_{j}^{t}(t \in[1, \sigma], j \geq 1)$, that is with $\mathcal{V}$ (see $\S 2.9$ ). Using $\S 2.9$ we see that if $\kappa_{\sigma}=1$, this subspace is equal to $V$ and that if $\kappa_{\sigma}=0$, this subspace together with $\Xi$ spans $V$. Using this and (b) we see that if $\kappa_{\sigma}=1$, the elements $\tilde{E}_{j}^{t}\left(t \in[1, \sigma], j \in\left[1,2 p_{t}+\psi(t)\right]\right)$ span $V$; if $\kappa_{\sigma}=0$, the elements $\tilde{E}_{j}^{t}\left(t \in[1, \sigma], j \in\left[1,2 p_{t}+\psi(t)\right]\right)$ and $\Xi$ span $V$. It is then enough to show that $\sum_{t \in[1, \sigma]}\left(2 p_{t}+\psi(t)\right)+\left(1-\kappa_{\sigma}\right)=2 n+1$. This follows from 1.6(b).

Using (a),(b) we see that the action of $N$ on the elements in the basis of $V$ described in (c) is as follows:

$$
\tilde{E}_{1}^{t} \mapsto \tilde{E}_{2}^{t} \mapsto \ldots \mapsto \tilde{E}_{2 p_{t}+\psi(t)}^{t} \mapsto 0(t \in[1, \sigma]) \text { and if } \kappa_{\sigma}=0, \Xi \mapsto 0 .
$$

Thus the Jordan blocks of $N: V \rightarrow V$ have sizes $2 p_{1}+\psi(1), 2 p_{2}+\psi(2), \ldots, 2 p_{\sigma}+$ $\psi(\sigma)$ (and 1, if $\kappa_{\sigma}=0$ ). 
2.11. Assume now that $\kappa=1, Q \neq 0, \mathbf{n} \geq 5$. Let $U$ be a codimension 1 subspace of $V$ such that $\left.Q\right|_{U}$ is a nondegenerate quadratic form. Then $U$ together with the restriction of $($,$) and Q$ is as in $\S 1.3$ (with $U$ instead of $V$ ). Define $\mathcal{F}_{0}$ in terms of $U$ in the same way as $\mathcal{F}$ was defined in terms of $V$. We define a map $\iota: \mathcal{F}_{0} \rightarrow \mathcal{F}$ by $\left(0=U_{0} \subset U_{1} \subset \ldots \subset U_{2 n}=U\right) \mapsto\left(0=V_{0} \subset V_{1} \subset \ldots \subset V_{2 n+1}=V\right)$, where $V_{i}=U_{i}$ for $i \in[1, n]$ (note that the $V_{i}$ are then uniquely determined for $i \in[n+1,2 n+1]$. This is an imbedding. If $U_{*} \in \mathcal{F}_{0}, U_{*}^{\prime} \in \mathcal{F}_{0}$, then $a_{U_{*}, U_{*}^{\prime}} \in W$ is defined as in $\S 1.4$ (with $U$ instead of $V$ ). Let $V_{*}=\iota\left(U_{*}\right), V_{*}^{\prime}=\iota\left(U_{*}^{\prime}\right)$. Then $a_{V_{*}, V_{*}^{\prime}} \in W$ is defined as in $\S 1.4$. From the definitions we see that $a_{U_{*}, U_{*}^{\prime}}=a_{V_{*}, V_{*}^{\prime}}$.

2.12. Assume that $\kappa=1, Q \neq 0, \mathbf{n} \geq 5$. Let $p_{*}=\left(p_{1} \geq p_{2} \geq \cdots \geq p_{\sigma}\right) \in \mathcal{P}_{n}^{+}$. Let $w \in W^{\prime}$ and $u_{w}, N$ be as in $\S 2.6$. Let $U$ be the subspace of $V$ spanned by $e_{i}, e_{i}^{\prime}(i \in[1, n])$ if $p=2$ and let $U=(\mathbf{k} \Xi)^{\perp}$ if $p \neq 2$. Then $\operatorname{dim} U=2 n$ and $\left.Q\right|_{U}$ is a nondegenerate quadratic form with associated bilinear form $\left.()\right|_{U$,$} . (If p \neq 2$ we use that $Q(\Xi)=1$.) Note that $U$ is $u_{w}$-stable and $u_{w}$ is a unipotent isometry of $U$. (If $p \neq 2$ we use that $N \Xi=0$.) For $i \in[1, n]$ we have $e_{i} \in U$; hence $\mathfrak{V}_{i} \subset U$. Now $\iota: \mathcal{F}_{0} \rightarrow \mathcal{F}$ is defined as in $\S 2.11$. Define $U_{*}=\left(0=U_{0} \subset U_{1} \subset\right.$ $\left.\ldots \subset U_{2 n}=U\right) \in \mathcal{F}_{0}$, where $U_{i}=\mathfrak{V}_{i}(i \in[1, n])$. Let $U_{*}^{\prime}=u_{w}^{-1} U_{*} \in \mathcal{F}_{0}$. Let $V_{*}=\iota\left(U_{*}\right) \in \mathcal{F}, V_{*}^{\prime}=\iota\left(U_{*}^{\prime}\right)$. Then $V_{*}=\left(0=V_{0} \subset V_{1} \subset \ldots \subset V_{2 n+1}=V\right)$, where $V_{i}=\mathfrak{V}_{i}$ for $i \in[1, n]$ and $V_{*}^{\prime}=u_{w}^{-1}\left(V_{*}\right)$. By 2.4(b) we have $a_{V_{*}, V_{*}^{\prime}}=w_{p_{*}}^{-1} \in W$. By $\S 2.11$ we have $a_{U_{*}, U_{*}^{\prime}}=a_{V_{*}, V_{*}^{\prime}}$. It follows that $a_{U_{*}, U_{*}}=w_{p_{*}}^{-1} \in W$. Since $w_{p_{*}} \in W^{\prime}$ we see that $\left.u_{w}\right|_{U}$ automatically belongs to the identity component of the isometry group of $U$. If $p \neq 2$ the Jordan blocks of $N: U \rightarrow U$ have sizes $2 p_{1}+\psi(1), 2 p_{2}+\psi(2), \ldots, 2 p_{\sigma}+\psi(\sigma)$; indeed, by $\S 2.10$, these are the sizes of the Jordan blocks of $N: V / \mathbf{k} \Xi \rightarrow V / \mathbf{k} \Xi$ which can be identified with $N: U \rightarrow U$ since the direct sum decomposition $V=U \oplus \mathbf{k} \Xi$ is $N$-stable. If $p=2$ the Jordan blocks of $N: U \rightarrow U$ have sizes $2 p_{1}, 2 p_{2}, \ldots, 2 p_{\sigma}$; indeed, by $\S 2.8$, these are the sizes of the Jordan blocks of $N: V / \mathbf{k} e_{0} \rightarrow V / \mathbf{k} e_{0}$ which can be identified with $N: U \rightarrow U$ since the direct sum decomposition $V=U \oplus \mathbf{k} e_{0}$ is $N$-stable.

\section{ISOMETRY GROUPS}

Proposition 3.1. Let $V$ be a k-vector space of finite dimension. Let $g \in G L(V)$ be a unipotent element with Jordan blocks of sizes $n_{1} \geq n_{2} \geq \cdots \geq n_{u} \geq 1$. We set $n_{i}=0$ for $i>u$. Let $m_{1} \geq m_{2} \geq \cdots \geq m_{f} \geq 1$ be integers such that $m_{1}+m_{2}+\cdots+m_{f}=\operatorname{dim} V$. Assume that there exist vectors $x_{1}, x_{2}, \ldots, x_{f}$ in $V$ such that the vectors $g^{i} x_{r}\left(r \in[1, f], i \in\left[0, m_{r}-1\right]\right)$ form a basis of $V$. We set $m_{i}=0$ for $i>f$. For any $c \geq 1$ we have

$$
m_{1}+m_{2}+\cdots+m_{c} \leq n_{1}+n_{2}+\cdots+n_{c} .
$$

In particular we have $u \leq f$.

We set $N=g-1 \in \operatorname{End}(V)$. For $r \in[1, f]$ and $i \in\left[0, m_{r}-1\right]$ we set $v_{r, i}=N^{i} x_{r}$. Note that $\left(v_{r, i}\right)_{r \in[1, f], i \in\left[0, m_{r}-1\right]}$ is a basis of $V$. Note that for any $k \geq 0$, the subspace $N^{k}(V)$ contains the vectors $v_{r, i}\left(r \in[1, f], i \in\left[0, m_{r}-1\right], i \geq k\right)$. Hence

$$
\operatorname{dim} N^{k} V \geq \sum_{r \geq 1} \max \left(m_{r}-k, 0\right) .
$$

By assumption we can find a basis $\left(v_{r, i}^{\prime}\right)_{r \in[1, u], i \in\left[0, n_{r}-1\right]}$ of $V$ such that for any $r \in[1, u]$ we have $v_{r, i}^{\prime}=N v_{r, i-1}^{\prime}$ if $i \in\left[1, n_{r}-1\right], N v_{r, n_{r}-1}^{\prime}=0$. Then for any 
$k \geq 0$ the subspace $N^{k} V$ is spanned by $\left\{v_{r, i}^{\prime} ; r \in[1, u], i \in\left[0, n_{r}-1\right], i \geq k\right\}$. Hence $\operatorname{dim} N^{k}(V)=\sum_{r \geq 1} \max \left(n_{r}-k, 0\right)$. Thus for any $k \geq 0$ we have

$$
\sum_{r \geq 1} \max \left(n_{r}-k, 0\right) \geq \sum_{r \geq 1} \max \left(m_{r}-k, 0\right) .
$$

We show (a) by induction on $c$. Assume that $m_{1}>n_{1}$. The left hand side of (c) with $k=n_{1}$ is $\sum_{r \geq 1} \max \left(n_{r}-n_{1}, 0\right)=0$; the right hand side is $m_{1}-n_{1}$ $+\sum_{r \geq 2} \max \left(m_{r}-k, 0\right) \geq m_{1}-n_{1}>0$; thus we have $0 \geq m_{1}-n_{1}>0$, a contradiction. Thus (a) holds for $c=1$. Assume now that $c \geq 2$ and that (a) holds when $c$ is replaced by $c-1$. Assume that $m_{1}+\cdots+m_{c}>n_{1}+\cdots+n_{c}$. Then

$$
m_{c}-n_{c}>\left(n_{1}+\cdots+n_{c-1}\right)-\left(m_{1}+\cdots+m_{c-1}\right) \geq 0 .
$$

Hence

$$
\max \left(m_{c}-n_{c}, 0\right)>\left(n_{1}+\cdots+n_{c-1}\right)-\left(m_{1}+\cdots+m_{c-1}\right) .
$$

The left hand side of (c) with $k=n_{c}$ is

$$
\sum_{r \geq 1} \max \left(n_{r}-n_{c}, 0\right)=\sum_{r \in[1, c-1]}\left(n_{r}-n_{c}\right)
$$

the right hand side is

$$
\begin{aligned}
& \sum_{r \geq 1} \max \left(m_{r}-n_{c}, 0\right) \geq \sum_{r \in[1, c-1]} \max \left(m_{r}-n_{c}, 0\right)+\left(m_{c}-n_{c}\right) \\
& >\sum_{r \in[1, c-1]} \max \left(m_{r}-n_{c}, 0\right)+\left(n_{1}+\cdots+n_{c-1}\right)-\left(m_{1}+\cdots+m_{c-1}\right) \\
& \geq \sum_{r \in[1, c-1]}\left(m_{r}-n_{c}\right)+\left(n_{1}+\cdots+n_{c-1}\right)-\left(m_{1}+\cdots+m_{c-1}\right) \\
& =\left(n_{1}+\cdots+n_{c-1}\right)-(c-1) n_{c} .
\end{aligned}
$$

Thus (c) implies $\sum_{r \in[1, c-1]}\left(n_{r}-n_{c}\right)>\left(n_{1}+\cdots+n_{c-1}\right)-(c-1) n_{c}$. This is a contradiction. We see that $m_{1}+\cdots+m_{c} \leq n_{1}+\cdots+n_{c}$. This yields the induction step. This proves (a).

We have $n_{1}+n_{2}+\cdots+n_{u}=\operatorname{dim} V=m_{1}+m_{2}+\cdots+m_{f} \leq n_{1}+n_{2}+\cdots+n_{f}$. Hence $n_{1}+n_{2}+\cdots+n_{u} \leq n_{1}+n_{2}+\cdots+n_{f}$. If $u>f$ we deduce $n_{f+1}+\cdots+n_{u} \leq 0$ hence $n_{f+1}=\cdots=n_{u}=0$, which is absurd. Thus $u \leq f$. The proposition is proved.

3.2. In the remainder of this section $V, \mathbf{n}, n,(), Q,, \mathcal{F}, \kappa, I s(V), G$ are as in $\S 1.3$. For any sequence $v_{1}, v_{2}, \ldots, v_{s}$ in $V$ let $S\left(v_{1}, v_{2}, \ldots, v_{s}\right)$ be the subspace of $V$ spanned by $v_{1}, v_{2}, \ldots, v_{s}$.

Let $p_{*}=\left(p_{1} \geq p_{2} \geq \cdots \geq p_{\sigma}\right) \in \mathcal{P}_{n}$. Let $\left(V_{*}, V_{*}^{\prime}\right) \in \mathcal{F} \times \mathcal{F}$ be such that $a_{V_{*}, V_{*}^{\prime}}=w_{p_{*}}$ (see $\left.\S 1.6\right)$. From the definitions we see that for any $r \in[1, \sigma]$ we have

(a) $\operatorname{dim}\left(V_{p_{<r}+i}^{\prime} \cap V_{p_{<r}+i}\right)=p_{<r}+i-r, \quad \operatorname{dim}\left(V_{p_{<r}+i}^{\prime} \cap V_{p_{<r}+i+1}\right)=p_{<r}+i-r+1$ if $i \in\left[1, p_{r}-1\right]$;

$$
\operatorname{dim}\left(V_{p_{\leq r}}^{\prime} \cap V_{\mathbf{n}-p_{<r}-1}\right)=p_{\leq r}-r, \quad \operatorname{dim}\left(V_{p_{\leq r}}^{\prime} \cap V_{\mathbf{n}-p_{<r}}\right)=p_{\leq r}-r+1 .
$$

In this setup we have the following result. 
Proposition 3.3. Let $g \in I s(V)$ be such that $g V_{*}=V_{*}^{\prime}$. There exist vectors $v_{1}, v_{2}, \ldots, v_{\sigma}$ in $V$ (each $v_{i}$ being unique up to multiplication by \pm 1 ) such that, setting $Z_{k}=S\left(v_{k}, g v_{k}, \ldots, g^{p_{k}-1} v_{k}\right)$ for $k \in[1, \sigma]$, the following hold for any $r \in$ $[1, \sigma]:$

(i) $V_{p_{<r}+i}=Z_{1}+\cdots+Z_{r-1}+S\left(v_{r}, g v_{r}, \ldots, g^{i-1} v_{r}\right)$ for $i \in\left[0, p_{r}\right]$;

(ii) $\left(g^{i} v_{t}, v_{r}\right)=0$ for any $1 \leq t<r, i \in\left[-p_{t}, p_{t}-1\right]$;

(iii) $\left(v_{r}, g^{i} v_{r}\right)=0$ for $i \in\left[-p_{r}+1, p_{r}-1\right], Q\left(v_{r}\right)=0$ and $\left(v_{r}, g^{p_{r}} v_{r}\right)=1$;

(iv) the vectors $\left(g^{-p_{t}+i} v_{t}\right)_{t \in[1, r], i \in\left[0,2 p_{t}-1\right]}$ are linearly independent;

(v) setting $E_{r}=S\left(g^{-p_{t}+i} v_{t} ; t \in[1, r], i \in\left[0, p_{t}-1\right]\right)$ we have $V=V_{p_{\leq r}} \oplus E_{r}^{\perp}$.

If $\kappa=1$ there exists a vector $v_{\sigma+1} \in V$ (unique up to multiplication by \pm 1 ) such that

(ii') $\left(g^{i} v_{t}, v_{\sigma+1}\right)=0$ for any $1 \leq t<s+1, i \in\left[-p_{t}, p_{t}-1\right]$;

(iii') $Q\left(v_{\sigma+1}\right)=1$.

Moreover,

(vi) if $\kappa=0$, the vectors $\left(g^{j} v_{t}\right)_{t \in[1, \sigma], j \in\left[-p_{t}, p_{t}-1\right]}$ form a basis of $V$; if $\kappa=1$, the vectors $\left(g^{j} v_{t}\right)_{t \in[1, \sigma], j \in\left[-p_{t}, p_{t}-1\right]}$ together with $v_{\sigma+1}$ form a basis of $V$.

We shall prove the following statement for $u \in[1, \sigma]$.

(a) There exist vectors $v_{1}, v_{2}, \ldots, v_{u}$ in $V$ (each $v_{i}$ being unique up to multiplication by \pm 1 ) such that for any $r \in[1, u]$, (i)-(v) hold.

We can assume that (a) holds when $u$ is replaced by a strictly smaller number in $[1, u]$. (This assumption is empty when $u=1$.) In particular $v_{1}, \ldots, v_{u-1}$ are defined. By assumption we have $V=V_{p_{<u}} \oplus E_{u-1}^{\perp}$; hence $V_{p_{<u}+1} \cap E_{u-1}^{\perp}$ is a line. (We set $E_{0}=0$ so that $E_{0}^{\perp}=V$.) Let $v_{u}$ be a nonzero vector on this line.

We show that (ii) holds for $r \in[1, u]$. It is enough to show this when $r=u$. From the definition we have $V_{p_{<u}+1}=V_{p_{<u}}+\mathbf{k} v_{u}=Z_{1}+\cdots+Z_{u-1}+\mathbf{k} v_{u}$. Since $p_{<u}+1 \leq \mathbf{n} / 2, V_{p_{<u}+1}$ is isotropic. Hence for $t \in[1, u-1]$ we have $\left(Z_{t}, v_{u}\right)=0$; that is, $\left(g^{i} v_{t}, v_{u}\right)=0$ for any $i \in\left[0, p_{t}-1\right]$. From the definition of $v_{u}$ we have $\left(g^{i} v_{t}, v_{u}\right)=0$ for any $t \in[1, u-1]$ and any $i \in\left[-p_{t},-1\right]$. Thus, (ii) holds when $r=u$.

We show that (i) holds for $r \in[1, u]$. It is enough to show (i) when $r=u$ and $i \in\left[0, p_{u}\right]$. We argue by induction on $i$. For $i=0$ the result follows from the induction hypothesis. For $i=1$ the result follows from the definition of $v_{u}$. Assume now that $i \in\left[2, p_{u}\right]$. Let $j=i-1$. By the induction hypothesis we have $V_{p_{<u}+j}=Z_{1}+\cdots+Z_{u-1}+S\left(v_{u}, g v_{u}, \ldots, g^{j-1} v_{u}\right)$; hence $g V_{p_{<u}+j}=g Z_{1}+\cdots+$ $g Z_{u-1}+S\left(g v_{u}, g^{2} v_{u}, \ldots, g^{j} v_{u}\right)$ and the $p_{<u}+j$ vectors

$$
v_{1}, g v_{1}, \ldots, g^{p_{1}-1} v_{1}, \ldots, v_{u-1}, g v_{u-1}, \ldots, g^{p_{u-1}-1} v_{u-1}, v_{u}, g v_{u}, \ldots, g^{j-1} v_{u}
$$

form a basis of $V_{p_{<u}+j}$. Hence the $p_{<u}+j-u$ vectors

$$
g v_{1}, \ldots, g^{p_{1}-1} v_{1}, \ldots, g v_{u-1}, \ldots, g^{p_{u-1}-1} v_{u-1}, g v_{u}, \ldots, g^{j-1} v_{u}
$$

are linearly independent; they are contained in $g V_{p_{<u}+j} \cap V_{p_{<u}+j}$ (of dimension $\left.p_{<u}+j-u\right)$; hence

$$
\begin{aligned}
& S\left(g v_{1}, \ldots, g^{p_{1}-1} v_{1}, \ldots, g v_{u-1}, \ldots, g^{p_{u-1}-1} v_{u-1}, g v_{u}, \ldots, g^{j-1} v_{u}\right) \\
& =g V_{p_{<u}+j} \cap V_{p_{<u}+j} .
\end{aligned}
$$


Since $\operatorname{dim}\left(g V_{p_{<u}+j} \cap V_{p_{<u}+j+1}\right)=p_{<u}+j-u+1$, we see that

$$
\begin{aligned}
& g V_{p_{<u}+j} \cap V_{p_{<u}+j+1} \\
& =S\left(g v_{1}, \ldots, g^{p_{1}-1} v_{1}, \ldots, g v_{u-1}, \ldots, g^{p_{u-1}-1} v_{u-1}, g v_{u}, \ldots, g^{j-1} v_{u}, x\right)
\end{aligned}
$$

for a unique (up to a scalar) $x \in g V_{p_{<u}+j}$ of the form $x=\sum_{r=1}^{u-1} a_{r} g^{p_{r}} v_{r}+a_{u} g^{j} v_{u}$, where $a_{r}, a_{u} \in \mathbf{k}$ are not all 0 .

Assume that $a_{r} \neq 0$ for some $r \in[1, u-1]$; let $r_{0}$ be the smallest such $r$. Note that $V_{p_{<u}+j+1}$ is an isotropic subspace. Since $x \notin V_{p_{<u}+j+1}$ and $v_{r_{0}} \in V_{p_{<u}+j+1}$, we have $\left(x, v_{r_{0}}\right)=0$; hence $\sum_{r=r_{0}}^{u-1} a_{r}\left(g^{p_{r}} v_{r}, v_{r_{0}}\right)+a_{u}\left(g^{j} v_{u}, v_{r_{0}}\right)=0$. By the definition of $v_{u}$ we have $\left(g^{j} v_{u}, v_{r_{0}}\right)=\left(v_{u}, g^{-j} v_{r_{0}}\right)=0$ since $j \in\left[0, p_{u}-1\right] \subset\left[0, p_{r_{0}}-1\right]$. If $r \in\left[r_{0}+1, u-1\right]$ we have $\left(g^{p_{r}} v_{r}, v_{r_{0}}\right)=\left(v_{r}, g^{-p_{r}} v_{r_{0}}\right)=0$ since $-p_{r} \in\left[-p_{r_{0}}, 0\right]$ (we use (ii)). We see that $a_{r_{0}}\left(g^{p_{r_{0}}} v_{r_{0}}, v_{r_{0}}\right)=0$. Using that $\left(g^{p_{r_{0}}} v_{r_{0}}, v_{r_{0}}\right) \neq 0$ (induction hypothesis) we deduce $a_{r_{0}}=0$, a contradiction.

We see that $a_{r}=0$ for any $r \in[1, u-1]$; hence $a_{u} \neq 0$. We can assume that $a_{u}=1$. Thus $x=g^{j} v_{u}$ and

$$
\begin{aligned}
& g V_{p_{<u}+j} \cap V_{p_{<u}+j+1} \\
& =S\left(g v_{1}, \ldots, g^{p_{1}-1} v_{1}, \ldots, g v_{u-1}, \ldots, g^{p_{u-1}-1} v_{u-1}, g v_{u}, \ldots, g^{j-1} v_{u}, g^{j} v_{u}\right) .
\end{aligned}
$$

Now $g V_{p_{<u}+j} \cap V_{p_{<u}+j+1} \not \subset V_{p_{<u}+j}$ (otherwise we would have $g V_{p_{<u}+j} \cap V_{p_{<u}+j+1} \subset$ $g V_{p_{<u}+j} \cap V_{p_{<u}+j}$ and passing to dimensions: $p_{<u}+j-u+1 \leq p_{<u}+j-u$, a contradiction). Since $V_{p_{<u}+j}$ is a hyperplane in $V_{p_{<u}+j+1}$ not containing the subspace $g V_{p_{<u}+j} \cap V_{p_{<u}+j+1}$ of $V_{p_{<u}+j+1}$, we have $V_{p_{<u}+j}+\left(g V_{p_{<u}+j} \cap V_{p_{<u}+j+1}\right)=$ $V_{p_{<u}+j+1}$. It follows that

$$
\begin{aligned}
& V_{p_{<u}+j+1}=S\left(v_{1}, \ldots, g^{p_{1}-1} v_{1}, \ldots, v_{u-1}, \ldots, g^{p_{u-1}-1} v_{u-1}, v_{u}, \ldots, g^{j-1} v_{u}\right) \\
& +S\left(g v_{1}, \ldots, g^{p_{1}-1} v_{1}, \ldots, g v_{u-1}, \ldots, g^{p_{u-1}-1} v_{u-1}, g v_{u}, \ldots, g^{j-1} v_{u}, g^{j} v_{u}\right) \\
& =S\left(v_{1}, \ldots, g^{p_{1}-1} v_{1}, \ldots, v_{u-1}, \ldots, g^{p_{u-1}-1} v_{u-1}, v_{u}, \ldots, g^{j-1} v_{u}, g^{j} v_{u}\right) .
\end{aligned}
$$

Thus (i) holds when $r=u$.

We show that (iii) holds for $r \in[1, u]$. It is enough to show this when $r=$ $u$. Using that $v_{u}, g v_{u}, \ldots, g^{p_{u}-1} v_{u}$ are contained in $V_{p_{\leq u}}$ (see (i)), which is an isotropic subspace ( since $p_{<u} \leq \mathbf{n} / 2$ ), we see that for $i \in\left[0, p_{u}-1\right]$ we have $\left(v_{u}, g^{i} v_{u}\right)=0$; hence also $\left(v_{u}, g^{-i} v_{u}\right)=0$ and moreover $Q\left(v_{u}\right)=0$. We have $\operatorname{dim}\left(g V_{p_{\leq u}} \cap V_{p_{<u}+1}^{\perp}\right)=p_{\leq u}-u$ and $g V_{p_{\leq u}}=S\left(g v_{1}, \ldots, g^{p_{1}} v_{1}, \ldots, g v_{u}, \ldots, g^{p_{u}} v_{u}\right)$. Moreover, using the part of (iii) that is already known, we see that

$$
S\left(g v_{1}, \ldots, g^{p_{1}-1} v_{1}, \ldots, g v_{u}, \ldots, g^{p_{u}-1} v_{u}\right)
$$

(of dimension $p_{\leq u}-u$ ) is contained in $g V_{p_{\leq u}} \cap V_{p_{<u}+1}^{\perp}$, hence is equal to $g V_{p_{\leq u}} \cap$ $V_{p_{<u}+1}^{\perp}$. Hence $g^{p_{u}} v_{u} \notin V_{p_{<u}+1}^{\perp}$; that is,

$$
g^{p_{u}} v_{u} \notin S\left(v, g v_{1}, \ldots, g^{p_{1}-1} v_{1}, \ldots, v_{u-1}, \ldots, g^{p_{u-1}} v_{u-1}, v_{u}\right)^{\perp} .
$$

Since $g^{p_{u}} v_{u} \in S\left(v, g v_{1}, \ldots, g^{p_{1}-1} v_{1}, \ldots, v_{u-1}, \ldots, g^{p_{u-1}} v_{u-1}\right)^{\perp}$ it follows that $\left(g^{p_{u}} v_{u}, v_{u}\right) \neq 0$. Replacing $v_{u}$ by a scalar multiple we can assume that $\left(v_{u}, g^{p_{u}} v_{u}\right)=1$. Thus (iii) holds when $r=u$.

We show that (iv) holds for $r \in[1, u]$. It is enough to show this when $r=u$. Assume that $f=\sum_{r \in[1, u]} \sum_{i \in\left[0,2 p_{r}-1\right]} c_{r, i} g^{-p_{r}+i} v_{r}$ is equal to 0 , where $c_{r, i} \in \mathbf{k}$ are 
not all zero. Let $i_{0}=\min \left\{i: c_{r, i} \neq 0\right.$ for some $\left.r \in[1, u]\right\}$. Let $X=\{r \in[1, u]$ : $\left.c_{r, i_{0}} \neq 0\right\}$. We have $X \neq \emptyset$ and

$$
f=\sum_{r \in X} c_{r, i_{0}} g^{-p_{r}+i_{0}} v_{r}+\sum_{r \in[1, u]} \sum_{i \in\left[i_{0}+1,2 p_{r}-1\right]} c_{r, i} g^{-p_{r}+i} v_{r} .
$$

Let $r_{0}$ be the largest number in $X$. We have

$$
\begin{aligned}
0 & =\left(f, g^{i_{0}} v_{r_{0}}\right)=\sum_{r \in X} c_{r, i_{0}}\left(g^{-p_{r}+i_{0}} v_{r}, g^{i_{0}} v_{r_{0}}\right) \\
& +\sum_{r \in[1, u]} \sum_{i \in\left[i_{0}+1,2 p_{r}-1\right]} c_{r, i}\left(g^{-p_{r}+i} v_{r}, g^{i_{0}} v_{r_{0}}\right) .
\end{aligned}
$$

If $r \in X, r \neq r_{0}$ we have $\left(g^{-p_{r}+i_{0}} v_{r}, g^{i_{0}} v_{r_{0}}\right)=0$ (using (ii) and $r<r_{0}$ ). If $r \in[1, u]$ and $i \in\left[i_{0}+1,2 p_{r}-1\right]$ we have $\left(g^{-p_{r}+i} v_{r}, g^{i_{0}} v_{r_{0}}\right)=0$ (we use (ii),(iii); note that if $r<r_{0}$, we have $-p_{r}+i-i_{0} \in\left[-p_{r}, p_{r}-1\right]$; if $r \geq r_{0}$ we have $\left.p_{r}+i_{0}-i \in\left[-p_{r_{0}}+1, p_{r_{0}}-1\right]\right)$. We see that

$$
0=c_{r_{0}, i_{0}}\left(g^{-p_{r_{0}}+i_{0}} v_{r_{0}}, g^{i_{0}} v_{r_{0}}\right)=c_{r_{0}, i_{0}}\left(g^{-p_{r_{0}}} v_{r_{0}}, v_{r_{0}}\right) \text {. }
$$

Using (iii) we have $\left(g^{-p_{r_{0}}} v_{r_{0}}, v_{r_{0}}\right) \neq 0$; hence $c_{r_{0}, i_{0}}=0$, a contradiction. Thus (iv) holds when $r=u$.

We show that (v) holds for $r \in[1, u]$. It is enough to show this when $r=$ $u$. From (iv) with $r=u$ we see that $\operatorname{dim}\left(E_{u}\right)=p_{\leq u}$. By (i) with $r=u$ we have $E_{u} \subset g^{-p_{t}} V_{p_{\leq u}}$. Since $V_{p_{\leq u}}$ is isotropic we see that $E_{u}$ is isotropic; hence $\operatorname{dim}\left(E_{u}^{\perp}\right)=\mathbf{n}-\operatorname{dim}\left(E_{u}\right)=\mathbf{n}-p_{\leq u}$. Thus $\operatorname{dim}\left(V_{p_{\leq u}}\right)+\operatorname{dim}\left(E_{u}^{\perp}\right)=\operatorname{dim} V$. It is enough to show that $V_{p_{\leq u}} \cap E_{u}^{\perp}=0$. Assume that $f=\sum_{r \in[1, u], i \in\left[1, p_{r}\right]} c_{r, i} g^{p_{r}-i} v_{r}$ belongs to $E_{u}^{\perp}$ and is nonzero. Here $c_{r, i} \in \mathbf{k}$ are not all zero. Let $i_{0}=\min \left\{i: c_{r, i} \neq\right.$ 0 for some $r \in[1, u]\}$. Let $X^{\prime}=\left\{r \in[1, u]: c_{r, i_{0}} \neq 0\right\}$. We have $X^{\prime} \neq \emptyset$ and

$$
f=\sum_{r \in X^{\prime}} c_{r, i_{0}} g^{p_{r}-i_{0}} v_{r}+\sum_{r \in[1, u]} \sum_{i \in\left[i_{0}+1, p_{r}\right]} c_{r, i} g^{p_{r}-i} v_{r} .
$$

Let $r_{0}$ be the smallest number in $X^{\prime}$. We have

$$
\begin{aligned}
& 0=\left(f, g^{-i_{0}} v_{r_{0}}\right)=\sum_{r \in X^{\prime}} c_{r, i_{0}}\left(g^{p_{r}-i_{0}} v_{r}, g^{-i_{0}} v_{r_{0}}\right) \\
& +\sum_{r \in[1, u]} \sum_{i \in\left[i_{0}+1, p_{r}\right]} c_{r, i}\left(g^{p_{r}-i} v_{r}, g^{-i_{0}} v_{r_{0}}\right) .
\end{aligned}
$$

If $r \in X^{\prime}, r \neq r_{0}$, we have $\left(g^{p_{r}-i_{0}} v_{r}, g^{-i_{0}} v_{r_{0}}\right)=\left(v_{r}, g^{-p_{r}} v_{r_{0}}\right)=0$ (we use (ii): note that $r \geq r_{0}$; hence $p_{r} \leq p_{r_{0}}$ ). If $r \in[1, u]$ and $i \in\left[i_{0}+1, p_{r}\right]$, we have $\left(g^{p_{r}-i} v_{r}, g^{-i_{0}} v_{r_{0}}\right)=0$ (we use (ii),(iii); note that if $r>r_{0}$ we have $-p_{r}+i-i_{0} \in$ $\left[-p_{r_{0}}, 0\right]$; if $r \leq r_{0}$ we have $p_{r}-i+i_{0} \in\left[0, p_{r}-1\right]$ ). Thus (v) holds when $r=u$. This completes the inductive proof of (a). Taking $u=\sigma$ in (a) we obtain (i)-(v).

Assume that $\kappa=1$. By (v) we have $V=V_{p_{\leq \sigma}} \oplus E_{\sigma}^{\perp}$; hence $V_{p_{\leq \sigma}+1} \cap E_{\sigma}^{\perp}$ is a line. Let $v_{\sigma+1}$ be a nonzero vector on this line. From the definition we have

$$
V_{p_{\leq \sigma}+1}=V_{p_{\leq \sigma}}+S\left(v_{\sigma+1}\right) .
$$

Since $V_{p_{\leq \sigma}}$ is a maximal isotropic subspace of $V$ and $\left(V_{p_{\leq \sigma}}, v_{\sigma+1}\right)=0$ (by (b)) we see that $Q\left(v_{\sigma+1}\right) \neq 0$. Replacing $v_{\sigma+1}$ by a scalar multiple we can assume that (iii') holds. Using $v_{\sigma+1} \in V_{p_{\leq \sigma}+1}=V_{\pi_{\leq \sigma}}^{\perp}$ and (i) we see that $\left(g^{i} v_{t}, v_{\sigma+1}\right)=0$ for 
$t \in[1, \sigma], i \in\left[0, p_{t}-1\right]$. From $v_{\sigma+1} \in E_{\sigma}^{\perp}$ we have $\left(g^{i} v_{t}, v_{\sigma+1}\right)=0$ for $t \in[1, \sigma]$, $i \in\left[-p_{t},-1\right]$. Thus (ii') holds.

If $\kappa=0$, (vi) follows from (iv) with $r=\sigma$. In the rest of the proof we assume that $\kappa=1$. If $p=2$ we denote by $\omega$ the unique vector in $V^{\perp}$ such that $Q(\omega)=1$. Since $V_{p_{\leq \sigma}+1}=V_{p_{\leq \sigma}}^{\perp}$ we have $\omega \in V_{p_{\leq \sigma}+1}$. Clearly, $\omega \in E_{\sigma}^{\perp}$. Hence $\omega \in V_{p_{\leq \sigma}+1} \cap E_{\sigma}^{\perp}$. Thus we have $v_{\sigma+1}=\omega$. Returning to a general $p$ we show that (vi) holds when $\kappa=1$. Assume that

$$
f=\sum_{r \in[1, \sigma], i \in\left[0,2 p_{r}-1\right]} c_{r, i} g^{-p_{r}+i} v_{r}+c_{\sigma+1,0} v_{\sigma+1}
$$

is equal to 0 , where $c_{r, i} \in \mathbf{k}$ are not all zero. If $c_{\sigma+1,0}=0$, then we have a contradiction by (iv). So we can assume that $c_{\sigma+1,0} \neq 0$ or even that $c_{\sigma+1,0}=1$. We have

$$
0=\left(f, v_{\sigma+1}\right)=\sum_{r \in[1, \sigma], i \in\left[0,2 p_{r}-1\right]} c_{r, i}\left(g^{-p_{r}+i} v_{r}, v_{\sigma+1}\right)+\left(v_{\sigma+1}, v_{\sigma+1}\right) .
$$

For $r, i$ in the sum we have $-p_{r} \leq-p_{r}+i \leq p_{r}-1$; hence $\left(g^{-p_{r}+i} v_{r}, v_{\sigma+1}\right)=0$ (see $\left.\left(\mathrm{ii}^{\prime}\right)\right)$ and hence $\left(v_{\sigma+1}, v_{\sigma+1}\right)=0$. If $p \neq 2$ we have $Q\left(v_{\sigma+1}\right) \neq 0$; hence $\left(v_{\sigma+1}, v_{\sigma+1}\right) \neq 0$, a contradiction. Hence we may assume that $p=2$ so that $v_{\sigma+1}=\omega$. The following proof is almost a repetition of that of (iv). We have $\sum_{r \in[1, \sigma], i \in\left[0,2 p_{r}-1\right]} c_{r, i} g^{-p_{r}+i} v_{r}+\omega=0$. Assume that $c_{r, i} \in \mathbf{k}$ are not all zero. Let $i_{0}=\min \left\{i: c_{r, i} \neq 0\right.$ for some $\left.r \in[1, \sigma]\right\}$. Let $X=\left\{r \in[1, \sigma]: c_{r, i_{0}} \neq 0\right\}$. We have $X \neq \emptyset$ and

$$
\sum_{r \in X} c_{r, i_{0}} g^{-p_{r}+i_{0}} v_{r}+\sum_{r \in[1, \sigma], i \in\left[i_{0}+1,2 p_{r}-1\right]} c_{r, i} g^{-p_{r}+i} v_{r}+\omega=0 .
$$

Let $r_{0}$ be the largest number in $X$. We have

$$
\begin{aligned}
& 0=\left(0, g^{i_{0}} v_{r_{0}}\right)=\sum_{r \in X} c_{r, i_{0}}\left(g^{-p_{r}+i_{0}} v_{r}, g^{i_{0}} v_{r_{0}}\right) \\
& +\sum_{r \in[1, \sigma], i \in\left[i_{0}+1,2 p_{r}-1\right]} c_{r, i}\left(g^{-p_{r}+i} v_{r}, g^{i_{0}} v_{r_{0}}\right) .
\end{aligned}
$$

If $r \in X, r \neq r_{0}$ we have $\left(g^{-p_{r}+i_{0}} v_{r}, g^{i_{0}} v_{r_{0}}\right)=0$ (using (ii) and $r<r_{0}$ ). If $r \in[1, \sigma]$ and $i \in\left[i_{0}+1,2 p_{r}-1\right]$ we have $\left(g^{-p_{r}+i} v_{r}, g^{i_{0}} v_{r_{0}}\right)=0$ (we use (ii),(iii): note that if $r<r_{0}$, we have $-p_{r}+i-i_{0} \in\left[-p_{r}, p_{r}-1\right]$; if $r \geq r_{0}$ we have $\left.p_{r}+i_{0}-i \in\left[-p_{r_{0}}+1, p_{r_{0}}-1\right]\right)$. We see that

$$
0=c_{r_{0}, i_{0}}\left(g^{-p_{r_{0}}+i_{0}} v_{r_{0}}, g^{i_{0}} v_{r_{0}}\right)=c_{r_{0}, i_{0}}
$$

(we have used (iii)); hence $c_{r_{0}, i_{0}}=0$, a contradiction. We see that $c_{r, i}=0$ for all $r \in[1, \sigma], i \in\left[0,2 p_{r}-1\right]$. Hence $\omega=0$, a contradiction. This proves (vi). The proposition is proved.

3.4. We preserve the setup of Proposition 3.3. For any $r \in[1, \sigma]$, let $X_{r}$ be the subspace of $V$ spanned by $\left(g^{-p_{r}+i} v_{r}\right)_{i \in\left[0,2 p_{r}-1\right]}$. Let $X_{\sigma+1}$ be 0 (if $\kappa=0$ ) and the subspace spanned by $v_{\sigma+1}$ (if $\kappa=1$ ). From Proposition 3.3(vi) we see that

$$
V=\bigoplus_{r \in[1, \sigma+1]} X_{r}
$$


For $t \in[1, \sigma]$, let $W_{t}=\bigoplus_{r \in[1, t]} X_{r}, W_{t}^{\prime}=\bigoplus_{r \in[t+1, \sigma+1]} X_{r}$. From (a) we see that

$$
V=W_{t} \oplus W_{t}^{\prime} \text {. }
$$

We show:

$$
V=X_{r} \oplus X_{r}^{\perp} \text { if } r \in[1, \sigma] .
$$

Let $f=\sum_{i \in\left[0,2 p_{r}-1\right]} c_{r, i} g^{-p_{r}+i} v_{r}$ be such that $\left(f, g^{j} v_{r}\right)=0$ for any $j \in\left[-p_{r}, p_{r}-1\right]$. Here $c_{r, i} \in \mathbf{k}$. We show that $c_{r, i}=0$ for all $i$. Assume that $c_{r, i} \neq 0$ for some $i \in\left[0,2 p_{r}-1\right]$ and let $i_{0}$ be the smallest $i$ such that $c_{r, i} \neq 0$. Assume first that $i_{0} \in\left[0, p_{r}-1\right]$. We have

$$
\begin{aligned}
0 & =\left(f, g^{i_{0}} v_{r}\right)=\sum_{i \in\left[i_{0}, 2 p_{r}-1\right]} c_{r, i}\left(g^{-p_{r}+i} v_{r}, g^{i_{0}} v_{r}\right) \\
& =c_{r, i_{0}}\left(g^{-p_{r}} v_{r}, v_{r}\right)+\sum_{i \in\left[i_{0}+1,2 p_{r}-1\right]} c_{r, i}\left(g^{-p_{r}+i-i_{0}} v_{r}, v_{r}\right) .
\end{aligned}
$$

In the last sum we have $-p_{r}+1 \leq-p_{r}+i-i_{0} \leq p_{r}-1$; hence the last sum is zero (see Proposition 3.3(iii)). We see that $c_{r, i_{0}}\left(g^{-p_{r}} v_{r}, v_{r}\right)=0$; hence $c_{r, i_{0}}=0$ (see Proposition 3.3(iii)), a contradiction. Thus we have $i_{0} \in\left[p_{r}, 2 p_{r}-1\right]$ so that $f=\sum_{i \in\left[p_{r}, 2 p_{r}-1\right]} c_{r, i} g^{-p_{r}+i} v_{r}$. Let $i_{1}$ be the largest $i$ such that $c_{r, i} \neq 0$. We have $i_{1} \in\left[p_{r}, 2 p_{r}-1\right] ;$ hence $-2 p_{r}+i_{1} \in\left[-p_{r},-1\right]$. We have

$$
\begin{aligned}
0 & =\left(f, g^{-2 p_{r}+i_{1}} v_{r}\right)=\sum_{i \in\left[p_{r}, i_{1}\right]} c_{r, i}\left(g^{-p_{r}+i} v_{r}, g^{-2 p_{r}+i_{1}} v_{r}\right) \\
& =c_{r, i_{1}}\left(g^{p_{r}} v_{r}, v_{r}\right)+\sum_{i \in\left[p_{r}, i_{1}-1\right]} c_{r, i}\left(g^{p_{r}+i-i_{1}} v_{r}, v_{r}\right) .
\end{aligned}
$$

In the last sum we have $-p_{r}+1 \leq p_{r}+i-i_{1} \leq p_{r}-1$; hence the last sum is zero (see Proposition 3.3(iii)). We see that $c_{r, i_{1}}\left(g^{p_{r}} v_{r}, v_{r}\right)=0$; hence $c_{r, i_{1}}=0$, a contradiction. We see that $X_{r} \cap X_{r}^{\perp}=0$. We have $\operatorname{dim} X_{r}^{\perp}+\operatorname{dim} X_{r} \geq \operatorname{dim} V$; hence $V=X_{r} \oplus X_{r}^{\perp}$, as required.

3.5. In the setup of Proposition 3.3 we assume that $g$ is unipotent; we set $N=$ $g-1 \in \operatorname{End}(V)$. We set $p_{\sigma+1}=\kappa / 2$. Note that $\pi_{1} \geq \pi_{2} \geq \cdots \geq \pi_{\sigma} \geq p_{\sigma+1}$. For any $k \geq 0$ we set

$$
\Lambda_{k}=\sum_{r \in[1, \sigma+1]} \max \left(2 p_{r}-k, 0\right)
$$

We show:

(a) For any $k \in \mathbf{N}$ we have $\operatorname{dim} N^{k} V \geq \Lambda_{k}$. Moreover, $\operatorname{dim} N^{0} V=\mathbf{n}=\Lambda_{0}$. The inequality in (a) follows from 3.1(b) using Proposition 3.3(vi). (We apply 3.1(b) with $x_{1}, \ldots, x_{f}$ given by $g^{-p_{1}} v_{1}, \ldots, g^{-p_{\sigma}} v_{\sigma}$ if $\kappa=0$, or by $g^{-p_{1}} v_{1}, \ldots, g^{-p_{\sigma}} v_{\sigma}, v_{\sigma+1}$ if $\kappa=1$.) The equality in (a) follows from $\Lambda_{0}=\sum_{r \in[1, \sigma+1]} 2 p_{r}=\mathbf{n}$.

We now assume that $k>0$ and $d \in[1, \sigma]$ is such that $2 p_{d} \geq k \geq 2 p_{d+1}$. Then $\Lambda_{k}=\sum_{r \in[1, d]}\left(2 p_{r}-k\right)$. We show:

(b) If $\operatorname{dim} N^{k} V=\Lambda_{k}$, then $W_{d}, W_{d}^{\prime}$ are $g$-stable, $W_{d}^{\prime}=W_{d}^{\perp}, g: W_{d} \rightarrow W_{d}$ has exactly d Jordan blocks (each one has size $\geq k$ ) and $N^{k} W_{d}^{\prime}=0$.

For $r \in[1, \sigma]$ let $v_{r}^{\prime}=g^{-p_{r}} v_{r}$. Then $\left(g^{i} v_{r}^{\prime}\right)_{i \in\left[0,2 p_{r}-1\right]}$ is a basis of $X_{r}$; hence $\left(N^{i} v_{r}^{\prime}\right)_{i \in\left[0,2 p_{r}-1\right]}$ is a basis of $X_{r}$. For $r \in[1, d]$ let $Y_{r}$ be the subspace spanned by $N^{i} v_{r}^{\prime}\left(i \in\left[k, 2 p_{r}-1\right]\right)$. Note that $Y_{r} \subset N^{k} X_{r}$. Hence $\bigoplus_{r \in[1, d]} Y_{r} \subset N^{k} W_{d} \subset$ $N^{k} V$. We have $\operatorname{dim} \bigoplus_{r \in[1, d]} Y_{r}=\sum_{r \in[1, d]}\left(2 p_{r}-k\right)=\Lambda_{k}=\operatorname{dim} N^{k} V$. Hence 
$\bigoplus_{r \in[1, d]} Y_{r}=N^{k} W_{d}=N^{k} V$. We have $\bigoplus_{r \in[1, d]} Y_{r} \subset W_{d}$. Hence $N^{k} V \subset W_{d}$. We show that $N W_{d} \subset W_{d}$. Clearly $N$ maps the basis elements $N^{i} v_{r}^{\prime}(r \in[1, d], i \in$ $\left.\left[0,2 p_{r}-2\right]\right)$ into $W_{d}$. So it is enough to show that $N$ maps $N^{2 p_{r}-1} v_{r}^{\prime}(r \in[1, d])$ into $W_{d}$. But $N N^{2 p_{r}-1} v_{r}^{\prime}=N^{2 p_{r}} v_{r}^{\prime}=N^{k} N^{2 p_{r}-k} v_{r}^{\prime} \subset N^{k} V \subset W_{d}$. Thus $N W_{d} \subset W_{d}$. Hence $g W_{d}=W_{d}$ and $g W_{d}^{\perp}=W_{d}^{\perp}$. For $r \in[d+1, \sigma]$ we have $v_{r} \in W_{d}^{\perp}$ by Proposition 3.3(ii). Since $g W_{d}^{\perp}=W_{d}^{\perp}$ we have $g^{j} v_{r} \in W_{d}^{\perp}$ for all $j \in \mathbf{Z}$; hence $X_{r} \subset W_{d}^{\perp}$. Similarly, if $\kappa=1$ we have $v_{\sigma+1} \in W_{d}^{\perp}$ by Proposition 3.3(ii'); hence $X_{\sigma+1} \subset W_{d}^{\perp}$. We see that in any case $W_{d}^{\prime} \subset W_{d}^{\perp}$. Since $W_{d} \oplus W_{d}^{\prime}=V$, it follows that $W_{d}+W_{d}^{\perp}=V$. Since $V^{\perp} \subset W_{d}^{\prime}$ we have $V^{\perp} \cap W_{d}=0$; hence $\operatorname{dim} W_{d}^{\perp}=$ $\operatorname{dim} V-\operatorname{dim} W_{d}$, which, together with $W_{d}+W_{d}^{\perp}=V$, implies $W_{d} \oplus W_{d}^{\perp}=V$ and $W_{d}^{\perp}=W_{d}^{\prime}$. In particular, $W_{d}^{\prime}$ is $g$-stable. Let $\delta$ be the number of Jordan blocks of $N: W_{d} \rightarrow W_{d}$; that is, $\delta=\operatorname{dim}\left(\operatorname{ker} N: W_{d} \rightarrow W_{d}\right)$. We have $\operatorname{dim} W_{d}-\delta=$ $\operatorname{dim} N W_{d} \geq \sum_{r \in[1, d]}\left(2 p_{r}-1\right)=\operatorname{dim} W_{d}-d$. (The inequality follows from 3.1(b) applied to $N: W_{d} \rightarrow W_{d}$.) Hence $\delta \leq d$. From the definition of $\delta$ we see that $\operatorname{dim}\left(\operatorname{ker} N^{k}: W_{d} \rightarrow W_{d}\right) \leq \delta k$. Recall that $\operatorname{dim} N^{k} W_{d}=\sum_{r \in[1, d]}\left(2 p_{r}-k\right)=$ $\operatorname{dim} W_{d}-k d$. Hence $\operatorname{dim}\left(\operatorname{ker} N^{k}: W_{d} \rightarrow W_{d}\right)=\operatorname{dim} W_{d}-\operatorname{dim} N^{k} W_{d}=k d$. Hence $k d \leq \delta k$. Since $k>0$ we deduce $d \leq \delta$. Hence $d=\delta$. Since $\operatorname{dim}\left(\operatorname{ker} N^{k}: W_{d} \rightarrow\right.$ $\left.W_{d}\right)=k d$ we see that each of the $d$ Jordan blocks of $N: W_{d} \rightarrow W_{d}$ has size $\geq k$. Since $N^{k} W=N^{k} V$ and $V=W_{d} \oplus W_{d}^{\prime}$ we see that $N^{k} W_{d}^{\prime}=0$. Hence each Jordan block of $N: W_{d}^{\prime} \rightarrow W_{d}^{\prime}$ has size $\leq k$. This proves (b).

We show:

(c) if $\operatorname{dim} N^{k} V=\Lambda_{k}$ for all $k \geq 0$, then for any $r \in[1, \sigma+1], X_{r}$ is a g-stable subspace of $V$ and for any $r \neq r^{\prime}$ in $[1, \sigma+1]$ we have $\left(X_{r}, X_{r^{\prime}}\right)=0$.

Applying (b) with $k=2 p_{d}$ for $d=1,2, \ldots, \sigma$ we see that each of the subspaces $X_{1} \subset X_{1} \oplus X_{2} \subset \ldots \subset X_{1} \oplus X_{2} \oplus \ldots \oplus X_{\sigma}$ of $V$ is $g$-stable and each of the subspaces $X_{2} \oplus \ldots \oplus X_{\sigma+1} \supset \ldots \supset X_{\sigma} \oplus X_{\sigma+1} \supset X_{\sigma+1}$ of $V$ is $g$-stable. Taking intersections we see that each of the subspaces $X_{1}, X_{2}, \ldots, X_{\sigma+1}$ of $V$ is $g$-stable. The second assertion of (c) also follows from (b).

3.6. We preserve the setup of $\S 3.5$ and we assume that $p \neq 2, Q \neq 0$. For any $k>0$ such that $\operatorname{dim} N^{k} V=\Lambda_{k}$ we show:

(a) if $d \in[1, \sigma]$ is such that $k \in\left[2 p_{d+1}, 2 p_{d}\right]$, then $d$ is even;

(b) $k \neq 2 p_{r}$ for $r \in[1, \sigma]$.

The proof is based on the following known property of a unipotent isometry $T$ : $W \rightarrow W$ of a finite dimensional $\mathbf{k}$-vector space $W$ with a nondegenerate symmetric bilinear form (assuming $p \neq 2$ ): the number of Jordan blocks of $T$ is congruent $\bmod 2$ to $\operatorname{dim} W$.

By 3.5(b), $W_{d}$ is $g$-stable and (, ) is nondegenerate on $W_{d}$. Hence $g: W_{d} \rightarrow W_{d}$ has an even number of Jordan blocks. (Clearly, $\operatorname{dim} W_{d}$ is even.) By 3.5(b), $g$ : $W_{d} \rightarrow W_{d}$ has exactly $d$ Jordan blocks. Hence $d$ is even, proving (a).

Assume now that $k=2 p_{r}$ for some $r \in[1, \sigma]$. If $r$ is odd we have $k \in\left[2 p_{r+1}, 2 p_{r}\right]$; hence by (a), $r$ is even, a contradiction. If $r$ is even we have $r \geq 2$ and $k \in$ $\left[2 p_{r}, 2 p_{r-1}\right]$; hence by (a), $r-1$ is even, a contradiction. This proves (b).

For any $k \geq 0$ we define $\Lambda_{k}^{\prime} \in \mathbf{N}$ by $\Lambda_{k}^{\prime}=\Lambda_{k}+1$ if $k>0, k \in\left[2 p_{d+1}, 2 p_{d}\right]$ for some odd $\bar{d} \in[1, \sigma]$ and $\Lambda_{k}^{\prime}=\Lambda_{k}$ otherwise. In particular, if $k=2 p_{r}$ for some $r \in[1, \sigma]$, then $\Lambda_{k}^{\prime}=\Lambda_{k}+1$. (If $r$ is odd we have $k \in\left[2 p_{r+1}, p_{r}\right]$; hence $\Lambda_{k}^{\prime}=\Lambda_{k}+1$. If $r$ is even we have $r \geq 2$ and $k \in\left[2 p_{r}, 2 p_{r-1}\right]$; hence $\Lambda_{k}^{\prime}=\Lambda_{k}+1$.) From 3.6(a) and 3.5(a) we see: 
(c) $\operatorname{dim} N^{k} V \geq \Lambda_{k}^{\prime}$ for any $k \geq 0$; moreover $\operatorname{dim} N^{0} V=\mathbf{n}=\Lambda_{0}^{\prime}$.

For $r \in[1, \sigma+1]$ we set $\pi_{r}=2 p_{r}+\psi(r)$, where

$\psi(r)=1$ if $r$ is odd, $r \leq \sigma$ and $p_{r-1}>p_{r}$ (convention: $p_{0}=\infty$ );

$\psi(r)=-1$ if $r$ is even and $p_{r}>p_{r+1}\left(\right.$ convention: $\left.p_{\sigma+2}=0\right)$;

$\psi(r)=0$ for all other $r$.

When $r \in[1, \sigma]$ this definition of $\psi(r)$ agrees with that in $\S 1.6$. Note that $\psi(\sigma+1)$ equals -1 if $\kappa_{\sigma}=\kappa=1$ and equals 0 otherwise.

In the remainder of this subsection we assume that

(d) if $\kappa=0$, then $\kappa_{\sigma}=0$.

For any $k \geq 0$ we set $\Lambda_{k}^{\prime \prime}=\sum_{r \in[1, \sigma+1]} \max \left(\pi_{r}-k, 0\right)$. We show:

$$
\Lambda_{k}^{\prime \prime}=\Lambda_{k}^{\prime} \text { for all } k \geq 0 .
$$

Assume first that $2 p_{d}>k>2 p_{d+1}$ for some $d \in[1, \sigma]$. Then the conditions $2 p_{r} \geq k, 2 p_{r}>k, \pi_{r} \geq k, r \leq d$ are equivalent; hence

$$
\Lambda_{k}^{\prime \prime}-\Lambda_{k}=\sum_{r \in[1, \sigma+1] ; 2 p_{r} \geq k}\left(\pi_{r}-k\right)-\sum_{r \in[1, \sigma+1] ; 2 p_{r} \geq k}\left(2 p_{r}-k\right)=\sum_{r \in[1, d]} \psi(r) .
$$

This equals $1=\Lambda_{k}^{\prime}-\Lambda_{k}$ if $d$ is odd and equals $1+\psi(d)=1-1=0=\Lambda_{k}^{\prime}-\Lambda_{k}$ if $d$ is even.

Next we assume that $k=2 p_{d^{\prime}}$ for some $d^{\prime} \in[1, \sigma]$. There is a unique $d \in[1, \sigma]$ such that $2 p_{d^{\prime}}=2 p_{d}>2 p_{d+1}$. The condition that $\pi_{r} \geq k$ is equivalent to $2 p_{r} \geq 2 p_{d}$ (if $\psi(r) \in\{0,1\}$ ) and to $2 p_{r} \geq 2 p_{d}, p_{r} \neq p_{d}$ (if $\psi(r)=-1$ ). Moreover for $r$ such that $p_{r}=p_{d}, r \neq d$ we have $p_{r}=p_{r+1}$; hence $\psi(r) \neq-1$. Thus

$$
\begin{aligned}
& \Lambda_{k}^{\prime \prime}-\Lambda_{k}=\sum_{r \in[1, \sigma+1] ; 2 p_{r} \geq 2 p_{d}}\left(\pi_{r}-2 p_{d}\right)-\sum_{r \in[1, \sigma+1] ; \psi(r)=-1 ; 2 p_{r}=2 p_{d}}\left(2 p_{r}-1-2 p_{d}\right) \\
& -\sum_{r \in[1, \sigma+1] ; 2 p_{r} \geq 2 p_{d}}\left(2 p_{r}-2 p_{d}\right)=\sum_{r \in[1, d]} \psi(r)+\sum_{r \in[1, \sigma+1] ; \psi(r)=-1 ; r=d} 1 .
\end{aligned}
$$

By $\S 1.6$ this equals $(1+\psi(d))+1=(1-1)+1=1=\Lambda_{k}^{\prime}-\Lambda_{k}$ if $d$ is even (so that $\psi(d)=-1$ ) and equals $1+0=\Lambda_{k}^{\prime}-\Lambda_{k}$ if $d$ is odd (so that $\psi(d) \neq-1$ ).

Next we assume that $k \leq 2 p_{\sigma+1}$. Then $k=0$ and $\pi_{r} \geq k, 2 p_{r} \geq k$ for all $r \in[0,1+\sigma]$; hence

$$
\Lambda_{k}^{\prime \prime}-\Lambda_{k}=\sum_{r \in[1, \sigma+1]}\left(\pi_{r}-2 p_{\sigma+1}\right)-\sum_{r \in[1, \sigma+1]}\left(2 p_{r}-2 p_{\sigma+1}\right)=\sum_{r \in[1, \sigma+1]} \psi(r) .
$$

This equals $(1+\psi(\sigma))+\psi(\sigma+1)=(1-1)+0=0=\Lambda_{k}^{\prime}-\Lambda_{k}$ if $\sigma$ is even and equals $1+\psi(\sigma+1)=1-1=0=\Lambda_{k}^{\prime}-\Lambda_{k}$ if $\sigma$ is odd. (We use $\S 1.6$.)

Finally assume that $k>2 p_{1}$. We have $\pi_{r} \leq k, 2 p_{r}<k$ for all $r \in[0,1+\sigma]$; hence $\Lambda_{k}^{\prime \prime}-\Lambda_{k}=0-0=0=\Lambda_{k}^{\prime}-\Lambda_{k}$.

We see that for any $k \geq 0$ we have $\Lambda_{k}^{\prime \prime}-\Lambda_{k}=\Lambda_{k}^{\prime}-\Lambda_{k}$ and (e) follows.

3.7. We prove Proposition 0.6 in the case where $G$ is as in $\S 1.3$ with $\kappa=0, Q=$ $0, p \neq 2$. Let $p_{*}=\left(p_{1} \geq p_{2} \geq \cdots \geq p_{\sigma}\right) \in \mathcal{P}_{n}$. Let $B, B^{\prime}$ be as in $\S 2.4$; define $u_{w}$ in terms of the excellent decomposition 2.2(a) of $w=w_{p_{*}}^{-1}$ as in $\S 2.4$. Let $\gamma_{p_{*}}$ be the $G$-conjugacy class of $u_{w}$. Let $N_{0}=u_{w}^{-1}-1$. By $\S 2.6, N_{0}$ has Jordan blocks of sizes $2 p_{1}, 2 p_{2}, \ldots, 2 p_{\sigma}$. Hence for any $k \geq 0$ we have $\operatorname{dim} N_{0}^{k}=\sum_{r \in[1, \sigma]} \max \left(2 p_{r}-k, 0\right)$. By 2.4(b) we have $\left(B, u_{w} B u_{w}^{-1}\right) \in \mathcal{O}_{w}$. Since $w \in\left(C_{p_{*}}\right)_{\text {min }}$, we have $C_{p_{*}} \dashv \gamma_{p_{*}}$. Now let $\gamma^{\prime} \in \underline{\underline{G}}$ be such that $C_{p_{*}} \dashv \gamma^{\prime}$. Then there exists $g \in \gamma^{\prime}$ such that $g B g^{-1}=$ $u_{w} B u_{w}^{-1}$. Define $V_{*}, V_{*}^{\prime} \in \mathcal{F}$ by $B_{V_{*}}=B, B_{V_{*}^{\prime}}=u_{w} B u_{w}^{-1}$. We have $a_{V_{*}, V_{*}^{\prime}}=w$ 
(since $\left.\left(B, u_{w} B u_{w}^{-1}\right) \in \mathcal{O}_{w}\right)$ and $g V_{*}=V_{*}^{\prime}\left(\right.$ since $\left.g B g^{-1}=u_{w} B u_{w}^{-1}\right)$. By 3.5(a) for any $k \geq 0$ we have $\operatorname{dim} N^{k} V \geq \sum_{r \in[1, \sigma]} \max \left(2 p_{r}-k, 0\right)$; hence $\operatorname{dim} N^{k} V \geq$ $\operatorname{dim} N_{0}^{k} V$. It follows that the conjugacy class of $u_{w}^{-1}$ in $G L(V)$ is contained in the closure of the conjugacy class of $g$ in $G L(V)$. Since $p \neq 2$ it follows that the conjugacy class of $u_{w}^{-1}$ in $G$ is contained in the closure of the conjugacy class of $g$ in $G$. Since $\gamma_{p_{*}}=\gamma_{p_{*}}^{-1}$ we see that $\gamma_{p_{*}}$ is contained in the closure of $\gamma^{\prime}$. We see that property $\Pi_{C_{p_{*}}}$ holds with $\gamma_{C_{p_{*}}}=\gamma_{p_{*}}$. The map $p_{*} \mapsto \gamma_{p_{*}}$ is clearly injective. If $\gamma_{0}$ is a distinguished unipotent class in $G$, then all its Jordan blocks have even sizes (with multiplicity one). Hence $\gamma_{0}$ is of the form $\gamma_{p_{*}}$ for some $p_{*} \in \mathcal{P}_{n}$. This completes the proof of Proposition 0.6 in our case.

3.8. We prove Proposition 0.6 in the case where $G$ is as in $\S 1.3$ with $\kappa=1, Q \neq$ $0, p \neq 2$. Let $p_{*}=\left(p_{1} \geq p_{2} \geq \cdots \geq p_{\sigma}\right) \in \mathcal{P}_{n}$. Let $B, B^{\prime}$ be as in $\S 2.4$; define $u_{w}$ in terms of the excellent decomposition 2.2(a) of $w=w_{p_{*}}^{-1}$ as in $\S 2.4$. Let $\gamma_{p_{*}}$ be the $G$-conjugacy class of $u_{w}$. Let $N_{0}=u_{w}^{-1}-1$. By $\S 2.11, N_{0}$ has Jordan blocks of sizes $2 p_{1}+\psi(1), 2 p_{2}+\psi(2), \ldots, 2 p_{\sigma}+\psi(s)$ (and 1 if $\sigma$ is even). Here $\psi$ is as in $\S 1.6$. Hence for any $k \geq 0$ we have $\operatorname{dim} N_{0}^{k}=\Lambda_{k}^{\prime \prime}$ (notation of $\S 3.6$ ). By 2.4(b) we have $\left(B, u_{w} B u_{w}^{-1}\right) \in \mathcal{O}_{w}$. Since $w \in\left(C_{p_{*}}\right)_{\min }$, we have $C_{p_{*}} \dashv \gamma_{p_{*}}$. Now let $\gamma^{\prime} \in \underline{G}$ be such that $C_{p_{*}} \dashv \gamma^{\prime}$. Then there exists $g \in \gamma^{\prime}$ such that $g B g^{-1}=u_{w} B u_{w}^{-\overline{\overline{1}}}$. Define $V_{*}, V_{*}^{\prime} \in \mathcal{F}$ by $B_{V_{*}}=B, B_{V_{*}^{\prime}}=u_{w} B u_{w}^{-1}$. We have $a_{V_{*}, V_{*}^{\prime}}=w$ (since $\left.\left(B, u_{w} B u_{w}^{-1}\right) \in \mathcal{O}_{w}\right)$ and $g V_{*}=V_{*}^{\prime}$ (since $\left.g B g^{-1}=u_{w} B u_{w}^{-1}\right)$. By 3.6(c),(e) for any $k \geq 0$ we have $\operatorname{dim} N^{k} V \geq \Lambda_{k}^{\prime \prime}$; hence $\operatorname{dim} N^{k} V \geq \operatorname{dim} N_{0}^{k} V$. It follows that the conjugacy class of $u_{w}^{-1}$ in $G L(V)$ is contained in the closure of the conjugacy class of $g$ in $G L(V)$. Since $p \neq 2$ it follows that the conjugacy class of $u_{w}^{-1}$ in $G$ is contained in the closure of the conjugacy class of $g$ in $G$. Since $\gamma_{p_{*}}=\gamma_{p_{*}}^{-1}$ we see that $\gamma_{p_{*}}$ is contained in the closure of $\gamma^{\prime}$. We see that property $\Pi_{C_{p_{*}}}$ holds with $\gamma_{C_{p_{*}}}=\gamma_{p_{*}}$. This completes the proof of Proposition 0.6(i) in our case.

We prove the injectivity in Proposition 0.6(ii). Let $\Pi$ be the set of all sequences $\pi_{1} \geq \pi_{2} \geq \cdots \geq \pi_{\sigma^{\prime}}$ of integers $\geq 1$ such that $\pi_{1}+\pi_{2}+\cdots+\pi_{\sigma^{\prime}}=\mathbf{n}$. We define a $\operatorname{map} \phi: \mathcal{P}_{n} \rightarrow \Pi$ by odd;

(i) $\left(p_{1} \geq p_{2} \geq \cdots \geq p_{\sigma}\right) \mapsto\left(2 p_{1}+\psi(1) \geq 2 p_{2}+\psi(2) \geq \cdots \geq 2 p_{\sigma}+\psi(\sigma)\right)$ if $\sigma$ is

(ii) $\left(p_{1} \geq p_{2} \geq \cdots \geq p_{\sigma}\right) \mapsto\left(2 p_{1}+\psi(1) \geq 2 p_{2}+\psi(2) \geq \cdots \geq 2 p_{\sigma}+\psi(\sigma) \geq 1\right)$ if $\sigma$ is even.

It is enough to show that $\phi$ is injective. We show that $2 p_{i}+\psi(i) \geq 2 p_{i+1}+\psi(i+1)$ for $i \in[1, \sigma-1]$. If $p_{i}>p_{i+1}$, then $2 p_{i} \geq 2 p_{i+1}+2$ and it is enough to show that $\psi(i)-\psi(i+1) \geq-2$; this is clear since $\psi(i) \geq-1,-\psi(i+1) \geq-1$. So we can assume that $p_{i}=p_{i+1}$. If $i$ is even, then $\psi(i+1)=0, \psi(i) \geq 0$; hence $2 p_{i}+\psi(i) \geq 2 p_{i+1}+\psi(i+1)$. If $i$ is odd, then $\psi(i+1) \leq 0, \psi(i)=0$ and again $2 p_{i}+\psi(i) \geq 2 p_{i+1}+\psi(i+1)$. Moreover in case (ii) we have $2 p_{\sigma}+\psi(\sigma) \geq 1$ (since $2 p_{\sigma} \geq 2, \psi(\sigma) \geq-1$ ). We see that $\phi$ is well defined. Assume now that $p_{*}=\left(p_{1} \geq p_{2} \geq \cdots \geq p_{\sigma}\right) \in \mathcal{P}_{n}^{+}, p_{*}^{\prime}=\left(p_{1}^{\prime} \geq p_{2}^{\prime} \geq \cdots \geq p_{\sigma}^{\prime}\right) \in \mathcal{P}_{n}^{+}$satisfy $2 p_{i}+\psi(i)=2 p_{i}^{\prime}+\psi^{\prime}(i)$ for $i \in[1, \sigma]$ (here $\psi^{\prime}$ is defined in terms of $p_{*}^{\prime}$ in the same way as $\psi$ is defined in terms of $\left.p_{*}\right)$. Since $\psi(1)=\psi^{\prime}(1)=1$ we see that $p_{1}=p_{1}^{\prime}$. Assume now that $i \geq 2$ and that $p_{j}=p_{j}^{\prime}$ for $j \in[1, i-1]$. From our assumption we have $\psi(i)=\psi^{\prime}(i) \bmod 2$. If $i$ is odd, then $\psi(i), \psi^{\prime}(i)$ belong to $\{0,1\}$; hence $\psi(i)=\psi^{\prime}(i)$ and $p_{i}=p_{i}^{\prime}$. If $i$ is even, then $\psi(i), \psi^{\prime}(i)$ belong to $\{0,-1\}$; hence $\psi(i)=\psi^{\prime}(i)$ and $p_{i}=p_{i}^{\prime}$. Thus $p_{*}=p_{*}^{\prime}$. Similarly we see that if 
$p_{*}=\left(p_{1} \geq p_{2} \geq \cdots \geq p_{\sigma}\right) \in \mathcal{P}_{n}-\mathcal{P}_{n}^{+}, p_{*}^{\prime}=\left(p_{1}^{\prime} \geq p_{2}^{\prime} \geq \cdots \geq p_{\sigma}^{\prime}\right) \in \mathcal{P}_{n}-\mathcal{P}_{n}^{+}$satisfy $2 p_{i}+\psi(i)=2 p_{i}^{\prime}+\psi^{\prime}(i)$ for $i \in[1, \sigma]$, then $p_{*}=p_{*}^{\prime}$. This proves the injectivity statement in Proposition 0.6(ii). Now let $\gamma_{0}$ be a distinguished unipotent class in $G$. Then, if $u_{0} \in \gamma_{0}$, the Jordan blocks of $u_{0}-1$ have sizes $2 x_{1}+1>2 x_{2}+1>$ $\cdots>2 x_{f}+1$, where $x_{1}>x_{2}>\cdots>x_{f}$ are integers $\geq 0$ and $f$ is odd. Let $p_{*}=\left(x_{1} \geq x_{2}+1 \geq x_{3} \geq x_{4}+1 \geq \cdots \geq x_{f-2} \geq x_{f-1}+1 \geq x_{f}\right)$ if $x_{f}>0$ and $p_{*}=\left(x_{1} \geq x_{2}+1 \geq x_{3} \geq x_{4}+1 \geq \cdots \geq x_{f-2} \geq x_{f-1}+1\right)$ if $x_{f}=0$. Then $\gamma_{p_{*}}=\gamma_{0}$. This completes the proof of Proposition 0.6 in our case.

3.9. We prove Proposition 0.6 in the case where $G$ is as in $\S 1.3$ with $\kappa=0, Q \neq$ $0, p \neq 2, \mathbf{n} \geq 8$. Let $p_{*}=\left(p_{1} \geq p_{2} \geq \cdots \geq p_{\sigma}\right) \in \mathcal{P}_{n}^{+}$. Let $w_{p_{*}} \in W^{\prime}$ be as in $\S 1.6$. Let $w=w_{p_{*}}^{-1}$. By the argument in $\S 2.12$ we can find $U_{*} \in \mathcal{F}$ and a unipotent element $u \in G$ such that $a_{U_{*}, u U_{*}}=w$ and such that, setting $N_{0}=u^{-1}-1$, the Jordan blocks of $N_{0}$ have sizes $2 p_{1}+\psi(1), 2 p_{2}+\psi(2), \ldots, 2 p_{\sigma}+\psi(s)(\psi$ as in $\S 1.6)$. We can also assume that $U_{*} \in \mathcal{F}^{\prime}$. Note that for any $k \geq 0$ we have $\operatorname{dim} N_{0}^{k}=\Lambda_{k}^{\prime \prime}$ (notation of $\S 3.6$ ). Let $\gamma_{p_{*}}$ be the $G$-conjugacy class of $u$. Let $B=B_{U_{*}}$. We have $\left(B, u B u^{-1}\right) \in \mathcal{O}_{w}$. Since $w \in\left(C_{p_{*}}^{\prime}\right)_{\text {min }}$, we have $C_{p_{*}}^{\prime} \dashv \gamma_{p_{*}}$. Now let $\gamma^{\prime} \in \underline{\underline{G}}$ be such that $C_{p_{*}}^{\prime} \dashv \gamma^{\prime}$. Then there exists $g \in \gamma^{\prime}$ such that $g B g^{-1}=u B u^{-1}$. We set $U_{*}^{\prime}=u U_{*} u^{-1}$. We have $a_{U_{*}, U_{*}^{\prime}}=w$ and $g U_{*}=U_{*}^{\prime}\left(\right.$ since $\left.g B g^{-1}=u B u^{-1}\right)$. By 3.6(c),(e) for any $k \geq 0$ we have $\operatorname{dim} N^{k} V \geq \Lambda_{k}^{\prime \prime}$; hence $\operatorname{dim} N^{k} V \geq \operatorname{dim} N_{0}^{k} V$. It follows that the conjugacy class of $u^{-1}$ in $G L(V)$ is contained in the closure of the conjugacy class of $g$ in $G L(V)$. Since $p \neq 2$ it follows that the conjugacy class of $u$ in $I s(V)$ is contained in the closure of the conjugacy class of $g$ in $I s(V)$. Hence $\gamma_{p_{*}}$ is contained in the closure of $\gamma^{\prime} \cup\left(h \gamma^{\prime} h^{-1}\right)$, where $h \in I s(V)-G$. Then either $\gamma_{p_{*}}$ is contained in the closure of $\gamma^{\prime}$ or $\gamma_{p_{*}}$ is contained in the closure of $h \gamma^{\prime} h^{-1}$. In the last case we see that $h^{-1} \gamma_{p_{*}} h$ is contained in the closure of $\gamma^{\prime}$; but since $2 p_{1}+\psi(1)$ is odd we have $h^{-1} \gamma_{p_{*}} h=\gamma_{p_{*}}$; hence we have again that $\gamma_{p_{*}}$ is contained in the closure of $\gamma^{\prime}$. We see that property $\Pi_{C_{p_{*}}^{\prime}}$ holds with $\gamma_{P_{p_{*}}^{\prime}}=\gamma_{p_{*}}$. This completes the proof of Proposition 0.6(i) in our case. The proof of the injectivity in 0.6(ii) is entirely similar to the proof in $\S 3.8$. Now let $\gamma_{0}$ be a distinguished unipotent class in $G$. Then, if $u_{0} \in \gamma_{0}$, the Jordan blocks of $u_{0}-1$ have sizes $2 x_{1}+1>2 x_{2}+1>\cdots>2 x_{f}+1$, where $x_{1}>x_{2}>\cdots>x_{f}$ are integers $\geq 0$ and $f$ is even. Let $p_{*}=\left(x_{1} \geq x_{2}+1 \geq x_{3} \geq x_{4}+1 \geq \cdots \geq x_{f-1} \geq x_{f}+1\right)$. Then $\gamma_{p_{*}}=\gamma_{0}$. This completes the proof of Proposition 0.6 in our case.

\section{Basic Unipotent ClASSES}

4.1. In this section there is no restriction on $p$. Let $G^{\prime}$ be a connected reductive group over $\mathbf{C}$ of the same type as $G$ (with the same root datum as $G$ ). Then $\underline{\mathbf{W}}$ for $G$ and $G^{\prime}$ may be identified. Let $\underline{G}^{\prime}$ be the set of unipotent classes of $G^{\prime}$. There is a well-defined map $\pi: \underline{G}^{\prime} \rightarrow \underline{G}$ given by $\pi\left(\gamma^{\prime}\right)=\gamma$, where $\gamma^{\prime}, \gamma$ correspond to the same irreducible $\mathbf{W}$-module under the Springer correspondence. This map is injective and dimension preserving. One can show that it coincides with the map described in [Sp1, III. 5.2]. Let $\tilde{\Phi}^{\prime}: \underline{\mathbf{W}}_{e l} \rightarrow \underline{G}^{\prime}$ be the (injective) map $C \mapsto \gamma_{C}$ (as in Proposition 0.6, for $G^{\prime}$ instead of $G$ ). Let $\tilde{\Phi}=\pi \tilde{\Phi}^{\prime}: \underline{\mathbf{W}}_{e l} \rightarrow \underline{\underline{G}}$, be an injective map. (When $p$ is not a bad prime for $G$, then $\tilde{\Phi}$ is given by $C \mapsto \gamma_{C}$ (as in Proposition 0.6). This follows from the explicit computation of the map $C \mapsto \gamma_{C}$; see below.) A unipotent class of $G$ is said to be basic if it is in the image of $\tilde{\Phi}$. Let 
$\underline{\underline{G}}_{b}$ be the set of basic unipotent classes of $G$. Note that $\pi$ restricts to a bijection $\underline{\underline{G}}_{b}^{\prime} \stackrel{\sim}{\longrightarrow} \underline{\underline{G}}_{b}$. Let $\Phi: \underline{\mathbf{W}}_{e l} \stackrel{\sim}{\longrightarrow} \underline{\underline{G}}_{b}$ be the restriction of $\tilde{\Phi}$.

4.2. We shall need the following definition. Let $V,(), Q, n,, \kappa, G$ be as in $\S 1.3$ and let $p_{*}=\left(p_{1} \geq p_{2} \geq \cdots \geq p_{\sigma}\right)$ be in $\mathcal{P}_{n}$ (if $\left.(1-\kappa) Q=0\right)$ and in $\mathcal{P}_{n}^{+}$(if $\left.(1-\kappa) Q \neq 0\right)$. Let $\psi$ be as in $\S 1.6$. Let $\gamma_{p_{*}}$ be the unipotent class in $G$ such that for some/any $g \in \gamma_{p_{*}}$ the Jordan blocks of $g-1$ have sizes

$2 p_{1} \geq 2 p_{2} \geq \cdots \geq 2 p_{\sigma}$ if $\kappa=0, Q=0$ or if $\kappa=0, Q \neq 0, p=2$,

$2 p_{1} \geq 2 p_{2} \geq \cdots \geq 2 p_{\sigma} \geq 1$ if $\kappa=1, Q \neq 0, p=2$,

$2 p_{1}+\psi(1) \geq 2 p_{2}+\psi(2) \geq \cdots \geq 2 p_{\sigma}+\psi(\sigma)$ if $\kappa=1, Q \neq 0, p \neq 2, \sigma=$ odd or if $\kappa=0, Q \neq 0, p \neq 2$,

$2 p_{1}+\psi(1) \geq 2 p_{2}+\psi(2) \geq \cdots \geq 2 p_{\sigma}+\psi(\sigma) \geq 1$ if $\kappa=1, Q \neq 0, p \neq 2, \sigma=$ even, and such that (if $p=2$ ):

(a) for any $i \in[1, \sigma]$ we have $\left((g-1)^{2 p_{i}-1} x, x\right) \neq 0$ for some $x \in \operatorname{ker}(g-1)^{2 p_{i}}$. Note that in each case the unipotent conjugacy class $\gamma_{p_{*}}$ is well defined.

4.3. We now describe the bijection $\Phi: \underline{\mathbf{W}}_{e l} \stackrel{\sim}{\longrightarrow} \underline{\underline{G}}_{b}$ for $G$ almost simple of various types.

If $G$ is of type $A_{n}$, then $\Phi\left(C_{c o x}\right)$ is the regular unipotent class.

If $V,(), Q, n,, \kappa, G$ are as in $\S 1.3$ and $p_{*}=\left(p_{1} \geq p_{2} \geq \cdots \geq p_{\sigma}\right)$ is in $\mathcal{P}_{n}$ (if $(1-\kappa) Q=0)$ and in $\mathcal{P}_{n}^{+}($if $(1-\kappa) Q \neq 0)$, then $\Phi\left(C_{p_{*}}\right)=\gamma_{p_{*}}($ if $(1-\kappa) Q=0)$ and $\Phi\left(C_{p_{*}}^{\prime}\right)=\gamma_{p_{*}}($ if $(1-\kappa) Q \neq 0)$.

When $G$ is of exceptional type we use the notation of [Mi], [Sp2 for the unipotent classes in $G$; an element $C \in \underline{\mathbf{W}}_{e l}$ is specified by indicating the characteristic polynomial of an element of $C$ acting on the reflection representation of $\mathbf{W}$, a product of cyclotomic polynomials $\Phi_{d}$ (an exception is type $F_{4}$ when there are two choices for $C$ with characteristic polynomial $\Phi_{2}^{2} \Phi_{6}$, in which case we use the notation $\left(\Phi_{2}^{2} \Phi_{6}\right)^{\prime},\left(\Phi_{2}^{2} \Phi_{6}\right)^{\prime \prime}$ for what in [GP, p.407] is denoted by $\left.D_{4}, C_{3}+A_{1}\right)$. The notation $d ; C ; \gamma$ below means that $C \in \underline{\mathbf{W}}_{e l}, \gamma \in \underline{\underline{G}}_{b}, \Phi(C)=\gamma, d=d_{C}$ (see $\S 0.2$ ). A symbol dist is added when $\gamma$ is distinguished for any $p$; a symbol dist $t_{p}$ is added when $\gamma$ is distinguished only for the specified $p$. The values of $d_{C}$ are taken from $\mathrm{GP}$.

Type $G_{2}$.

$2 ; \Phi_{6} ; G_{2}$ dist

$4 ; \Phi_{3} ; G_{2}\left(a_{1}\right) \quad$ dist

$6 ; \Phi_{2}^{2} ; \tilde{A}_{1} \quad$ dist $_{3}$

Type $F_{4}$.

$4 ; \Phi_{12} ; F_{4}$ dist

$6 ; \Phi_{8} ; F_{4}\left(a_{1}\right)$ dist

$8 ; \Phi_{6}^{2} ; F_{4}\left(a_{2}\right)$ dist

$10 ;\left(\Phi_{2}^{2} \Phi_{6}\right)^{\prime} ; B_{3}$

$10 ;\left(\Phi_{2}^{2} \Phi_{6}\right)^{\prime \prime} ; C_{3}$

$12 ; \Phi_{4}^{2} ; F_{4}\left(a_{3}\right) \quad$ dist

$14 ; \Phi_{2}^{2} \Phi_{4} ; C_{3}\left(a_{1}\right) \quad$ dist $_{2}$

$16 ; \Phi_{3}^{2} ; \tilde{A}_{2}+A_{1} \quad$ dist $_{2}$

$24 ; \Phi_{2}^{4} ; A_{1}+\tilde{A}_{1}$ 
Type $E_{6}$.

$6 ; \Phi_{3} \Phi_{12} ; E_{6}$ dist

$8 ; \Phi_{9} ; E_{6}\left(a_{1}\right) \quad$ dist

$12 ; \Phi_{3} \Phi_{6}^{2} ; A_{5}+A_{1} \quad$ dist

$14 ; \Phi_{2}^{2} \Phi_{3} \Phi_{6} ; A_{5}$

$24 ; \Phi_{3}^{3} ; 2 A_{2}+A_{1}$

Type $E_{7}$.

$7 ; \Phi_{2} \Phi_{18} ; E_{7} \quad$ dist

$9 ; \Phi_{2} \Phi_{14} ; E_{7}\left(a_{1}\right) \quad$ dist

$11 ; \Phi_{2} \Phi_{6} \Phi_{12} ; E_{7}\left(a_{2}\right) \quad$ dist

$13 ; \Phi_{2} \Phi_{6} \Phi_{10} ; D_{6}+A_{1} \quad$ dist

$15 ; \Phi_{2}^{3} \Phi_{10} ; D_{6}$

$17 ; \Phi_{2} \Phi_{4} \Phi_{8} ; D_{6}\left(a_{1}\right)+A_{1} \quad$ dist

$21 ; \Phi_{2} \Phi_{6}^{3} ; D_{6}\left(a_{2}\right)+A_{1}$ dist

$23 ; \Phi_{2}^{3} \Phi_{6}^{2} ; D_{6}\left(a_{2}\right)$

$25 ; \Phi_{2} \Phi_{3}^{2} \Phi_{6} ;\left(A_{5}+A_{1}\right)^{\prime \prime}$

$31 ; \Phi_{2}^{5} \Phi_{6} ; D_{4}+A_{1}$

$33 ; \Phi_{2}^{3} \Phi_{4}^{2} ; A_{3}+A_{2}+A_{1}$

$63 ; \Phi_{2}^{7} ; 4 A_{1}$

Type $E_{8}$.

$8 ; \Phi_{30} ; E_{8}$ dist

$10 ; \Phi_{24} ; E_{8}\left(a_{1}\right) \quad$ dist

$12 ; \Phi_{20} ; E_{8}\left(a_{2}\right) \quad$ dist

$14 ; \Phi_{6} \Phi_{18} ; E_{7}+A_{1} \quad$ dist

$16 ; \Phi_{15} ; D_{8} \quad$ dist

$16 ; \Phi_{2}^{2} \Phi_{18} ; E_{7}$

$18 ; \Phi_{2}^{2} \Phi_{14} ; E_{7}\left(a_{1}\right)+A_{1} \quad$ dist

$20 ; \Phi_{12}^{2} ; D_{8}\left(a_{1}\right) \quad$ dist

$22 ; \Phi_{4}^{2} \Phi_{12} ; D_{7}$

$22 ; \Phi_{6}^{2} \Phi_{12} ; E_{7}\left(a_{2}\right)+A_{1} \quad$ dist

$24 ; \Phi_{10}^{2} ; A_{8}$ dist

$24 ; \Phi_{2}^{2} \Phi_{6} \Phi_{12} ; E_{7}\left(a_{2}\right)$

$26 ; \Phi_{3}^{2} \Phi_{12} ; E_{6}+A_{1}$

$26 ; \Phi_{2}^{2} \Phi_{6} \Phi_{10} ; D_{7}\left(a_{1}\right) \quad$ dist $_{2}$

$28 ; \Phi_{3} \Phi_{9} ; D_{8}\left(a_{3}\right) \quad$ dist

$30 ; \Phi_{8}^{2} ; A_{7} \quad$ dist $_{3}$

$32 ; \Phi_{2}^{4} \Phi_{10} ; D_{6}$

$34 ; \Phi_{2}^{2} \Phi_{4} \Phi_{8} ; D_{5}+A_{2} \quad$ dist $_{2}$

$40 ; \Phi_{6}^{4} ; 2 A_{4}$ dist

$42 ; \Phi_{2}^{2} \Phi_{6}^{3} ; A_{5} A_{2}$

$44 ; \Phi_{2}^{4} \Phi_{6}^{2} ; D_{6}\left(a_{2}\right)$

44; $\Phi_{3}^{2} \Phi_{6}^{2} ; A_{5}+2 A_{1}$

$46 ; \Phi_{2}^{2} \Phi_{3}^{2} \Phi_{6} ;\left(A_{5}+A_{1}\right)^{\prime}$

$46 ; \Phi_{2}^{2} \Phi_{4}^{2} \Phi_{6} ; D_{5}\left(a_{1}\right)+A_{2}$

$48 ; \Phi_{5}^{2} ; A_{4}+A_{3}$

$60 ; \Phi_{4}^{4} ; 2 A_{3}$ 


$$
\begin{aligned}
& 64 ; \Phi_{2}^{6} \Phi_{6} ; D_{4}+A_{1} \\
& 66 ; \Phi_{2}^{4} \Phi_{4}^{2} ; A_{3}+A_{2}+A_{1} \\
& 80 ; \Phi_{3}^{4} ; 2 A_{2}+2 A_{1} \\
& 120 ; \Phi_{2}^{8} ; 4 A_{1}
\end{aligned}
$$

4.4. We have the following result.

(a) If $\gamma$ is a distinguished unipotent class of $G$, then $\gamma$ is a basic unipotent class of $G$.

This follows from the known classification of distinguished unipotent classes Mi], Sp1 and the results in $\S 4.3$. For example if $V,(), Q, n,, \kappa, G$ are as in $\S 1.3$ with $p=2$ and $p_{*}=\left(p_{1} \geq p_{2} \geq \cdots \geq p_{\sigma}\right)$ is in $\mathcal{P}_{n}($ if $(1-\kappa) Q=0)$ and in $\mathcal{P}_{n}^{+}$ (if $(1-\kappa) Q \neq 0$ ), then $\gamma_{p_{*}}$ is distinguished if and only if for any $j \geq 1$ we have $\sharp\left(i \in[1, \sigma] ; 2 p_{i}=j\right) \leq 2$ (and all distinguished classes are of this form).

Next we note the following result:

(b) Let $C \in \underline{\mathbf{W}}_{\text {el }}$ and let $g \in \Phi(C)$. If $G$ is semisimple, then $\operatorname{dim}(Z(g))$ is equal to $d_{C}$ (the minimum value of the length function on $C$ ).

When $G$ is almost simple of type $A_{n}$, this is obvious. When $G$ is almost simple of exceptional type this follows from the results in $\$ 4.3$ and from Mi. Now assume that $V,(), Q, n,, \kappa, G$ are as in $\S 1.3$ and $p_{*}=\left(p_{1} \geq p_{2} \geq \cdots \geq p_{\sigma}\right.$ ) is in $\mathcal{P}_{n}$ (if $(1-\kappa) Q=0)$ and in $\mathcal{P}_{n}^{+}($if $(1-\kappa) Q \neq 0)$. Let $d^{\prime}=\operatorname{dim} Z(g)$. Using $\S \S 2.2,2.3$ we see that it is enough to show that

(c) $d^{\prime}=2 \sum_{v=1}^{\sigma-1} v p_{v+1}+n($ if $(1-\kappa) Q=0)$ and $d^{\prime}=2 \sum_{v=1}^{\sigma-1} v p_{v+1}+n-\sigma$ (if $(1-\kappa) Q \neq 0)$.

Since $\operatorname{dim}(\pi(\gamma))=\operatorname{dim} \gamma$ for any $\gamma \in \underline{G^{\prime}}$ (notation of $\S 4.1$ ) we see that it is enough to prove (c) in the case where $p=2$. Using the exceptional isogeny from type $B_{n}$ to type $C_{n}$ we see that (c) in type $B_{n}$ follows from (c) in type $C_{n}$. Using Sp1, II, 6.4, 6.5] we see that (c) in type $D_{n}$ follows from (c) in type $C_{n}$. Thus we may assume that $\kappa=0, Q=0, p=2$. For $j \geq 1$ let $f_{j}=\sharp\left(i \in[1, \sigma] ; 2 p_{i} \geq j\right)$. By Sp1, II, $6.3,6.5]$ we have

$$
d^{\prime}=\sum_{h \geq 1}\left(f_{2 h}^{2}-f_{2 h}\right)+n
$$

since $f_{2 h-1}=f_{2 h}$. It remains to prove the identity $X=2 Y$, where

$$
X=\sum_{h \geq 1}\left(f_{2 h}^{2}-f_{2 h}\right), \quad Y=p_{2}+2 p_{3}+\cdots+(\sigma-1) p_{\sigma} .
$$

We can find integers $a_{1}, a_{2}, \ldots, a_{t}, b_{1}, b_{2}, \ldots, b_{t}$ (all $\left.\geq 1\right)$ such that $p_{i}=a_{1}+a_{2}+$ $\cdots+a_{t}$ for $i \in\left[1, b_{1}\right], p_{i}=a_{1}+a_{2}+\cdots+a_{t-1}$ for $i \in\left[b_{1}+1, b_{1}+b_{2}\right], \ldots, p_{i}=a_{1}$ for $i \in\left[b_{1}+b_{2}+\cdots+b_{t-1}+1, b_{1}+b_{2}+\cdots+b_{t}\right]$. We have

$$
\begin{aligned}
X=a_{1}\left(\left(b_{1}+\right.\right. & \left.\left.b_{2}+\cdots+b_{t}\right)^{2}-\left(b_{1}+b_{2}+\cdots+b_{t}\right)\right) \\
+ & a_{2}\left(\left(b_{1}+b_{2}+\cdots+b_{t-1}\right)^{2}-\left(b_{1}+b_{2}+\cdots+b_{t-1}\right)\right)+\cdots+a_{t}\left(b_{1}^{2}-b_{1}\right), \\
Y= & \left(a_{1}+a_{2}+\cdots+a_{t}\right)\left(b_{1}^{2}-b_{1}\right) / 2 \\
& +\left(a_{1}+a_{2}+\cdots+a_{t-1}\right)\left(\left(b_{2}^{2}-b_{2}\right) / 2-\left(b_{1}^{2}-b_{1}\right) / 2\right)+\ldots \\
& +a_{1}\left(\left(b_{t}^{2}-b_{t}\right) / 2-\left(b_{t-1}^{2}-b_{t-1}\right) / 2\right) .
\end{aligned}
$$

The equality $X=2 Y$ follows. This completes the proof of (b). 
4.5. We now define a map $\Phi: \underline{\mathbf{W}} \rightarrow \underline{\underline{G}}$ extending the map $\Phi: \underline{\mathbf{W}}_{e l} \rightarrow \underline{G}_{b}$ in $\S 4.1$. Let $C \in \underline{\mathbf{W}}$. We can find $J \subset S$ and an elliptic conjugacy class $D$ of the Weyl group $\mathbf{W}_{J}$ such that $D=C \cap \mathbf{W}_{J}$. Let $P$ be a parabolic subgroup of $G$ of type $J$. Let $L$ be a Levi subgroup of $P$. Let $\gamma_{D}=\Phi_{L}(D)$, a unipotent class of $L$ (here $\Phi_{L}$ is the map $\Phi$ of $\S 4.1$ with $G, \mathbf{W}$ replaced by $\left.L, \mathbf{W}_{J}\right)$. Let $\gamma$ be the unipotent class of $G$ containing $\gamma_{D}$. We set $\Phi(C)=\gamma$. We show that $\gamma$ is independent of the choices made. Assume that we also have $D^{\prime}=C \cap \mathbf{W}_{J^{\prime}}$, where $J^{\prime} \subset S$ and $D^{\prime}$ is an elliptic conjugacy class of the Weyl group $\mathbf{W}_{J^{\prime}}$. Let $P^{\prime}$ be a parabolic subgroup of $G$ of type $J^{\prime}$. Let $L^{\prime}$ be a Levi subgroup of $P$. Let $\gamma_{D^{\prime}}=\Phi_{L^{\prime}}\left(D^{\prime}\right)$, a unipotent class of $L^{\prime}$. Let $\gamma^{\prime}$ be the unipotent class of $G$ containing $\gamma_{D^{\prime}}$. We must show that $\gamma=\gamma^{\prime}$. By [GP, 3.2.12] there exists $x \in \mathbf{W}$ such that $x J x^{-1}=J^{\prime}$ and $x D x^{-1}=D^{\prime}$. We can find an element $\dot{x} \in G$ such that $\dot{x} L \dot{x}^{-1}=L^{\prime}$ and such that conjugation by $\dot{x}$ induces the isomorphism $\mathbf{W}_{J} \rightarrow \mathbf{W}_{J^{\prime}}$ given by $w \mapsto x w x^{-1}$. By functoriality we must have $\dot{x} \Phi_{L}(D) \dot{x}^{-1}=\Phi_{L^{\prime}}\left(D^{\prime}\right)$. It follows that $\dot{x} \gamma \dot{x}^{-1}=\gamma^{\prime}$; hence $\gamma=\gamma^{\prime}$. We see that $C \mapsto \Phi(C)$ is a well-defined map $\underline{\mathbf{W}} \rightarrow \underline{G}$; it clearly extends the map $\Phi: \underline{\mathbf{W}}_{e l} \rightarrow \underline{\underline{G}}_{b}$ in $\S 4.1$. Also, if $p$ is not a bad prime for $G$, then the map $\underline{\mathbf{W}} \rightarrow \underline{\underline{G}}$ just defined coincides with the map $\underline{\mathbf{W}} \rightarrow \underline{G}$ given by Theorem 0.4. (This follows from §1.1.)

Note that $\Phi: \underline{\mathbf{W}} \rightarrow \underline{\underline{G}}$ can be described explicitly for any $G$ using the description of the bijections $\underline{\mathbf{W}}_{e l} \stackrel{\stackrel{\vec{\sigma}}{\longrightarrow}}{\underline{G}_{b}}$ given in $\S 4.3$ (with $G$ replaced by a Levi subgroup of a parabolic subgroup of $G$ ).

We show that

(a) $\Phi: \underline{\mathbf{W}} \rightarrow \underline{\underline{G}}$ is surjective.

Let $\gamma \in \underline{G}$. We can find a parabolic subgroup $P$ of $G$ with Levi subgroup $L$ and a distinguished unipotent class $\gamma_{1}$ of $L$ such that $\gamma_{1} \subset \gamma$. Let $J$ be the subset $S$ such that $P$ is of type $J$. By 4.4(a), $\gamma_{1}$ is a basic unipotent class of $L$. Hence we can find an elliptic conjugacy class $D$ of $\mathbf{W}_{J}$ such that $\Phi_{L}(D)=\gamma_{1}\left(\Phi_{L}\right.$ is the map $\Phi$ of $\S 4.1$ with $G, \mathbf{W}$ replaced by $\left.L, \mathbf{W}_{J}\right)$. Let $C$ be the conjugacy class in $\mathbf{W}$ that contains $D$. By the arguments above we have $\Phi(C)=\gamma$. This proves (a).

4.6. In this subsection we show, assuming that all simple factors of $G$ are of type $A_{n}, B_{n}, C_{n}$ or $D_{n}$, that a part of Theorem 0.4(i) holds for the map $\Phi: \underline{\mathbf{W}} \rightarrow \underline{\underline{G}}$ even in bad characteristic.

(a) Let $C \in \underline{\mathbf{W}}$ and let $\gamma=\Phi(C) \in \underline{G}$. Then $C \dashv \gamma$.

As in $\S 1.1$ we can assume that $C \in \underline{\mathbf{W}} \overline{\bar{e}_{l}}$. If $p \neq 2$ the result follows from Proposition 0.6. We now assume that $p=2$. We can also assume that $G$ is almost simple of type $\neq A_{n}$. We can now assume that $V,(), Q, n,, \kappa, G$ are as in $\S 1.3$ with $p=2$ and that $C=C_{p_{*}}$ (if $\left.(1-\kappa) Q=0\right)$ and $C=C_{p_{*}}^{\prime}$ (if $\left.(1-\kappa) Q \neq 0\right)$, where $p_{*}=\left(p_{1} \geq p_{2} \geq \cdots \geq p_{\sigma}\right)$ is in $\mathcal{P}_{n}$ (if $\left.(1-\kappa) Q=0\right)$ and in $\mathcal{P}_{n}^{+}($if $(1-\kappa) Q \neq 0)$. Using the exceptional bijection from type $B_{n}$ to type $C_{n}$ we see that the result for type $B_{n}$ follows from the result in type $C_{n}$. Thus we can assume in addition that $\kappa=0$. As in the proof in $\S \S 3.7,3.9$ we see that there exists $\gamma \in \underline{\underline{G}}$ such that if $g \in \gamma$, then $g-1$ has Jordan blocks of sizes $2 p_{1} \geq 2 p_{2} \geq \cdots \geq 2 p_{\sigma}$ and $C \dashv \gamma$. It remains to show that $g$ satisfies the conditions 4.2(a). It is enough to show that there exists a direct sum decomposition $V=V^{1} \oplus V^{2} \oplus \ldots \oplus V^{m}$ such that $\left(V^{i}, V^{j}\right)=0$ for $i \neq j, V^{i} \oplus\left(V^{i}\right)^{\perp}=V$ for each $i$ and such that for each $i, V^{i}$ is $g$-stable and $N:=g-1: V^{i} \rightarrow V^{i}$ has a single Jordan block. To do this we use 3.5(c) (applied 
to $V_{*} \in \mathcal{F}$ or $V_{*} \in \mathcal{F}^{\prime}$ such that $\left.a_{V_{*}, g V_{*}}=w_{p_{*}}\right)$ and we take for $V^{i}$ the subspaces $X_{r}$ in $\S 3.4$ for $r \in[1, \sigma]$. This completes the proof of (a).

We expect that (a) holds without any assumption on $G$.

4.7. Let $C \in \underline{\mathbf{W}}_{e l}$ and let $w \in C_{\min }$. Define $u_{w} \in G$ in terms of any excellent decomposition of $w$ as in $\S 2.4$. We conjecture that $u_{w} \in \Phi(C)$. This is supported by the computations in Section 2 .

4.8. Assume that $\mathbf{k}=\mathbf{C}$ and consider the bijection $\underline{\underline{G}}_{b} \stackrel{\sim}{\longrightarrow} \underline{\mathbf{W}}_{e l}$ inverse to $\Phi$. We expect that this coincides with the restriction of the map $\underline{G} \rightarrow \underline{\mathbf{W}}$ defined in $\mathrm{KL}$. (This holds in every case in which the last map has been computed; see [Sp3, Sp4]. In particular it holds when $G$ is as in $§ 1.3$.)

\section{C-SMAll Classes}

5.1. In this section we fix $C \in \underline{\mathbf{W}}_{e l}$.

Let $\mathcal{R}$ be the reflection representation of $\mathbf{W}$. Then $\operatorname{det}(1-w, \mathcal{R}) \in \mathbf{N}_{>0}$. If $p>1$ we denote by $\operatorname{det}(1-w, \mathcal{R})^{*}$ the part prime to $p$ of $\operatorname{det}(1-w, \mathcal{R})$; if $p=0$ we set $\operatorname{det}(1-w, \mathcal{R})^{*}=\operatorname{det}(1-w, \mathcal{R})$. We have the following result.

Theorem 5.2. The isotropy groups of the $G_{a d}$-action 0.2 on $\mathfrak{B}_{w}$ are finite abelian of order dividing $\operatorname{det}(1-w, \mathcal{R})^{*}$.

For the proof we shall need the following result, which will be proved in $\S 5.3$.

(a) If $w^{\prime}, w^{\prime \prime} \in C_{\min }$, then there exists an isomorphism $\mathfrak{B}_{w^{\prime}} \stackrel{\sim}{\longrightarrow} \mathfrak{B}_{w^{\prime \prime}}$ commuting with the $G_{a d}$-actions and commuting with the first projections $\mathfrak{B}_{w^{\prime}} \rightarrow G, \mathfrak{B}_{w^{\prime \prime}} \rightarrow G$. Let $d$ be the order of $w$. Using (a) and a result of Geck and Michel [GP, 4.3.5] we see that we can assume that $w$ is a "good element" in the sense of [GP, 4.3.1]. Let $\beta^{+}$be the braid monoid attached to the Coxeter group W. Let $w_{1} \mapsto \hat{w}_{1}$ be the canonical imbedding $\mathbf{W} \rightarrow \beta^{+}$; see [GP, 4.1.1]. Let $(\hat{w})^{d}$ be the $d$-th power of $\hat{w}$ in $\beta^{+}$. Let $w_{0}$ be the longest element of $\mathbf{W}$. Since $w$ is good there exists $z \in \beta^{+}$such that $(\hat{w})^{d}=\hat{w}_{0} z$ in $\beta^{+}$. Let $s_{1} s_{2} \ldots s_{k}$ be a reduced expression of $w$. Let $s_{1}^{\prime} s_{2}^{\prime} \ldots s_{f}^{\prime}$ be a reduced expression of $w_{0}$. We can find a sequence $s_{1}^{\prime \prime}, s_{2}^{\prime \prime}, \ldots, s_{h}^{\prime \prime}$ in $S$ such that $z=\hat{s}_{1}^{\prime \prime} \hat{s}_{2}^{\prime \prime} \ldots \hat{s}_{h}^{\prime \prime}$. We have

$$
\left(\hat{s}_{1} \hat{s}_{2} \ldots \hat{s}_{k}\right)\left(\hat{s}_{1} \hat{s}_{2} \ldots \hat{s}_{k}\right) \ldots\left(\hat{s}_{1} \hat{s}_{2} \ldots \hat{s}_{k}\right)=\hat{s}_{1}^{\prime} \hat{s}_{2}^{\prime} \ldots \hat{s}_{f}^{\prime} \hat{s}_{1}^{\prime \prime} \hat{s}_{2}^{\prime \prime} \ldots \hat{s}_{h}^{\prime \prime}
$$

(The left hand side contains $k d$ factors $\hat{s}_{i}$. The right hand side contains $f+h$ factors.) We must have $k d=f+h$. Moreover, from the definition of $\beta^{+}$there exist $\mathbf{s}^{1}, \mathbf{s}^{2}, \ldots, \mathbf{s}^{m}(m \geq 2)$ such that each $\mathbf{s}^{r}$ is a sequence $\mathbf{s}_{1}^{r}, \mathbf{s}_{2}^{r}, \ldots, \mathbf{s}_{k d}^{r}$ in $S$, $\mathbf{s}^{1}$ is the sequence $s_{1}, s_{2}, \ldots, s_{k}, s_{1}, s_{2}, \ldots, s_{k}, \ldots, s_{1}, s_{2}, s_{k}$ ( $k d$ terms), $\mathbf{s}^{m}$ is the sequence $s_{1}^{\prime}, s_{2}^{\prime}, \ldots, s_{f}^{\prime}, s_{1}^{\prime \prime}, s_{2}^{\prime \prime}, s_{h}^{\prime \prime}$ and for any $r \in[1, m-1]$ the sequence $\mathbf{s}^{r+1}$ is obtained from the sequence $\mathbf{s}^{r}$ by replacing a string $\mathbf{s}_{e+1}^{r}, \mathbf{s}_{e+2}^{r}, \ldots, \mathbf{s}_{e+u}^{r}$ of the form $s, t, s, t, \ldots$ ( $u$ terms, $s \neq t$ in $S$, st of order $u$ in $\mathbf{W})$ by the string $t, s, t, s, \ldots(u$ terms). Now let $(g, B) \in \mathfrak{B}_{w}$, let $\mathfrak{Z}=\left\{c \in G: c g c^{-1}=g, c B c^{-1}=B\right\}$ and let $c \in \mathfrak{Z}$. We define a sequence $B_{0}, B_{1}, \ldots, B_{k d}$ in $\mathcal{B}$ by the following requirements: $B_{i k}=g^{i} B g^{-i}$ for $i \in[0, d],\left(B_{i k+j-1}, B_{i k+j}\right) \in \mathcal{O}_{s_{j}}$ for $i \in[0, d-1], j \in[1, k]$. This sequence is uniquely determined. Now conjugation by $c$ preserves each of $B, g B g^{-1}, g^{2} B g^{-2}, \ldots, g^{d} B g^{-d}$; hence (by uniqueness) it automatically preserves each $B_{v}, v \in[0, k d]$. We define a sequence $B_{*}^{1}, B_{*}^{2}, \ldots, B_{*}^{m}$ such that each $B_{*}^{r}$ is a 
sequence $\left(B_{0}^{r}, B_{1}^{r}, \ldots, B_{k d}^{r}\right)$ in $\mathcal{B}$ satisfying $\left(B_{j-1}^{r}, B_{j}^{r}\right) \in \mathcal{O}_{\mathbf{s}_{j}^{r}}$ for $j \in[1, k d]$, as follows: $B_{*}^{1}=\left(B_{0}, B_{1}, \ldots, B_{h d}\right)$ and for $r \in[1, m-1], B_{*}^{r+1}$ is obtained from $B_{*}^{r}$ by replacing the string $B_{e}^{r}, B_{e+1}^{r}, \ldots, B_{e+u}^{r}\left(\right.$ where $\left(\mathbf{s}_{e+1}^{r}, \mathbf{s}_{e+2}^{r}, \ldots, \mathbf{s}_{e+u}^{r}\right)=(s, t, s, t, \ldots)$ as above) by the string $B_{e}^{r+1}, B_{e+1}^{r+1}, \ldots, B_{e+u}^{r+1}$ defined by

$$
\begin{aligned}
& B_{e}^{r+1}=B_{e}^{r}, B_{e+u}^{r+1}=B_{e+u}^{r},\left(B_{e}^{r+1}, B_{e+1}^{r+1}\right) \in \mathcal{O}_{t},\left(B_{e+1}^{r+1}, B_{e+2}^{r+1}\right) \in \mathcal{O}_{s}, \\
& \left(B_{e+2}^{r+1}, B_{e+3}^{r+1}\right) \in \mathcal{O}_{t}, \ldots
\end{aligned}
$$

(Note that $B_{e}^{r+1}, B_{e+1}^{r+1}, \ldots, B_{e+u}^{r+1}$ are uniquely determined since $\left(B_{e}^{r}, B_{e+u}^{r}\right) \in$ $\mathcal{O}_{\text {stst } \ldots}=\mathcal{O}_{t s t s \ldots}$ and stst .., tsts .. are reduced expressions in W.) We note that for any $r \in[1, m]$ any Borel subgroup in the sequence $B_{*}^{r}$ is stable under conjugation by $c$. (For $r=1$ this has already been observed. The general case follows by induction on $r$ using the uniqueness in the previous sentence.) In particular any Borel subgroup in the sequence $B_{*}^{m}$ is stable under conjugation by $c$. From the definitions we see that $\left(B_{0}^{m}, B_{f}^{m}\right) \in \mathcal{O}_{w_{0}}$, that is, $B_{0}^{m}, B_{f}^{m}$ are opposed Borel subgroups. Since both are stable under conjugation by $c$ we see that $c$ belongs to $B_{0}^{m} \cap B_{f}^{m}$, a maximal torus independent of $c$. We see that $\mathfrak{Z}$ is contained in the torus $B_{0}^{m} \cap B_{f}^{m}$. Hence $\mathfrak{Z}$ is a diagonalizable group.

Now if $c \in \mathfrak{Z}$, then $c B c^{-1}=B$ and $c g B g^{-1} c^{-1}=g B g^{-1}$; hence $c \in B \cap g B g^{-1}$. Thus $\mathfrak{Z}$ is a diagonalizable subgroup of the connected solvable group $B \cap g B g^{-1}$. Hence we can find a maximal torus $T$ of $B \cap g B g^{-1}$ such that $\mathfrak{Z} \subset T$. We can find $a, a^{\prime}$ in $U_{B}$ and $y$ in the normalizer of $T$ such that $g=a y a^{\prime}$; moreover $y$ is uniquely determined. For $c \in \mathfrak{Z}$ we have $g=c g c^{-1}=c a c^{-1} c y c^{-1} c a^{\prime} c^{-1}$. Since $c \in T$ we see that $c a c^{-1}, c a^{\prime} c^{-1}$ belong to $U_{B}$ and $c y c^{-1}$ belongs to the normalizer of $T$. By the uniqueness statement above we see that $c y c^{-1}=y$. Thus $\mathfrak{Z}$ is contained in $T^{y}$ (the fixed point set of $\operatorname{Ad}(y): T \rightarrow T)$. Let $\overline{\mathfrak{Z}}, \bar{T}, \bar{y}$ be the image of $\mathfrak{Z}, T, y$ in $G_{a d}$. Then $\overline{\mathfrak{Z}}$ is contained in $\bar{T}^{\bar{y}}$ (the fixed point set of $\operatorname{Ad}(\bar{y}): \bar{T} \rightarrow \bar{T}$ ). Since the conjugacy class of $w$ is elliptic and $\left(B, g B g^{-1}\right) \in \mathcal{O}_{w}$ (that is, $\left.\left(B, y B y^{-1}\right) \in \mathcal{O}_{w}\right)$ we see that $\bar{T}^{\bar{y}}$ is a finite abelian group of order $\operatorname{det}(1-w, \mathcal{R})^{*}$. Hence $\overline{\mathfrak{Z}}$ is a finite abelian group of order dividing $\operatorname{det}(1-w, \mathcal{R})^{*}$. This completes the proof.

5.3. We prove 5.2(a). By [GP 3.2.7(P2)], there exists a sequence $w^{\prime}=w_{1}, w_{2}, \ldots$, $w_{n}=w^{\prime \prime}$ in $W$ such that for $i \in[1, n-1]$ we have $w_{i}=b_{i} c_{i}, w_{i+1}=c_{i} b_{i}$, where $b_{i}, c_{i}$ in $W$ satisfy $\underline{l}\left(b_{i}\right)+\underline{l}\left(c_{i}\right)=\underline{l}\left(b_{i} c_{i}\right)=\underline{l}\left(c_{i} b_{i}\right)$. Hence we may assume that $w^{\prime}=b c, w^{\prime \prime}=c b$ where $b, c$ in $W$ satisfy $\underline{l}(b)+\underline{l}(c)=\underline{l}(b c)=\underline{l}(c b)$. If $(g, B) \in \mathfrak{B}_{b c}$, then there is a unique $B^{\prime} \in \mathcal{B}$ such that $\left(B, B^{\prime}\right) \in \mathcal{O}_{b},\left(B^{\prime}, g B g^{-1}\right) \in \mathcal{O}_{c}$. We have $\left(g B g^{-1}, g B^{\prime} g^{-1}\right) \in \mathcal{O}_{b}$; hence $\left(B^{\prime}, g B^{\prime} g^{-1}\right) \in \mathcal{O}_{c b}$ so that $\left(g, B^{\prime}\right) \in \mathfrak{B}_{c b}$. Thus we have defined a morphism $\alpha: \mathfrak{B}_{b c} \rightarrow \mathfrak{B}_{c b},(g, B) \mapsto\left(g, B^{\prime}\right)$. Similarly if $\left(g, B^{\prime}\right) \in \mathfrak{B}_{c b}$ there exist a unique $B^{\prime \prime} \in \mathcal{B}$ such that $\left(B^{\prime}, B^{\prime \prime}\right) \in \mathcal{O}_{c},\left(B^{\prime \prime}, g B^{\prime} g^{-1}\right) \in$ $\mathcal{O}_{b}$; we have $\left(g, B^{\prime \prime}\right) \in \mathfrak{B}_{b c}$. Thus we have defined a morphism $\alpha^{\prime}: \mathfrak{B}_{c b} \rightarrow \mathfrak{B}_{b c}$, $\left(g, B^{\prime}\right) \mapsto\left(g, B^{\prime \prime}\right)$. From the definition it is clear that $\alpha^{\prime} \alpha(g, B)=\left(g, g B g^{-1}\right)$ for all $(g, B) \in \mathfrak{B}_{b c}$ and $\alpha \alpha^{\prime}\left(g, B^{\prime}\right)=\left(g, g B^{\prime} g^{-1}\right)$ for all $\left(g, B^{\prime}\right) \in \mathfrak{B}_{c b}$. It follows that $\alpha, \alpha^{\prime}$ are isomorphisms. They have the required properties.

5.4. We give an alternative proof of Theorem 5.2 in the case where $V,(), Q, n,, \kappa, G$ are as in $\S 1.3$. By $5.2\left(\right.$ a) we can assume that $w=w_{p^{*}}$, where $p_{*}$ is in $\mathcal{P}_{n}$ (if $(1-\kappa) Q=0)$ and in $\mathcal{P}_{n}^{+}$(if $\left.(1-\kappa) Q \neq 0\right)$. Let $V_{*} \in \mathcal{F}$ (if $\left.(1-\kappa) Q=0\right), V_{*} \in \mathcal{F}^{\prime}$ (if $(1-\kappa) Q \neq 0)$ and let $g \in G$ be such that $a_{V_{*}, V_{*}^{\prime}}=w_{p_{*}}$, where $V_{*}^{\prime}=g V_{*}$. It is enough to show that there exists a finite subgroup $\Gamma$ of $G$ such that, if $x \in G$ satisfies $x g x^{-1}=g, x V_{*}=V_{*}$, then $x \in \Gamma$. Consider the basis $\beta$ of $V$ associated in 
3.3 (vi) to $V_{*}, V_{*}^{\prime}, g$. Since $\beta$ is canonically defined (up to multiplication by \pm 1 ) by $V_{*}, V_{*}^{\prime}, g$ we see that the analogous basis associated to $x V_{*}, x V_{*}^{\prime}, x g x^{-1}$ is equal to $x \beta$ (up to multiplication by \pm 1 ). Since $\left(x V_{*}, x V_{*}^{\prime}, x g x^{-1}\right)=\left(V_{*}, V_{*}^{\prime}, g\right)$ we see that $x \beta$ is equal to $\beta$ (up to multiplication by \pm 1 ). Let $\Gamma$ be the set of all elements of $G$ which map each element of $\beta$ to \pm 1 times itself (a finite abelian 2-subgroup of $G)$. We see that $x \in \Gamma$, as required.

5.5. Let $\gamma$ be a conjugacy class of $G$. Let $w \in C_{\min }$. Since $\mathfrak{B}_{w}^{\gamma}$ is a union of $G_{a d}$-orbits in $\mathfrak{B}_{w}$ and each of these orbits has dimension equal to $\operatorname{dim} G_{a d}$ (see Theorem 5.2) we see that if $\mathfrak{B}_{w}^{\gamma} \neq \emptyset$, then $\operatorname{dim} \mathfrak{B}_{w}^{\gamma} \geq \operatorname{dim}\left(G_{a d}\right)$. We say that $\gamma$ is $C$-small if $C \dashv \gamma$ and $\operatorname{dim} \mathfrak{B}_{w}^{\gamma}=\operatorname{dim}\left(G_{a d}\right)$. (The last condition is independent of the choice of $w$ in $C_{\min }$.)

For any Borel subgroup $B$ of $G$ let $\Omega_{B}$ be the $B-B$ double coset of $G$ such that $\left(B, x B x^{-1}\right) \in \mathcal{O}_{w}$ for some/any $x \in \Omega_{B}$. Assume that $\Omega_{B} \cap \gamma \neq \emptyset$. We have the following result:

(a) We have $\operatorname{dim}\left(\Omega_{B} \cap \gamma\right) \geq \operatorname{dim}\left(B / Z_{G}\right)$; moreover, equality holds if and only if $\gamma$ is $C$-small.

Indeed, we have a fibration $\mathfrak{B}_{w}^{\gamma} \rightarrow \mathcal{B},\left(g, B^{\prime}\right) \mapsto B^{\prime}$ whose fibre at $B^{\prime}$ is $\Omega_{B^{\prime}} \cap \gamma$. It follows that $\operatorname{dim}\left(\Omega_{B} \cap \gamma\right)=\operatorname{dim} \mathfrak{B}_{w}^{\gamma}-\operatorname{dim} \mathcal{B}$. It remains to use that $\operatorname{dim}\left(G_{a d}\right)-$ $\operatorname{dim} \mathcal{B}=\operatorname{dim}\left(B / Z_{G}\right)$.

Corollary 5.6. Let $w \in C_{\min }$. Let $g \in G$. The submanifolds $\left\{\left(B, B^{\prime}\right) \in \mathcal{B} \times \mathcal{B}\right.$ : $\left.B^{\prime}=g B g^{-1}\right\}$ and $\mathcal{O}_{w}$ of $\mathcal{B} \times \mathcal{B}$ intersect transversally.

In the case where $g$ is regular semisimple this is proved in [L1, 1.1] (without assumption on $w$ ). As in that proof it is enough to verify the following statement:

(a) if $B \in \mathcal{B}$ and $\left(B, g B g^{-1}\right) \in \mathcal{O}_{w}$, then $(1-\operatorname{Ad}(g))(\mathfrak{g})+\mathfrak{b}=\mathfrak{g}$.

Here $\mathfrak{b}, \mathfrak{g}$ are the Lie algebras of $B, G$. We can assume that $G=G_{a d}$ and that $w$ is good. Let $\mathfrak{g}^{*}$ be the dual space of $\mathfrak{g}$. We give two proofs (the second one applies only for $p=0$ ).

For any subspace $\mathcal{V}$ of $\mathfrak{g}$ let $\mathcal{V}^{\perp}$ be the annihilator of $\mathcal{V}$ in $\mathfrak{g}^{*}$. It is enough to show that $\operatorname{ker}\left((1-\operatorname{Ad}(g)): \mathfrak{g}^{*} \rightarrow \mathfrak{g}^{*}\right) \cap \mathfrak{b}^{\perp}=0$. We argue as in the proof of Theorem 5.2. Let $\xi \in \operatorname{ker}\left((1-\operatorname{Ad}(g)): \mathfrak{g}^{*} \rightarrow \mathfrak{g}^{*}\right) \cap \mathfrak{b}^{\perp}$. Let $d, k, f, \mathbf{s}_{j}^{r}, B_{j}^{r}$ $(r \in[1, m], j \in[0, k d]), m \geq 2$ be as in the proof of Theorem 5.2. Let $\mathfrak{b}_{r}^{j}$ be the Lie algebra of $B_{r}^{j}$. Let $\xi \in \mathfrak{a}$. Since $\operatorname{Ad}(g)^{i} \xi=\xi$ for all $i$ and $\xi \in \mathfrak{b}^{\perp}$ we see that $\xi \in\left(\operatorname{Ad}(g)^{i} \mathfrak{b}\right)^{\perp}$ for $i \in[0, d]$; hence $\xi \in\left(\mathfrak{b}_{i k}^{1}\right)^{\perp}$ for $i \in[0, d]$. Using the definition of $B_{j}^{1}$ for $j \in[0, k d], j \notin k \mathbf{N}$ we see that $\xi \in\left(\mathfrak{b}_{j}^{1}\right)^{\perp}$ for any $j \in[0, k d]$. Using the definitions we see by induction on $r$ that $\xi \in\left(\mathfrak{b}_{j}^{r}\right)^{\perp}$ for any $j \in[0, k d], r \in[1, m]$. In particular we have $\xi \in\left(\mathfrak{b}_{0}^{m}\right)^{\perp} \cap\left(\mathfrak{b}_{f}^{m}\right)^{\perp}$. The last intersection is 0 since $B_{0}^{m}, B_{f}^{m}$ are opposed Borel subgroups. Thus, $\xi=0$, as desired.

In the second proof (with $p=0$ ) let $\mathfrak{n}$ be the Lie algebra of $U_{B}$. We identify $\mathfrak{g}=\mathfrak{g}^{*}$ and $\mathfrak{n}=\mathfrak{b}^{\perp}$ using the Killing form; we see that it is enough to show that $\operatorname{ker}((1-\operatorname{Ad}(g)): \mathfrak{g} \rightarrow \mathfrak{g}) \cap \mathfrak{n}=0$. The last intersection is the Lie algebra of $Z(g) \cap U$. By Theorem 5.2, $Z(g) \cap B$ is a finite group. Hence $Z(g) \cap U$ is a finite subgroup of $U$; hence $Z(g) \cap U=\{1\}$ and the desired result follows.

Corollary 5.7. We preserve the setup of Corollary 5.6.

(i) The variety $\mathcal{B}_{g}^{w}:=\left\{B \in \mathcal{B}:\left(B, g B g^{-1}\right) \in \mathcal{O}_{w}\right\}$ is smooth of pure dimension $\underline{l}(w)$.

(ii) For any $\gamma \in \underline{G}$, the variety $\mathfrak{B}_{w}^{\gamma}$ is smooth of pure dimension $\operatorname{dim} \gamma+\underline{l}(w)$. 
(iii) Assume that $\gamma \in \underline{G}$ and $\mathfrak{B}_{w}^{\gamma} \neq \emptyset$. Then for $g \in \gamma$ we have $\operatorname{dim}\left(Z(g) / Z_{G}\right) \leq$ $\underline{l}(w)$ and $\operatorname{dim} \gamma \geq \operatorname{dim}\left(G_{a d}\right)-\underline{l}(w)$.

(iv) With the assumptions of (iii), $\gamma$ is $C$-small if and only if $\operatorname{dim}\left(Z(g) / Z_{G}\right)=$ $\underline{l}(w)$, that is, if and only if $\operatorname{dim} \gamma=\operatorname{dim}\left(G_{a d}\right)-\underline{l}(w)$.

(We use the convention that the empty variety has dimension $d$ for any $d$.) The variety in (i) may be identified with the intersection in Corollary 5.6 (the submanifolds in Corollary 5.6 have pure dimension $\operatorname{dim} \mathcal{B}, \operatorname{dim} \mathcal{B}+\underline{l}(w)$ and $\mathcal{B} \times \mathcal{B}$ has dimension $2 \operatorname{dim} \mathcal{B}$ ); (i) follows. Now (ii) follows from (i) since $\mathfrak{B}_{w}^{\gamma}$ is fibred over $\gamma$ with fibres as in (i) with $g \in \gamma$. We prove (iii). By Theorem 5.2 every $G_{a d}$-orbit in $\mathfrak{B}_{w}^{\gamma}$ has dimension equal to $\operatorname{dim}\left(G_{a d}\right)$. Hence from (ii) we see that $\operatorname{dim} \gamma+\underline{l}(w) \geq \operatorname{dim}\left(G_{a d}\right)$ and (iii) follows. The proof of (iv) is similar to that of (iii).

5.8. We show:

(a) Assume that $\gamma \in \underline{G}$ is $C$-small. Then the $G_{a d}$-action on $\mathfrak{B}_{w}^{\gamma}$ has finitely many orbits. Also, if $g \in \gamma$, the $Z(g) / Z_{G^{-}}$action on $\mathcal{B}_{g}^{w}$ (by conjugation) has finitely many orbits.

We have $\operatorname{dim} \mathfrak{B}_{w}^{\gamma}=\operatorname{dim}\left(G_{a d}\right)$ (see Corollary 5.7(ii)) and every $G_{a d}$-orbit in $\mathfrak{B}_{w}^{\gamma}$ has dimension equal to $\operatorname{dim}\left(G_{a d}\right)$ (see Theorem 5.2); the first statement of (a) follows. If $g \in \gamma$ then $\operatorname{dim} \mathcal{B}_{g}^{w}=\operatorname{dim}\left(Z(g) / Z_{G}\right)$ (by our assumption and Corollary 5.7(i)). The isotropy groups of the $Z(g) / Z_{G}$-action on $\mathcal{B}_{g}^{w}$ are finite (by Theorem 5.2) hence every $Z(g) / Z_{G}$-orbit in $\mathcal{B}_{g}^{w}$ has dimension $\operatorname{dim}\left(Z(g) / Z_{G}\right)$; the second statement of (a) follows. This proves (a).

In the following result we assume that $p$ is not a bad prime for $G$. We give an alternative characterization of $\Phi(C)$ for $C \in \underline{\mathbf{W}}_{e l}$ which does not involve the partial order of $G$.

(b) There is a unique $C$-small unipotent conjugacy class in $G$, namely $\Phi(C)$. The fact that $\Phi(C)$ is $C$-small follows from 4.4(b) and Corollary 5.7(iv). Assume that $\gamma^{\prime} \in \underline{G}$ is any $C$-small unipotent class. By $\Pi_{C}$ we have $\gamma \subset \bar{\gamma}^{\prime}$. Moreover, we have $\operatorname{dim} \gamma=\operatorname{dim} \gamma^{\prime}$; hence $\gamma=\gamma^{\prime}$. This proves (b). This also proves Theorem 0.7.

5.9. Assume that $\mathbf{k}, F: G \rightarrow G, F: \mathcal{B} \rightarrow \mathcal{B}, G^{F}$ are as in the last paragraph of \$1.2. Assume that $w \in C_{\text {min }}$. Let $X_{w}=\left\{B \in \mathcal{B}:(B, F B) \in \mathcal{O}_{w}\right\}$; see [DL. The finite group $G^{F}$ acts on $X_{w}$ by conjugation. The following result (not used in this paper) is similar to Theorem 5.2.

(a) The isotropy groups of the $G^{F}$-action on $X_{w}$ are abelian of order prime to $p$. The proof is almost identical to that of Theorem 5.2. We can assume that $w$ is good. Let $d, f, s_{1}, s_{2}, \ldots, s_{k}, \mathbf{s}^{1}, \mathbf{s}^{2}, \ldots, \mathbf{s}^{m}(m \geq 2)$ be as in the proof of Theorem 5.2. Let $B \in X_{w}$, let $\mathcal{Z}=\left\{c \in G^{F}: c B c^{-1}=B\right\}=G^{F} \cap B$ and let $c \in \mathcal{Z}$. We define a sequence $B_{0}, B_{1}, \ldots, B_{h d}$ in $\mathcal{B}$ by the following requirements: $B_{i k}=F^{i}(B)$ for $i \in[0, d],\left(B_{i k+j-1}, B_{i k+j}\right) \in \mathcal{O}_{s_{j}}$ for $i \in[0, d-1], j \in[1, k]$. This sequence is uniquely determined. Now conjugation by $c$ preserves each of $B, F B, F^{2} B, \ldots, F^{d} B$; hence (by uniqueness) it automatically preserves each $B_{v}$, $v \in[0, k d]$. Starting with this sequence and using $\mathbf{s}^{1}, \mathbf{s}^{2}, \ldots, \mathbf{s}^{m}$ we define a sequence $B_{*}^{1}, B_{*}^{2}, \ldots, B_{*}^{m}\left(B_{*}^{r}\right.$ is a sequence $\left(B_{0}^{r}, B_{1}^{r}, \ldots, B_{k d}^{r}\right)$ in $\left.\mathcal{B}\right)$ as in the proof of Theorem 5.2. As in Theorem 5.2, any Borel subgroup in the sequence $B_{*}^{r}$ is stable under conjugation by $c$. In particular $B_{0}^{m}, B_{f}^{m}$ contain $c$. From the definitions we see that $\left(B_{0}^{m}, B_{f}^{m}\right) \in \mathcal{O}_{w_{0}}$; that is, $B_{0}^{m}, B_{f}^{m}$ are opposed Borel subgroups. We see 
that $c$ belongs to $B_{0}^{m} \cap B_{f}^{m}$, a maximal torus independent of $c$. Thus $\mathcal{Z}$ is contained in the torus $B_{0}^{m} \cap B_{f}^{m}$. This completes the proof of (a).

Addendum. In 0.4(i), 0.6(i), the assumption that $p$ is not a bad prime for $G$ can now be removed. Indeed, by the arguments in 1.1 it is enough to show that 0.6(i) holds when $p$ is a bad prime for $G$ (which can be assumed to be almost simple). When $G$ is almost simple of exceptional type, 0.6(i) was pointed out in 4.8(a) of [G.Lusztig, Elliptic elements in a Weyl group: a homogeneity property, arxiv:1007.5040]. The proof (based on computer calculations) is the same as in good characteristic, but using the tables for Green functions in bad characteristic from [http://www/math.rwth-aachen. de $/{ }^{\sim}$ Frank. Luebeck/chev/Green/]; the fact that these tables, computed using the algorithm in [L3, Ch. 24], give indeed the Green functions, was proved by M. Geck Ge. When $G$ is almost simple of classical type and $p=2$, then 0.6(i) follows from the results in [George Lusztig and Ting Xue, Elliptic Weyl group elements and unipotent isometries with $p=2$, arxiv:1103.5172]. The same arguments show that 0.6(ii) holds without restriction on $p$ and then so does 0.4(ii); moreover, the map in 0.4(ii) coincides for any $p$ with the map $\Phi$ in 4.5. Now in 5.8(b) and its consequence 0.7, the assumption that $p$ is not a bad prime for $G$ can also be removed in view of the validity of 0.6(i).

\section{REFERENCES}

[Ca] R.W.Carter, Conjugacy classes in the Weyl group, Compositio Math. 25 (1972), 1-59. MR0318337 (47:6884)

[DL] P.Deligne and G.Lusztig, Representations of reductive groups over finite fields, Ann. of Math. (2) 103 (1976), 103-161. MR0393266 (52:14076)

[EG] E.W.Ellers and N.Gordeev, Intersection of conjugacy classes with Bruhat cells in Chevalley groups, Pacific J. Math. 214 (2004), 245-260. MR2042932 (2004m:20091)

[Ge] M.Geck, On the average values of irreducible characters of finite groups of Lie type on geometric conjugacy classes, Doc. Math. 1 (1996), 293-317. MR1418951 (98c:20084)

[Ch] M.Geck, G.Hiss, F.Lübeck, G.Malle and G.Pfeiffer, A system for computing and processing generic character tables for finite groups of Lie type, Weyl groups and Hecke algebras, Appl. Algebra Engrg. Comm. Comput. 7 (1996), 175-210.

[GP] M.Geck and G.Pfeiffer, Characters of finite Coxeter groups and Iwahori-Hecke algebras, Clarendon Press Oxford, 2000. MR.1778802 (2002k:20017)

[Ka] N.Kawanaka, Unipotent elements and characters of finite Chevalley groups, Osaka J. Math. 12 (1975), 523-554. MR0384914 (52:5784)

[KL] D.Kazhdan and G.Lusztig, Fixed point varieties on affine flag manifolds, Israel J. Math. 62 (1988), 129-168. MR947819 (89m:14025)

[L1] G.Lusztig, On the reflection representation of a finite Chevalley group, Representation theory of Lie groups, LMS Lect. Notes Ser. 34, Cambridge Univ. Press, 1979, pp. 325-337.

[L2] G.Lusztig, Characters of reductive groups over a finite field, Ann. Math. Studies 107, Princeton Univ. Press, 1984. MR742472 (86j:20038)

[L3] G.Lusztig, Character sheaves, V, Advances in Math. 61 (1986), 103-155. MR849848 (87m:20118c)

[L4] G.Lusztig, Green functions and character sheaves, Ann. of Math. (2) 131 (1990), 355-408. MR.1043271 (91c:20054)

[L5] G.Lusztig, Hecke algebras with unequal parameters, CRM Monograph Ser.18, Amer. Math. Soc., 2003. MR1974442 (2004k:20011)

[L6] G.Lusztig, On some partitions of a flag manifold, arxiv:0906.1505.

[Lü] F.Lübeck, http://www.math.rwth-aachen.de/ ${ }^{\sim}$ Frank.Luebeck/chev/Green/.

[Mi] K.Mizuno, The conjugate classes of unipotent elements of the Chevalley groups $E_{7}$ and $E_{8}$, Tokyo J. Math. 3 (1980), 391-461. MR605099 (82m:20046)

[Sh] T.Shoji, Character sheaves and almost characters of reductive groups, II, Adv. in Math. 111 (1995), 314-354. MR1318530 (95k:20069) 
[Sp1] N.Spaltenstein, Classes unipotentes et sous-groupes de Borel, Lecture Notes in Math., vol. 946, Springer-Verlag, 1982. MR672610 (84a:14024)

[Sp2] N.Spaltenstein, On the generalized Springer correspondence for exceptional groups, Algebraic groups and related topics, Adv. Stud. Pure Math. 6, North Holland and Kinokuniya, 1985, pp. 317-338. MR803340 (87g:20072b)

[Sp3] N.Spaltenstein, Polynomials over local fields, nilpotent orbits and conjugacy classes in Weyl groups, Astérisque 168 (1988), 191-217. MR1021497 (90k:20069)

[Sp4] N.Spaltenstein, On the Kazhdan-Lusztig map for exceptional Lie algebras, Adv. in Math. 83 (1990), 48-74. MR.1069387 (92b:17010)

[St] R.Steinberg, Regular elements of semisimple algebraic groups, Inst. Hautes Etudes Sci. Publ. Math. 25 (1965), 49-80. MR0180554(31:4788)

Department of Mathematics, Massachusetts institute of Technology, Cambridge, Massachusetts 02139 